\title{
Prediction for minimum bias and the underlying event at LHC energies
}

A. Moraes ${ }^{1}$, C. Buttar ${ }^{2}$, and I. Dawson ${ }^{3}$

${ }^{1}$ Physics Department, Brookhaven National Laboratory, Upton, NY, 11973, USA

2 Department of Physics and Astronomy, University of Glasgow, Kelvin Building, Glasgow, G12 8QQ, UK

3 Department of Physics and Astronomy, University of Sheffield The Hicks Building, Sheffield, S3 7RH, UK

Re-submitted: 23rd January 2007

\begin{abstract}
In this report we investigate the models employed by PYTHIA and PHOJET Monte Carlo event generators used to describe soft interactions in hadronhadron collisions. The aim of this study is to predict the important properties of minimum bias and the underlying event at the LHC energy scale. Focusing on a wide range of measurements dominated by soft interactions in proton-proton and proton-anti-proton collisions, one of the aims of this study is to check the consistency of these models when compared to data and evaluate the accuracy of their descriptions of low- $\mathrm{p}_{t}$ processes. Based on comparisons to a wide range of minimum bias and underlying event data we present a tuning for PYTHIA6.214 and compare it to other PYTHIA tunings. Our proposed tuning for PYTHIA6.214 has been used for the ATLAS Data Challenge productions.
\end{abstract}

PACS. 12.40.-y Models of strong interactions - 13.85.Hd High-energy interactions of hadrons

e-mail: amoraes@bnl.gov

\section{Introduction}

At the Large Hadron Collider (LHC) [1], essentially all physics processes will arise from quark and gluon interactions. Probed at high-energy, protons, or indeed any other hadron, look like clusters of confined "partons", i.e. quarks (anti-quarks) and gluons. High-energy proton collisions at the LHC can therefore be described in terms of parton interactions [2]. However, our ability to describe parton scatterings through quantum chromodynamics (QCD) depends on the amount of transverse momenta with respect to the collision axis $\left(\mathrm{p}_{t}\right)$ involved in a given scattering [3].

QCD has been very successful in describing quark, anti-quark and gluon scatterings involving large amounts of transverse momenta known as "hard" interactions. However high-energy pp and $\mathrm{p} \overline{\mathrm{p}}$ collisions are dominated by soft partonic collisions, so-called minimum bias events. Soft partonic interactions also occur in the remains of hard scattering events not associated with the hard process and this is important for many physics analyses such as $\mathrm{W}$ boson and top-quark mass measurements [4], Higgs VBF [5] and SUSY searches.

As in previous hadron colliders, soft interactions will also be the dominant processes in proton-proton collisions at the LHC. Hence, most of the particles produced at the LHC will originate from soft interactions. This is particularly relevant for predictions of background levels associated to many physics processes and also for understanding the complex nature of the radiation environment in which the LHC's detector systems will operate [6].

QCD breaks in the region of soft interactions due to two effects. At $\Lambda_{\mathrm{QCD}} \sim$ few hundred $\mathrm{MeV}, \alpha_{s} \sim 1$ and perturbative QCD breaks down. At momentum transfer of $\sim$ few $\mathrm{GeV}, \sigma_{\mathrm{QCD}}$ for a $2 \rightarrow 2$ parton scattering will exceed the pp or $\mathrm{p} \overline{\mathrm{p}}$ cross-section. One method of solving this has been to introduce multiparton interactions for which there is an increasing number of supporting experimental evidence [7-9].

Current models of high-energy hadron collisions will typically combine perturbative QCD to explain parton interactions where it is applicable (high- $\mathrm{p}_{t}$ scatterings), with an alternative phenomenological approach to describe soft processes. Examples of these are the Dual Parton Model (DPM) [10] and modified versions of QCD in which the divergences presented by the running coupling constant are phenomenologically corrected to reproduce experimental observations [11].

In this paper we assume that minimum bias events and the underlying event, or many aspects of them, can be described by the same physical model as has been done, for example, in PYTHIA [12]. Minimum bias data have been collected in the energy range $200 \mathrm{GeV} \rightarrow 1.8 \mathrm{TeV}$ [13-19]. This allows many aspects of the model to be tuned but especially allows the energy dependence to be investigated. The underlying event data from CDF measurements at $1.8 \mathrm{TeV}[20]$ have been used to tune the model description of the underlying event. This method, which combines minimum bias and underlying event data-sets, has meant we have been unable to investigate HERWIG with the JIMMY package [21] as this does not describe minimum bias events since it operates above a $\mathrm{p}_{t}$-cut-off and only describes the underlying event.

In sect. 2 we investigate two Monte Carlo (MC) event generators, PYTHIA [12] and PHOJET [22], focusing on their models for soft interactions in hadron-hadron collisions. Aiming to check the consistency of these models, in 
sects. 3 and 4 we compare their predictions to wide range of data which demand of them an efficient description of low- $\mathrm{p}_{t}$ processes. Comparisons to minimum bias data are shown in sect. 3 and to the underlying event measured by CDF, in sect. 4. A proposed tuning for PYTHIA6.214 is presented in sect. 5 , where we also compare our tuning predictions to those generated with alternative PYTHIA tunings. Predictions for levels of particle production and event activity at the LHC for interactions dominated by soft processes such as minimum bias interactions and the underlying event associated to jet production are discussed in sect. 6. Finally, in sect. 7 we present our conclusions.

\section{$2 \mathrm{MC}$ event generators}

\subsection{PYTHIA model for hadron collisions}

In the PYTHIA model the total rate of parton interactions, $N_{\text {parton-parton, as a function of the transverse mo- }}$ mentum scale $\mathrm{p}_{t}$, is assumed to be given by perturbative QCD. At reasonably large $\mathrm{p}_{t}$ values $\left(\mathrm{p}_{t} \gtrsim 2 \mathrm{GeV}\right)$ parton scatterings can be correctly described by the standard perturbative QCD, but to extend the parton-parton scattering framework to the low- $\mathrm{p}_{t}$ region a regularisation to correct the divergence in the cross-section is introduced.

The starting point of this model is provided by the perturbative QCD cross-section for parton-parton interactions which is given by:

$$
\begin{array}{r}
\sigma=\sum_{i, j, k} \int d x_{1} \int d x_{2} \int d \hat{t} \hat{\sigma}_{i, j}^{k}(\hat{s}, \hat{t}, \hat{u}) \\
\times f_{A}^{i}\left(x_{1}, Q^{2}\right) f_{B}^{j}\left(x_{2}, Q^{2}\right),
\end{array}
$$

where $\hat{\sigma}_{i, j}^{k}$ is the hard-scattering cross-section for the kth subprocess possible between incoming partons $i$ and $j$ and $\hat{s}, \hat{t}$ and $\hat{u}$ are the Mandelstam variables. For massless partons, the three Mandelstam variables are related by

$$
\hat{s}+\hat{t}+\hat{u}=0
$$

and

$$
\hat{s}=x_{1} x_{2} s .
$$

The parton distribution functions $f_{A}^{i}\left(x_{1}, Q^{2}\right)$ $\left(f_{B}^{j}\left(x_{2}, Q^{2}\right)\right)$ give the probability to find a parton $i(j)$ with a fraction $x_{1}\left(x_{2}\right)$ of the beam energy inside the incoming hadron A (B), if it is probed at a scale $\mathrm{Q}^{2}$. Finally, the $\mathrm{Q}^{2}$ scale is set to

$$
Q^{2}=p_{t}^{2}=\frac{\hat{t} \hat{u}}{\hat{s}}
$$

The differential cross-section as a function of $\mathrm{p}_{t}^{2}$ describing a $2 \rightarrow 2$ parton scattering is given by

$$
\begin{array}{r}
\frac{d \sigma}{d p_{t}^{2}}=\sum_{i, j, k} \int d x_{1} \int d x_{2} \int d \hat{t} f_{A}^{i}\left(x_{1}, Q^{2}\right) f_{B}^{j}\left(x_{2}, Q^{2}\right) \\
\times \frac{d \hat{\sigma}_{i j}^{k}}{d \hat{t}} \delta\left(p_{t}^{2}-\frac{\hat{t} \hat{u}}{\hat{s}}\right),
\end{array}
$$

and the interaction cross-section above any chosen $\mathrm{p}_{t_{\text {min }}}$ limit is thus written as

$$
\sigma_{i n t}\left(p_{t_{m i n}}\right)=\int_{p_{t_{\text {min }}}^{2}}^{s / 4} \frac{d \sigma}{d p_{t}{ }^{\prime 2}} d p_{t}{ }^{\prime 2} .
$$

This introduces two problems that must be solved. The first one is observed at $\mathrm{p}_{t} \sim 2 \mathrm{GeV}$. Since $\Lambda_{Q C D} \sim$ $0.2 \mathrm{GeV}$, perturbative QCD can still be applied at this $\mathrm{p}_{t}$ region. However, the interaction cross-section exceeds the total cross-section. The second problem happens as lower $\mathrm{p}_{t}$ values are used. Defining the momentum fraction $\mathrm{x}$ as the approximated value $\mathrm{x} \sim\left|\mathrm{p}_{t}\right| / \sqrt{s}$, low- $\mathrm{p}_{t}$ parton scatterings probe the parton densities at small-x values. As $\mathrm{p}_{t} \rightarrow 0$, the small-x region where parton distribution functions rise quite steeply, give the major contribution to the integral in eq.(6) and the differential cross-section diverges roughly like $d \mathrm{p}_{t}^{2} / \mathrm{p}_{t}^{4}[11]$.

The first problem is solved by introducing the concept of multiple parton interactions [11]. At high-energy, each of the incoming hadrons may be viewed as a beam of partons hence there is a possibility of having several partonparton interactions when the hadrons collide. Thus, events with $\sigma_{\text {int }}\left(\mathrm{p}_{t_{\text {min }}}\right)>\sigma_{\text {tot }}$ are interpreted as having more than one parton-parton interaction taking place in the event. According to this model, the average number of parton scatterings in the event is defined as

$$
N_{\text {parton-parton }}=\frac{\sigma_{\text {int }}\left(p_{t_{\text {min }}}\right)}{\sigma_{n d}}
$$

where $\sigma_{n d}$ is the non-diffractive inelastic interaction crosssection [11].

The multiple parton scatterings in hadron collisions have been observed by experiments such as AFS [7], UA2 [8] and most recently has been directly measured by the CDF Collaboration [9].

The second problem, which remains after multiparton interactions are introduced, is related to the divergence observed as $\mathrm{p}_{t} \rightarrow 0$, is solved by introducing a cut-off parameter $\mathrm{p}_{t_{\text {min }}}$ given by [11]

$$
\mathrm{p}_{t_{\text {min }}}(s)=(1.9 \mathrm{GeV})\left(\frac{s}{1 \mathrm{TeV}^{2}}\right)^{0.08} .
$$

The cut-off parameter $\mathrm{p}_{t_{\text {min }}}$ can be interpreted as the inverse of some colour screening length, $d$, in the hadron $[11,23]$ (see fig. 1). Equation 8, shows $\mathrm{p}_{t_{\min }}$ with the default energy dependence in the PYTHIA versions released after the version 6.200. The energy dependence shown in eq.(8), is taken to vary with the power 0.08 following the same energy dependence as observed in the total crosssection [24].

There are two strategies, or scenarios, proposed by the Lund model to introduce the cut-off parameter defined by eq. (8).

In the first one, labelled "simple" scenario, an effective cut-off is established at $\mathrm{p}_{t_{\text {min }}}$, which means that $d \sigma / d p_{t}^{2}=$ 0 for $\mathrm{p}_{t}<\mathrm{p}_{t_{\text {min }}}$. In other words, a sharp cut-off at $\mathrm{p}_{t_{\text {min }}}$ is equivalent to establishing a maximum impact parameter, $\mathrm{b}_{\max }$, above which there is no interaction. The sharp 


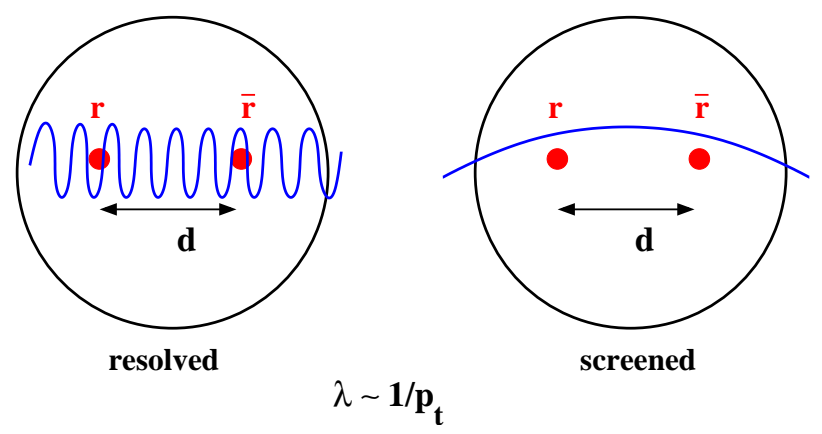

Fig. 1. Illustration of resolved $(\lambda<\mathrm{d})$ and screened $(\lambda>\mathrm{d})$ colour charges in a hadron.

cut-off applied to the probability of parton-parton interactions may also be interpreted as a consequence of the parton confinement. In this framework, valence quarks from incoming hadrons carrying a transverse component of momentum $\mathrm{p}_{t}<\mathrm{p}_{t_{\min }}$ would not be able to overcome the partonic binding and interact inelastically. For interactions with $\mathrm{p}_{t}<\mathrm{p}_{t_{\min }}$ there will be an exchange of a very soft gluon between the two colliding hadrons which does not affect the momentum distribution of partons, but transforms hadrons into colour-octet objects (two-string picture) [11].

Since each incoming hadron is a composite object, consisting of many partons, there should exist the possibility of several parton pairs interacting when two hadrons collide. The model assumes that different pairwise interactions take place essentially independent of each other, and that therefore the number of interactions in an event is given by a Poissonian distribution. This is the strategy of the simple scenario [11].

Considering that hadrons are not only composite but also extended objects, partonic interaction probabilities are likely to vary for each reaction. In this approach, called the 'complex' scenario, the probability associated with each interacting parton depends on the assumed matter distribution inside the colliding hadrons. In the 'complex' scenario an impact parameter dependent approach is therefore introduced [11]. A small b value $\left(\mathrm{p}_{t}>>\mathrm{p}_{t_{\text {min }}}\right)$ corresponds to a large overlap between the two colliding hadrons and hence an enhanced probability of multiple interactions. On the other hand, a large $\mathrm{b}\left(\mathrm{p}_{t}<\mathrm{p}_{t_{\text {min }}}\right)$ means a large probability that no parton-parton interaction will take place in the event. This causes the so-called pedestal effect seen by UA1 [11] and also affects charged multiplicity distributions.

In this scenario, the divergences at $\mathrm{p}_{t} \rightarrow 0$ are corrected by multiplying the matrix elements by a factor $\mathrm{p}_{t}^{4} /\left(\mathrm{p}_{t}^{2}+\mathrm{p}_{t_{\text {min }}}^{2}\right)^{2}$ and replacing the argument $\mathrm{p}_{t}^{2}$ in $\alpha_{s}$ by $\left(\mathrm{p}_{t}^{2}+\mathrm{p}_{t_{m i n}}^{2}\right)$. This action removes divergent terms proportional to $1 / \mathrm{p}_{t}^{4}$, provides a continuous $\mathrm{p}_{t}$ spectrum for calculated interaction properties, and reduces to standard perturbative QCD for $\mathrm{p}_{t}>>\mathrm{p}_{t_{\text {min }}}$ [11].

The parameters defining $\mathrm{p}_{t_{\min }}$ as shown in eq.(8) are displayed in table 1 . The factor $1.9 \mathrm{GeV}$ is defined in the simple scenario by $\operatorname{PARP}(81)$ and by $\operatorname{PARP}(82)$ in the

\begin{tabular}{|c|c|}
\hline \multicolumn{2}{|c|}{$\mathbf{p}_{t_{\text {min }}}$ parameters } \\
\hline $\operatorname{PARP}(81)=1.9$ & simple scenario \\
$\operatorname{PARP}(82)=1.9$ & complex scenario \\
$\operatorname{PARP}(89)=1 \mathrm{TeV}$ & energy scale \\
$\operatorname{PARP}(90)=0.16$ & power which regulates \\
& $\mathrm{p}_{t_{\text {min }}}$ 's energy dependence \\
\hline
\end{tabular}

Table 1. Default parameters for $\mathrm{p}_{t_{\text {min }}}$ as defined in PYTHIA 6.214 .

complex scenario. The energy scale of $1 \mathrm{TeV}$ is defined by $\operatorname{PARP}(89)$ and is included in eq.(8) to be a convenient tuning parameter rather than a parameter with physical meaning. PARP $(90)$ gives the power with which $\mathrm{p}_{t_{\min }}$ varies with the centre of mass energy, $\sqrt{s}$. The default option is set as $\operatorname{PARP}(90)=0.16$ [12].

In both scenarios, all the scatterings that occur in an event must be correlated somehow by momentum and flavour conservation for the partons from each incoming hadron, as well as by various quantum-mechanical effects. Finally, after the parton interaction cross-sections are estimated by the model, the resulting partons are fragmented into colourless hadrons. This introduces a correlation between the final-state hadrons and the number of multiple interactions.

\subsection{PHOJET}

The physics model used in the MC event generator PHOJET combines the ideas of the Dual Parton Model (DPM) [10] with perturbative QCD [3] to give an almost complete picture of high-energy hadron collisions [22].

PHOJET is formulated as a two-component model containing contributions from both soft and hard interactions. The DPM is used to describe the dominant soft processes and perturbative QCD is applied to generate hard interactions [22].

Combining non-perturbative topological expansions of QCD with generally accepted theoretical principles like duality, unitarity, Regge behaviour and the parton structure of hadrons, the DPM provides a complete phenomenological description of soft processes in high-energy hadron collisions [10].

The mechanism of Pomeron exchange is at the heart of the DPM [10]. According to the DPM, the leading contribution to multiparticle production in high-energy hadron-hadron collisions stems from the exchange of a single Pomeron between the colliding hadrons. Secondary Pomeron exchanges account for the remaining activity in the event.

Each exchanged Pomeron gives rise to two colour-neutral chains stretching between quarks and diquarks, for baryons, or quarks and anti-quarks for mesons or pairs 


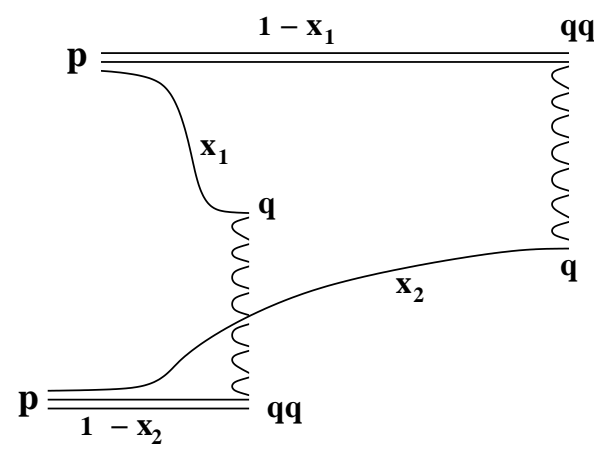

(a)

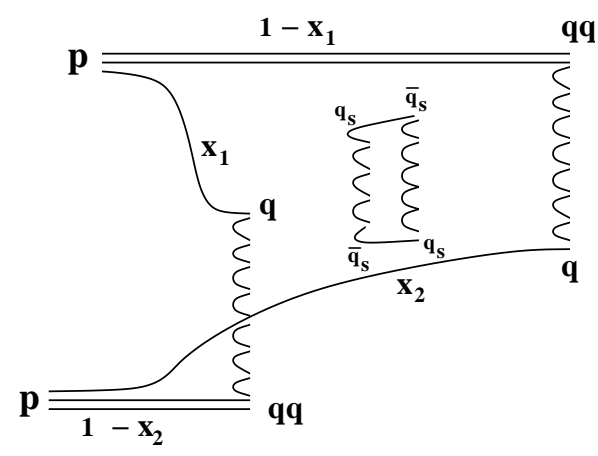

(b)

Fig. 2. (a) Dominant two-chain diagram corresponding to a single Pomeron exchange and (b) leading two-chain contribution with a secondary pair of chains corresponding to the exchange of two Pomerons in high-energy pp collision.

of sea quarks and anti-quarks polarised from the vacuum. In the case of the leading Pomeron exchange, these chains stretch between valence quarks (or anti-quarks) whereas for the other exchanges the chains will end on valence and sea quarks from the initial hadrons.

As an example, the dominant two-chain diagram describing multiparticle production in high-energy protonproton (pp) collisions is shown in figure 2(a) and the leading two-chain contribution with a secondary pair of chains corresponding to the exchange of two Pomerons in figure $2(\mathrm{~b})$.

The model employed by PHOJET is based on the calculation of scattering amplitudes, taking into account the unitarization principle. Comparisons between the calculated results for cross-sections and the available data have been made to determine the unknown model parameters (couplings, Pomeron intercepts and slope parameters), which are needed to generate multiparticle final states produced in inelastic interactions [22].

\subsubsection{The two-component model}

In PHOJET, the division of inelastic hadronic interactions into soft and hard processes is similar to that used in PYTHIA and a $\mathrm{p}_{t}^{\text {cut-off }}$ separating soft and hard interactions is also introduced in PHOJET.

The total cross-section, $\sigma_{t o t}$, is obtained by adding the soft, $\sigma_{\text {soft }}$, and the hard, $\sigma_{\text {hard }}$, cross-section with the cor- rections imposed by unitarity. The soft and hard crosssections vary for different values of $\mathrm{p}_{t}^{\text {cut-off }}$.

Within the DPM, Pomeron exchanges dominate the soft processes in high energy hadron-hadron interactions. The Pomeron exchange cross-section for pure soft interactions can be parametrised as [25]

$$
\sigma_{\text {soft }}=37.8 s^{0.076}(\mathrm{mb}) .
$$

As shown in eq.(9), the soft cross-section increases with the centre-of-mass energy as a power of s. The total crosssection has been observed to grow as $(\ln \mathrm{s})^{2}[25,26]$ which means that as $\mathrm{s} \rightarrow \infty, \sigma_{\text {soft }}$ becomes bigger than the $\sigma_{\text {tot }}$, violating the unitarity bound. The mechanism used to preserve unitarity consists of including multiple Pomeron exchange as an additional component in the hadron interaction, a model which agrees with the experimental evidence [10].

The hard cross-section is calculated with a lower transverse momentum cut greater than $2 \mathrm{GeV}$ [25]. As for the soft cross-section, the hard cross-section increases with the centre-of-mass energy $\sqrt{s}$ approximately as a power of $\mathrm{s}$ [25]. The expression for hard scattering cross-section is given by

$$
\begin{array}{r}
\sigma_{h}=\sum_{i, j \rightarrow k, l} \int d x_{1} \int d x_{2} \int d \hat{t} x_{1} f_{i}\left(x_{1}, Q^{2}\right) x_{2} f_{j}\left(x_{2}, Q^{2}\right) \\
\times \frac{1}{x_{1} x_{2}} \pi M^{2} \frac{\alpha_{s}^{2}\left(Q^{2}\right)}{\hat{s}^{2}},
\end{array}
$$

where $f_{i, j}\left(x, Q^{2}\right)$ are the parton distributions, $M=M_{i, j \rightarrow k, l}$ is the matrix element for the hard partonparton scattering $i, j \rightarrow k, l$, and $\alpha_{s}\left(\mathrm{Q}^{2}\right)$ is the strong coupling constant at the hard scale $\mathrm{Q}^{2}[25]$.

For increasingly higher $\sqrt{s}$, events with $\sigma_{\text {hard }}>\sigma_{\text {tot }}$ will appear, and similarly to the mechanism of multiple soft interactions which explains interactions with $\sigma_{\text {soft }}>$ $\sigma_{t o t}$, a mechanism of multiple hard parton scatterings must be adopted to preserve unitarity. Therefore PHOJET allows the possibility of having events with multiple soft interactions (multiple Pomeron exchanges) and multiple hard parton scatterings.

In addition to soft and hard interactions as described above, PHOJET also allows the possibility of having initial and final state parton showers. These parton showers are generated in leading-log approximation [22].

The fragmentation of soft-chains or hard scattered partons is done by the Lund model $[12,22]$, i.e. PHOJET uses the same fragmentation model adopted by PYTHIA [27].

The soft, $\sigma_{\text {soft }}$, and hard, $\sigma_{\text {hard }}$, cross-sections are inclusive cross-sections and the average multiplicities of soft and hard scatterings in an inelastic event are

$$
\begin{aligned}
& \left\langle n_{s}\right\rangle=\frac{\sigma_{s}}{\sigma_{\text {inel }}}, \\
& \left\langle n_{h}\right\rangle=\frac{\sigma_{h}}{\sigma_{\text {inel }}},
\end{aligned}
$$


respectively. The hard scatterings are mostly independent of each other, being related only by the sharing of energy and momentum of the incoming protons. These multiplicities increase with the colliding centre-of-mass energy. For pp collisions at $\sqrt{s}=14 \mathrm{TeV}$ a considerable part of interactions is expected to have more than one hard or soft scattering.

\subsection{PYTHIA vs. PHOJET}

The starting point for the event generation in PYTHIA is the description of possible hard interactions in $\mathrm{e}^{+} \mathrm{e}^{-}$, pp, p $\bar{p}$ or ep colliders. However, as shown above, it also combines sophisticated models dealing with soft hadronic interactions [12]. PHOJET, on the other hand, initialises the event generation by describing the soft component of hadron-hadron, photon-hadron or photon-photon interactions at high energies. The hard component, calculated by perturbative QCD at the partonic level, is then introduced to complete the event simulation [22]. Due to the different underlying theoretical models used in the physics simulation by these two event generators, PYTHIA presents the user with hundreds of settings that can be adjusted in order to better reproduce the data while PHOJET is relatively more tightly restricted.

Throughout the next sections, we will present analysis based on both MC event generators, however the emphasis will be in adapting the PYTHIA model to the data while using PHOJET as a reference to evaluate uncertainties which appear when these event generators, especially their models for soft interactions, are extrapolated to the LHC energies. Note that parameters not mentioned in the text are kept as default in comparisons shown throughout this article. The PHOJET parameters used in our simulations are the ones found in Ref. [22]. The physical meaning of the relevant PYTHIA parameters to be investigated is summarized in Appendix A. The program versions used in this study are PYTHIA version 6.214 (PYTHIA6.214) and PHOJET version 1.12 (PHOJET1.12).

\section{Cross-section predictions}

The total collision cross-section for hadron-hadron scatterings, $\sigma_{\text {tot }}$, can be divided into elastic $\left(\sigma_{\text {elas }}\right)$ and inelastic $\left(\sigma_{\text {inel }}\right)$ processes. The inelastic scattering crosssection is also usually sub-divided into single $\left(\sigma_{s d}\right)$ and double $\left(\sigma_{d d}\right)$ diffractive and non-diffractive inelastic crosssections $\left(\sigma_{n d}\right)[28]$. The total cross-section, $\sigma_{t o t}$, can thus be written as

$$
\sigma_{\text {tot }}(s)=\sigma_{\text {elas }}(s)+\sigma_{s d}(s)+\sigma_{d d}(s)+\sigma_{n d}(s),
$$

where $\mathrm{s}$ is the square of the total centre of mass energy.

As shown in eqs.(7) and (11), both models rely on components of the hadronic cross-section $\left(\sigma_{n d}\right.$ and $\sigma_{\text {inel }}$ used by PYTHIA and PHOJET, respectively) to estimate the rate of multi-parton scattering or multi-Pomeron exchanges. In this section we investigate cross-section predictions for $\mathrm{pp}$ and $\mathrm{p} \overline{\mathrm{p}}$ collisions generated by PYTHIA6.214 and PHOJET 1.12.

Figure 3 shows a schematic view of elastic (a), singlediffractive (b), double-diffractive (c) and non-diffractive inelastic hadron interactions (d) in the $\eta-\phi$ phase space, the angle $\phi$ being the azimuthal scattering direction and $\eta$ the pseudorapidity.

As schematically shown in fig.3(a), the hadron separation in pseudorapidity is maximum for elastic scatterings. Single and double diffractive events, shown in figs. $3(\mathrm{~b})$ and (c) respectively, display clear separation, or gaps, between the systems travelling in the forward and backward regions. However, for particles produced in a nondiffractive event, as displayed in 3(d), gaps which naturally occur between two systems moving in opposite directions (forward and backward) are filled by particles produced in the central region.

Based on a parametrisation derived from the Pomeron exchange model [24], PYTHIA generates the total pp and $\mathrm{p} \overline{\mathrm{p}}$ cross-section (mb) using the following expressions:

$$
\begin{aligned}
& \sigma_{\text {tot }}^{p p}(s)=21.7 s^{0.0808}+56.1 s^{-0.4525} \\
& \sigma_{t o t}^{p \bar{p}}(s)=21.7 s^{0.0808}+98.4 s^{-0.4525}
\end{aligned}
$$

where the first term on the right hand side of both eqs.(13) and (14) is the Pomeron contribution and the second one a Reggeon exchange [12].

In PHOJET, the total pp and $\mathrm{p} \overline{\mathrm{p}}$ cross-section are generated through the optical theorem and are corrected for high energies using the unitarity principle [22]. The differential elastic cross-section is related to the scattering amplitude $\mathrm{A}(\mathrm{s}, \mathrm{t})$ by

$$
\frac{d \sigma_{\text {elas }}}{d t}=\frac{1}{16 \pi s^{2}}|A(s, t)|^{2}
$$

and through the optical theorem relation

$$
\sigma_{\text {elas }}=\frac{\sigma_{\text {tot }}^{2}}{16 \pi B_{\text {elas }}}
$$

the total cross-section $\sigma_{t o t}$ is estimated, with $B_{\text {elas }}$ being the elastic slope and $\sigma_{\text {elas }}$ the elastic cross-section.

The resulting total $\mathrm{pp}$ and $\mathrm{p} \overline{\mathrm{p}}$ cross-sections are proportional to $(\ln \mathrm{s})^{2}$ [25] and will not violate the FroissartMartin bound which postulates that $\sigma_{t o t}<\mathrm{A} \ln ^{2} \mathrm{~s}$, where the constant $\mathrm{A} \sim 60 \mathrm{mb}[26]$.

As can be seen in fig.4 the total cross-sections for pp and $\mathrm{p} \overline{\mathrm{p}}$ are very similar to each other for $\sqrt{s}>10 \mathrm{GeV}$. A plausible explanation can be derived from the Pomerom exchange model: as shown in eqs.(13) and (14), for $\sqrt{s} \rightarrow$ $\infty$ the Pomerom term, which is the same for both pp and $\mathrm{p} \overline{\mathrm{p}}$ total cross-sections, dominates, leading to a high similarity between the two cross-sections at high energies. Figure 4 also shows good agreement between the predictions of the generators and the experimental data for energies below $1 \mathrm{TeV}$. For energies higher than $\sim 800 \mathrm{GeV}$ the predictions start to diverge at the level of $\sim 15 \%$. The total 

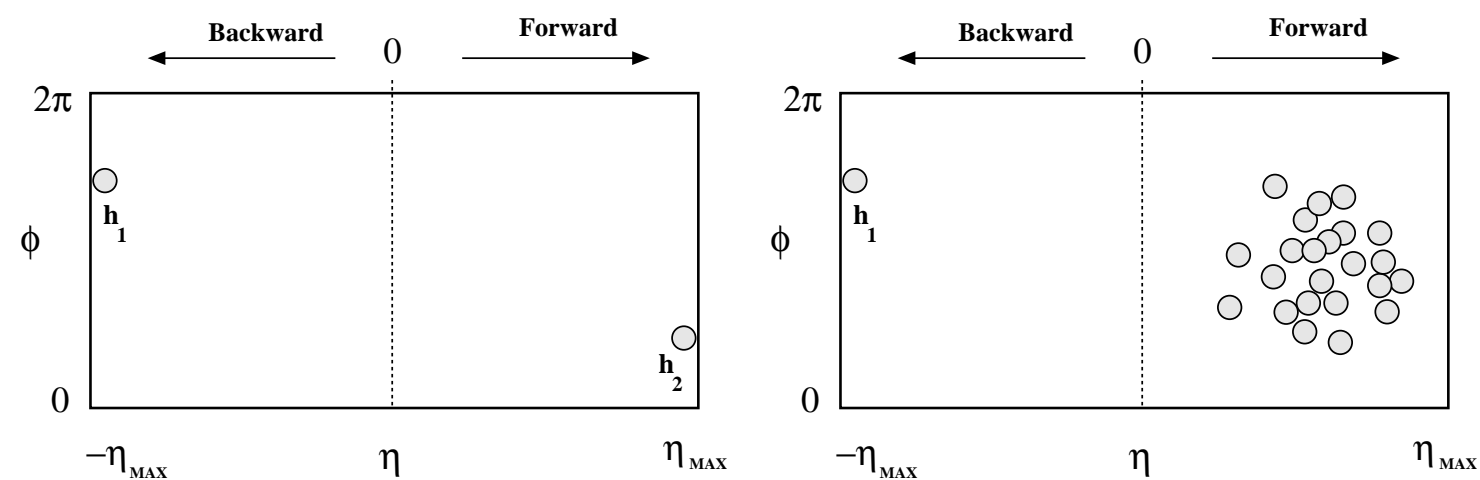

(a)

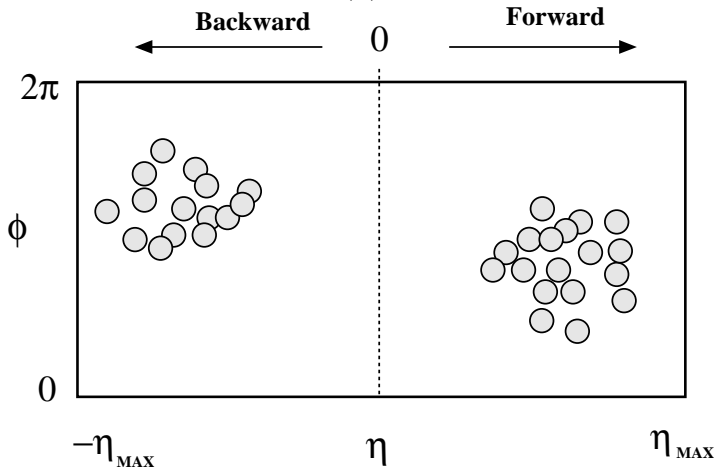

(c)

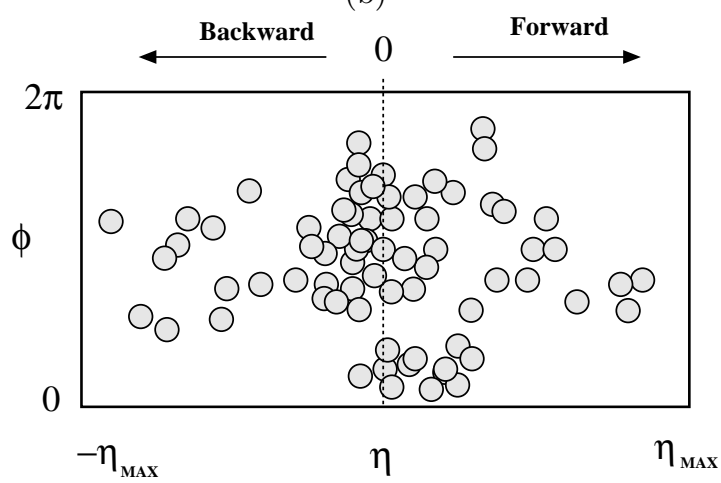

(d)

Fig. 3. Schematic view of (a) elastic, (b) single-diffractive, (c) double-diffractive and (d) non-diffractive hadron interactions in the $\eta-\phi$ space.

cross-section predictions for pp collisions at $14 \mathrm{TeV}$ are $\sigma_{\text {tot }}=101.5 \mathrm{mb}$ and $\sigma_{t o t}=119.1 \mathrm{mb}$ for PYTHIA6.214 and PHOJET1.12, respectively. Recent theoretical studies predict $\sigma_{\text {tot }}=106.3 \pm 5.1_{\text {syst. }} \pm 2.4_{\text {stat. }}$ mb for pp collisions at the $\mathrm{LHC}[29]$.

Once PYTHIA calculates the total cross-section, the same expression from the optical theorem used by PHOJET, given in eq.(16), (with different parametrizations for the elastic slope $B_{\text {elas }}$ in each program), is used to estimate the elastic cross-section $[12,22]$. In both event generators, the inelastic part of the interaction is obtained by subtracting the elastic from the total cross-section.

The inelastic and elastic contributions to the total pp cross-section generated by PYTHIA6.214 and PHOJET1.12 are displayed in fig. 5(a). As expected [28], inelastic events are the major contributors to the total crosssection. The pattern of the distributions, $\sigma_{t o t}$ and $\sigma_{i n e l}$, is the same, with the divergences between the predicted cross-sections starting at roughly the same energy, but being less pronounced in the $\sigma_{\text {inel }}$ distribution. The elastic cross-sections on the other hand, present a considerable divergence for $\sqrt{s}>1 \mathrm{TeV}$. This is mostly due to the differences in $\sigma_{t o t}$ which are magnified in $\sigma_{\text {elas }}$ as indicated by eq.(16). At the LHC energy, PYTHIA6.214 predicts $\sigma_{\text {elas }}=22.5 \mathrm{mb}$ while PHOJET1.12 estimates $\sigma_{\text {elas }}=34.5 \mathrm{mb}, 53 \%$ greater than the former.

\subsection{Minimum bias interactions}

It is not unusual to find slightly different definitions for minimum bias events in the literature. Historically, from the days of CERN-ISR [13], CERN-SPS UA5 [14] up to the more recent Tevatron experiments CDF [15] and E735 [16], measurements of minimum bias events are a close approximation of non-single diffractive inelastic (NSD) interactions, i.e. $\sigma_{n s d}=\sigma_{\text {tot }}-\sigma_{\text {elas }}-\sigma_{s d}$. This interpretation is based on the triggering system used in the experiments mentioned above which favoured the detection of NSD events. On the other hand, with the re-ignited interest for theoretical models that could satisfactorily describe soft interactions and diffractive processes, some groups have identified 'minimum bias events' as non-diffractive inelastic interactions [28].

In practical terms, the experimental choice for nonsingle diffractive inelastic interactions as minimum bias events does not differ considerably from the more theoretical non-diffractive choice, since their cross-sections would hardly differ by more than $\sim 15 \%$ at the current collider energies. Throughout this article, we will associate minimum bias events with NSD inelastic interactions, following the experimental trend. As shown in table 2, in the language of the MC event generators used in this work, subprocesses 94 and 95 are switched on in PYTHIA6.214, and processes $\operatorname{IPRON}(1,1), \operatorname{IPRON}(4,1)$ and $\operatorname{IPRON}(7,1)$ in PHOJET1.12. Note that in PHOJET we are also including central diffraction $(\operatorname{IPRON}(4,1))$ as part of the 


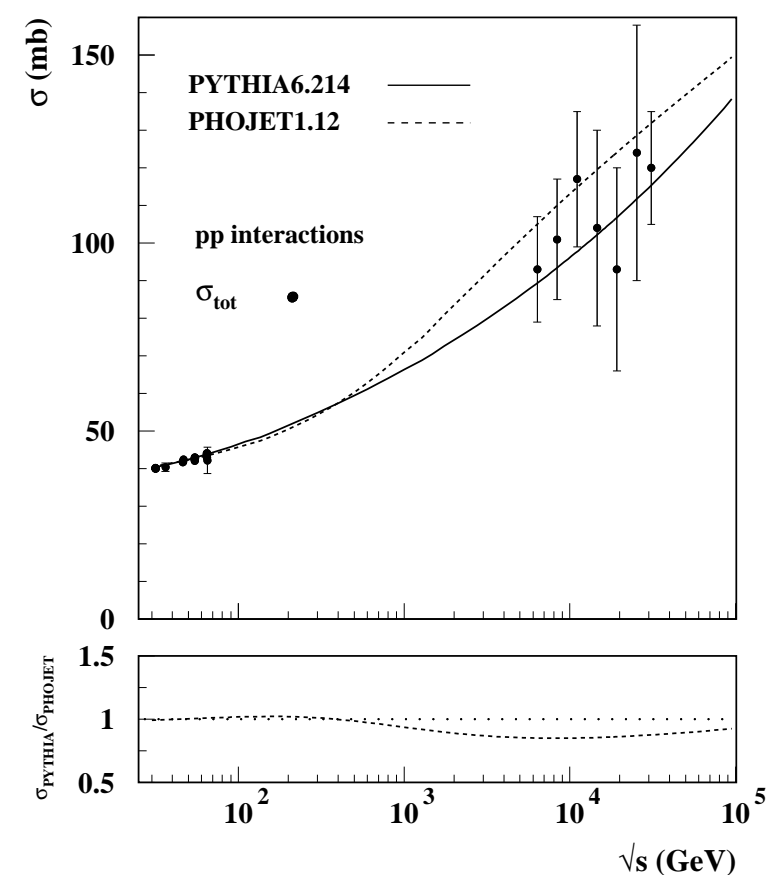

(a)

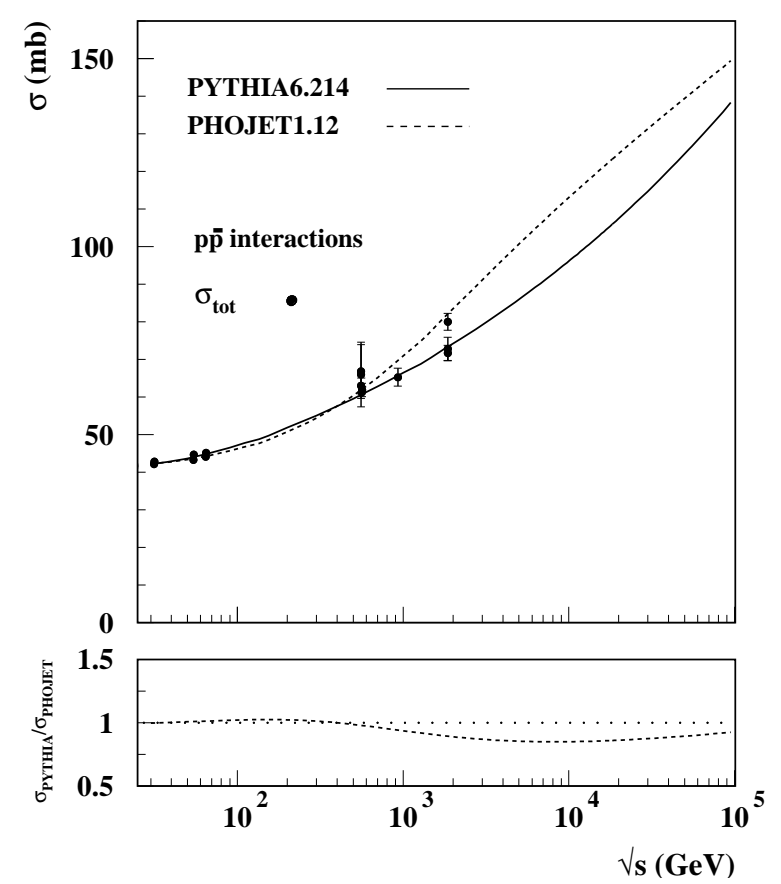

(b)

Fig. 4. PYTHIA6.214 and PHOJET1.12 total cross-section for pp (a) and $\mathrm{p} \overline{\mathrm{p}}(\mathrm{b})$ interactions. Data points taken from Ref. [30].

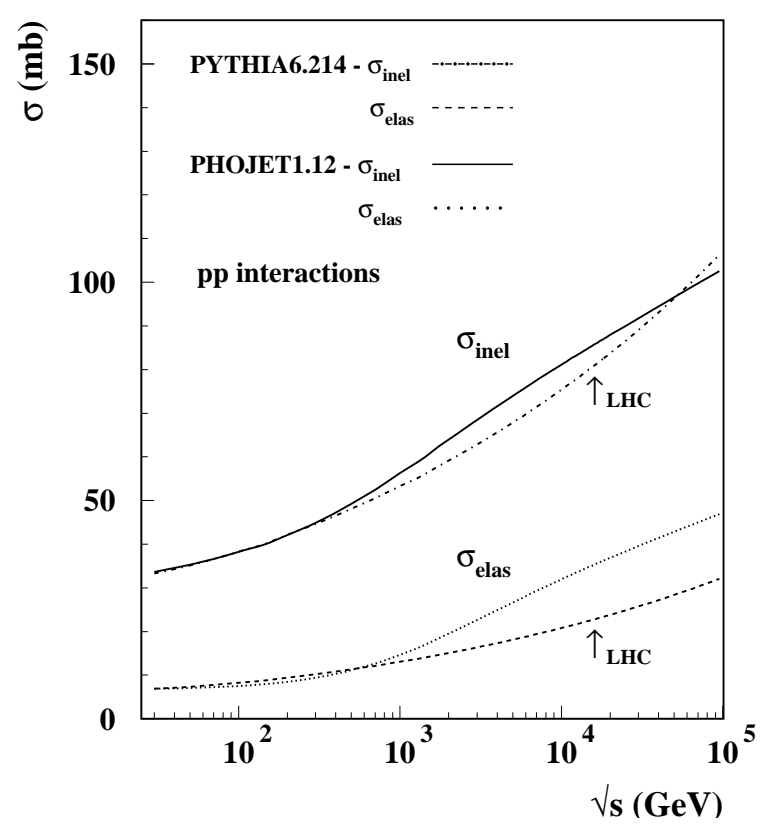

(a)

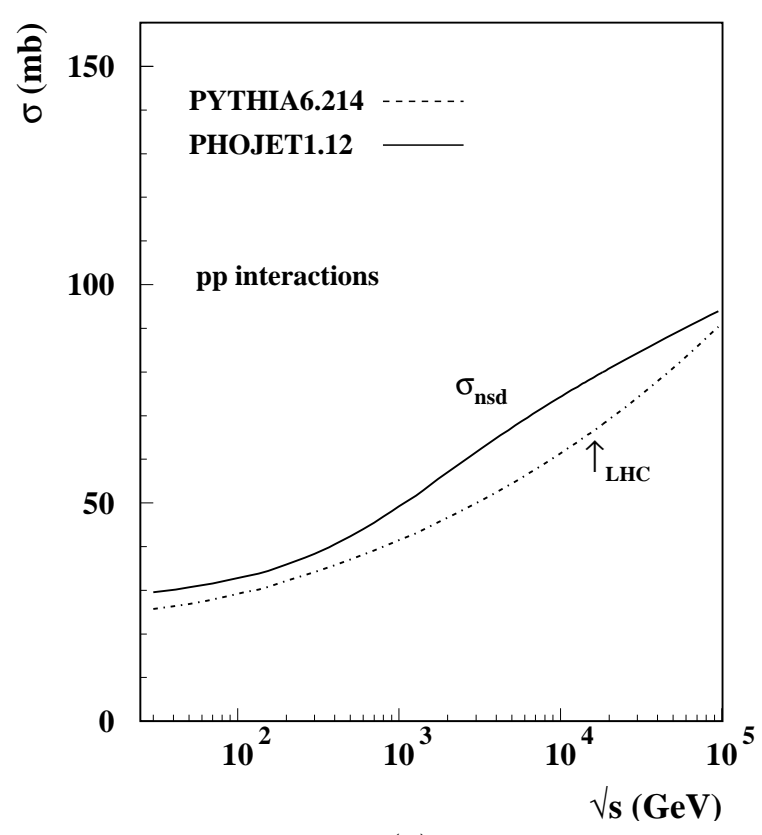

(b)

Fig. 5. PYTHIA6.214 and PHOJET1.12 cross-section predictions for pp collisions: (a) inelastic and elastic, and (b) NSD interactions.

processes generated as minimum bias events as this category of interactions also fits in the selection of NSD events.

In Appendix B we verify that the direct selection of NSD interactions in both PYTHIA6.214 and PHOJET1.12 (as indicated in table 2) agrees reasonably well to the cross-sections and minimum bias distributions predicted by the same event generators with the trigger simulation. 


\begin{tabular}{|c|c|c|}
\hline PYTHIA6.214 & $\begin{array}{c}\operatorname{MSUB}(94)=1 \\
\operatorname{MSUB}(95)=1\end{array}$ & $\begin{array}{c}\text { double diffraction } \\
\text { low- } \mathrm{p}_{t} \text { production }\end{array}$ \\
\hline PHOJET1.12 & $\begin{array}{c}\operatorname{IPRON}(1,1)=1 \\
\operatorname{IPRON}(4,1)=1 \\
\operatorname{IPRON}(7,1)=1\end{array}$ & $\begin{array}{c}\text { non-diffractive } \\
\text { central diffraction } \\
\text { double diffraction }\end{array}$ \\
\hline
\end{tabular}

Table 2. Parameters used to generate minimum bias events (NSD).

Defining minimum bias events as NSD interactions enables us to probe theoretical models by comparing them with a variety of experimental observables measured over a range of energies in the last quarter of a century.

Diffractive inelastic events (single and double diffractive) are simulated in both programs. Although different parametrizations are used, both PYTHIA and PHOJET consider Pomeron models to describe diffractive events $[12,22]$. The NSD cross-section, $\sigma_{n s d}$, is then obtained by subtracting single diffractive cross-sections from the inelastic.

Figure 5(b) shows the estimated $\sigma_{n s d}$ for pp collisions generated by PYTHIA 6.214 and PHOJET1.12. For the centre-of-mass energy range displayed in fig. 5(b), PHOJET generates higher cross-sections for non-single diffractive interactions, or minimum bias events, than PYTHIA. This implies that for pp collisions at the same luminosity, PHOJET will generate more minimum bias events than PYTHIA. The non-single diffractive scattering crosssections obtained for each generator at $\sqrt{s}=14 \mathrm{TeV}$ were: $65.7 \mathrm{mb}$ for PYTHIA6.214, and $73.8 \mathrm{mb}$ for PHOJET 1.12 , a difference of $12.3 \%$. This shows that minimum bias events are expected to contribute significantly (more than $65 \%$ ) to the total scattering process at the LHC.

\subsection{Charged particle multiplicity distribution}

Charged particle multiplicity distributions [17] have been widely used as important tools for studying multiple particle production in inelastic and NSD events $[13,14,16]$. They are particularly useful when displayed in terms of multiplicity scaled variables as suggested by Koba, Nielsen and Olesen (KNO variables) [17]. Plotted as a function of KNO variables, the charged multiplicity distributions provide a clearer display of fluctuations seen for both very low (less than half of the average multiplicity) or very high multiplicity (more than the double the average) events. This provides invaluable information on the evolution of the event multiplicity with the colliding energy.

When the KNO plots first appeared, it was suggested that the probability distributions, $\mathrm{P}_{n}$, of producing $\mathrm{n}$ particles at a given inelastic or minimum bias hadron-hadron collision should scale with energy as $s \rightarrow \infty$ following the

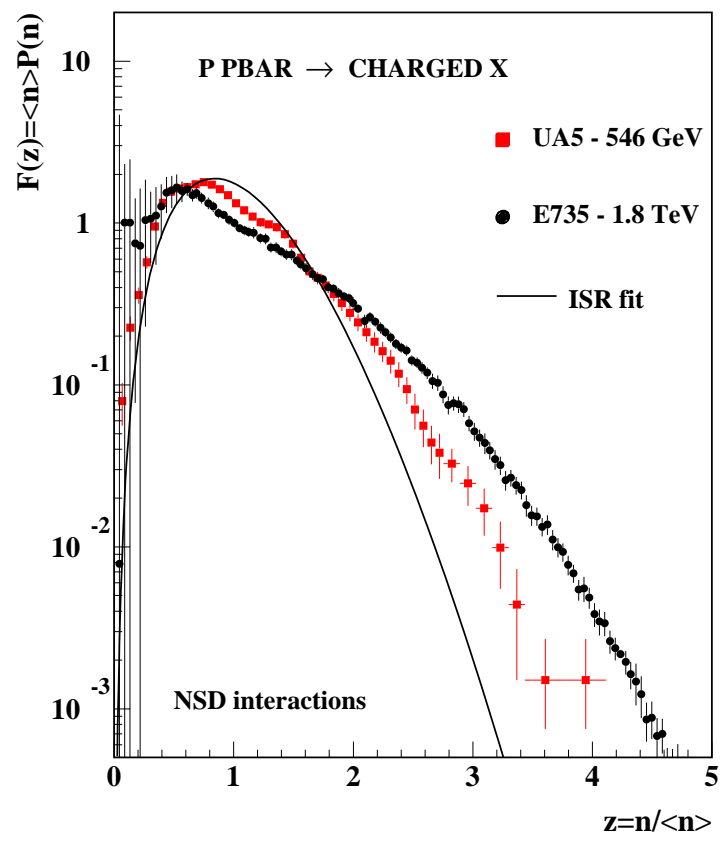

Fig. 6. Charged multiplicity distributions for $\mathrm{p} \overline{\mathrm{p}}$ collisions at $\sqrt{\mathrm{s}}=546 \mathrm{GeV}$ and $1.8 \mathrm{TeV}$, compared to the fit to ISR distributions.

relation

$$
F(z)=\langle n\rangle \mathrm{P}_{n}=\langle n\rangle \frac{\sigma_{n}}{\sum_{n} \sigma_{n}},
$$

with $\langle n\rangle$ being the average particle multiplicity, $\mathrm{z}=\mathrm{n} /\langle\mathrm{n}\rangle$ and $\mathrm{P}_{n}$ alternatively defined in terms of cross-sections as $\mathrm{P}_{n}=\sigma_{n} / \sum_{n} \sigma_{n}$, where $\sigma_{n}$ is the cross-section for interactions producing $\mathrm{n}$ particles and $\sum_{n} \sigma_{n}$ is the inelastic or the minimum bias cross-section.

KNO scaling was indeed observed up to the highest ISR energies as shown in Ref. [13]. Nevertheless, experiments with centre of mass energy greater than those achieved at the ISR demonstrated the scaling is broken $[14,18]$.

Figure 6 shows charged multiplicity distributions for NSD p $\overline{\mathrm{p}}$ collisions measured by UA5 at $\sqrt{\mathrm{s}}=546 \mathrm{GeV}[14]$ and by E735 at $\sqrt{\mathrm{s}}=1.8 \mathrm{TeV}[16]$. It also shows the fit to the KNO distributions measured at ISR given in Ref. [13].

Comparing the UA5 - $546 \mathrm{GeV}$ and E735 data to the ISR fit, the KNO violation is clearly visible. The violation occurs for $z>2$, i.e., for high multiplicity events. The scaling violation is interpreted as a manifestation of multiparton interactions (second, third or a higher number of parton scatterings) [7-9] whose effects become measurable as the colliding energy increases [16]. 


\subsection{Describing charged multiplicity distributions with PYTHIA}

By default PYTHIA is set to use multiple parton interactions. Nevertheless, one still has to define how the divergency for scatterings with $\mathrm{p}_{t}<\mathrm{p}_{t_{\text {min }}}$ will be treated by the event generator. PYTHIA allows two different phenomenological approaches: simple and complex scenarios [11]. Selecting the complex scenario one has also the choice of selecting different matter distributions for the colliding hadrons, which introduces a dependence on the collision's impact parameter $b$.

In this section we compare charged multiplicity distributions generated using the simple scenario $(\operatorname{MSTP}(82)$ $=1$ ) and those obtained using the complex scenario (MSTP $(82)=2,3$ and 4$)$ to the data. Comparisons between PYTHIA's models and the p $\overline{\mathrm{p}} 546 \mathrm{GeV}$ UA5 data have been presented in Ref. [11]. Here we extend these comparisons to UA5 data taken at $900 \mathrm{GeV}$ [14], to recent data taken at higher energies by E735 [16] and also to one of the latest PYTHIA versions [12].

For each comparison we calculate the $\chi^{2} /$ d.o.f. associated to the MC generated distribution as [31]

$$
\chi^{2} / \text { d.o.f. }=\frac{1}{N_{\text {d.o.f. }}} \sum_{i=1}^{N_{\text {d.o.f. }}} \frac{\left(y_{i}-\mu_{i}\right)^{2}}{\sigma_{i}^{2}\left(y_{i}\right)+\sigma_{i}^{2}\left(\mu_{i}\right)},
$$

where $\mathrm{N}_{\text {d.o.f. }}$ is the number of degrees of freedom, $\mathrm{y}_{i}$ and $\mu_{i}$ are the MC and measured values for the ith bin respectively, and $\sigma^{2}\left(y_{i}\right)$ and $\sigma^{2}\left(\mu_{i}\right)$ are the errors associated to $\mathrm{y}_{i}$ and $\mu_{i}$. This allows us to compare how well simulations generated with different parameters agree with the data.

Figure 7 shows charged multiplicity distributions for NSD p $\overline{\mathrm{p}}$ collisions at $\sqrt{\mathrm{s}}=546 \mathrm{GeV}$ and $1.8 \mathrm{TeV}$. We compare distributions generated by PYTHIA's simple and complex scenarios to UA5 [14] and E735 [16] data. Apart from the mentioned changes in the setting $\operatorname{MSTP}(82)$, all other parameters are set to use PYTHIA's default options, as described in [12]. Typically, the number of generated events used to plot each MC distribution is greater than $10^{6}$ events. This is valid not only for charged particle distributions but also for all minimum bias predictions shown in this note.

Figures 7(a) and (b) show that using the simple scenario $(\operatorname{MSTP}(82)=1)$, which is the default in PYTHIA 6.214 [12], the generated distributions fail to reproduce the data, especially in the region of high $\mathrm{z}(\mathrm{z}>1.5)$. This is the region of events with particle multiplicities several times greater than the average multiplicity. The $\chi^{2} /$ d.o.f. associated to the MC generated distributions in figs. 7(a) and (b) are $\chi^{2} / 47$ d.o.f. $=80.6$ and $\chi^{2} / 128$ d.o.f. $=107.2$, respectively. Notice that although some points in the MC distributions may be off the curve and look like a systematic effect in the model predictions they are actually the result of using the binning of the data (which depends on the measurement of $\mathrm{z}=\mathrm{n} /\langle\mathrm{n}\rangle)$ to produce the MC plots. This, however, has no effect on the final results.

Distributions generated using the complex scenario vary with the hadronic matter distribution selected for each case. As shown in figs. 7(c) and (d), the complex scenario option which uses a uniform hadronic matter distribution $(\operatorname{MSTP}(82)=2)$ fails to describe the charged multiplicity distributions correctly and gives $\chi^{2} / 47$ d.o.f. $=$ 86.2 and $\chi^{2} / 128$ d.o.f. $=132.9$, for the distributions at $546 \mathrm{GeV}$ and $1.8 \mathrm{TeV}$, respectively. Notice that for both the simple scenario and the complex scenario with a uniform matter distribution, the charged multiplicity distributions tend to have a Poissonian behaviour and cannot reproduce the shape of the high-multiplicity tail. The distributions generated using the double Gaussian option $(\operatorname{MSTP}(82)=4)$ also fail to reproduce the data and generate an excess of high multiplicity events, which results in $\chi^{2} / 47$ d.o.f. $=84.3$ and $\chi^{2} / 128$ d.o.f. $=66.9$, respectively. The best agreement to the data is observed for the complex scenario with the single Gaussian matter distribution option $(\operatorname{MSTP}(82)=3)$. The $\chi^{2} /$ d.o.f. obtained with this option are $\chi^{2} / 47$ d.o.f. $=33.9$ and $\chi^{2} / 128$ d.o.f. $=29.1$ for the comparisons shown in figs. 7 (c) and (d).

The comparisons of charged multiplicity distributions shown in figs. 7(c) and (d) indicate that the matter distribution used to describe the colliding hadrons does affect the probability of particle production in minimum bias events. The simple Poisson distribution of multi-parton events fail to fit the data indicating that there is a correlation between impact parameter and number of events. This was also seen as the pedestal effect by UA1 [11].

Although in the comparisons shown in fig.7, where all PYTHIA parameters except MSTP(82) are kept as default, the best agreement to the data was obtained by selecting the complex scenario with the single Gaussian matter distribution option, we shall adopt the complex scenario with a double Gaussian matter distribution (MSTP $(82)=4)$ as our preferred choice.

This is done because by choosing the double Gaussian option, the user is able to control some of the properties of this matter distribution. The double Gaussian matter distribution is given by [12]

$$
\rho(r) \propto \frac{1-\beta}{a_{1}^{3}} \exp \left\{-\frac{r^{2}}{a_{1}^{2}}\right\}+\frac{\beta}{a_{2}^{3}} \exp \left\{-\frac{r^{2}}{a_{2}^{2}}\right\}
$$

Hadrons described by this distribution have a small core region of radius $a_{2}$ containing a fraction $\beta$ of the total hadronic matter. This core is embedded in a larger volume of radius a $a_{1}$ containing the remaining fraction of matter, i.e., $(1-\beta)$ of the total hadronic matter. The parameter PARP (83) controls the portion $\beta$ of the total hadronic matter assigned to the core of the hadron. The ratio $a_{2} / a_{1}$ is given by the parameter PARP $(84)$. By default, PYTHIA sets $\operatorname{PARP}(83)=0.5$ and $\operatorname{PARP}(84)=0.2$ describing any given hadron as a body with half of its matter concentrated within a core which is limited by a radius $\mathrm{a}_{2}=$ $20 \%$ of the hadron radius a $a_{1}[12]$.

Notice that this double Gaussian distribution can easily be reduced to a single Gaussian by selecting specific values of $\operatorname{PARP}(83)$ or $\operatorname{PARP}(84)$ (e.g. $\operatorname{PARP}(83)=1$ or $\operatorname{PARP}(84)=1)$.

High $\mathrm{p}_{t}$ scattering processes are associated with small impact parameters. Therefore if a double gaussian model 


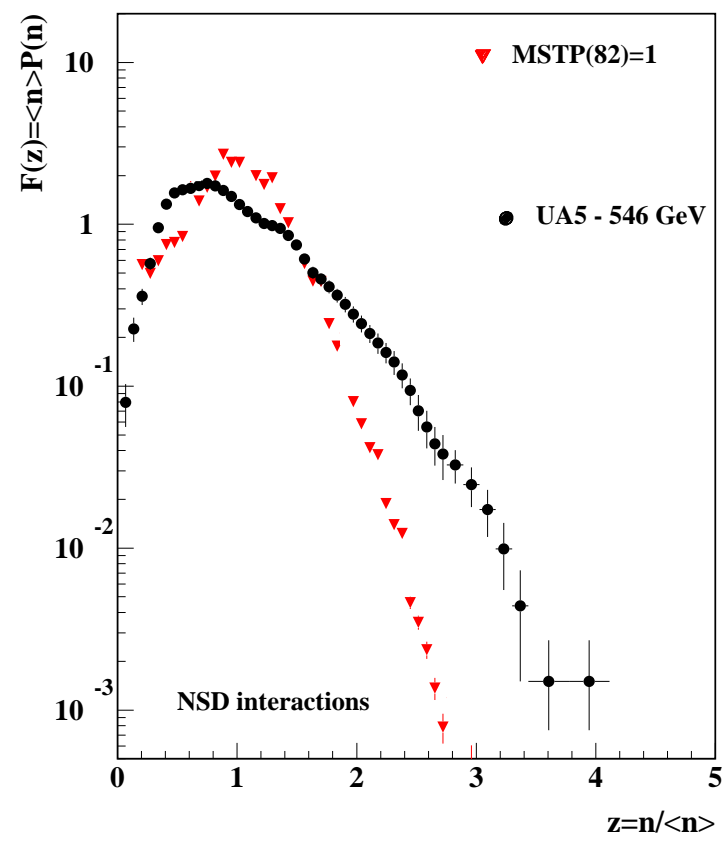

(a)

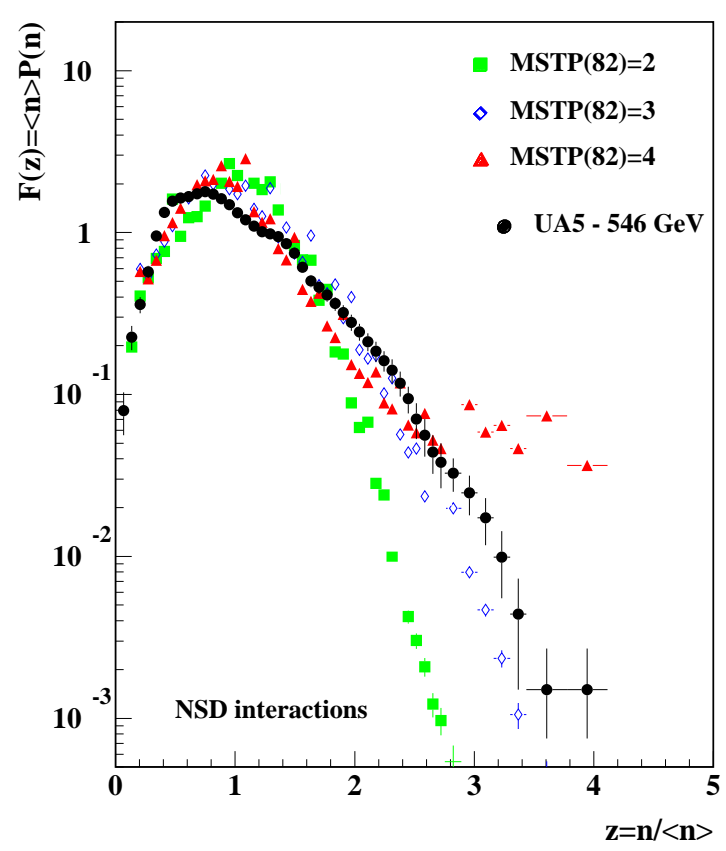

(c)

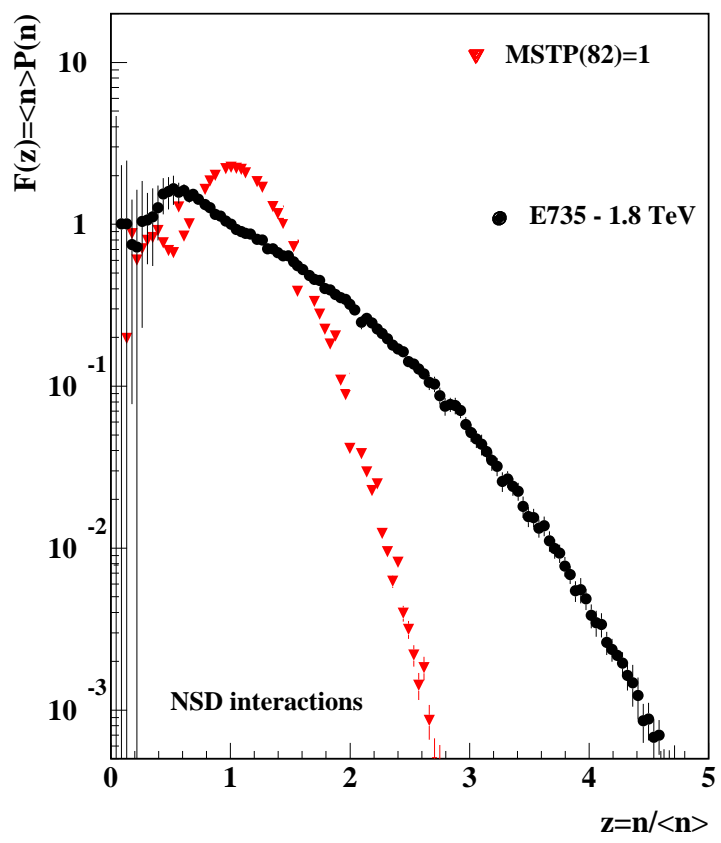

(b)

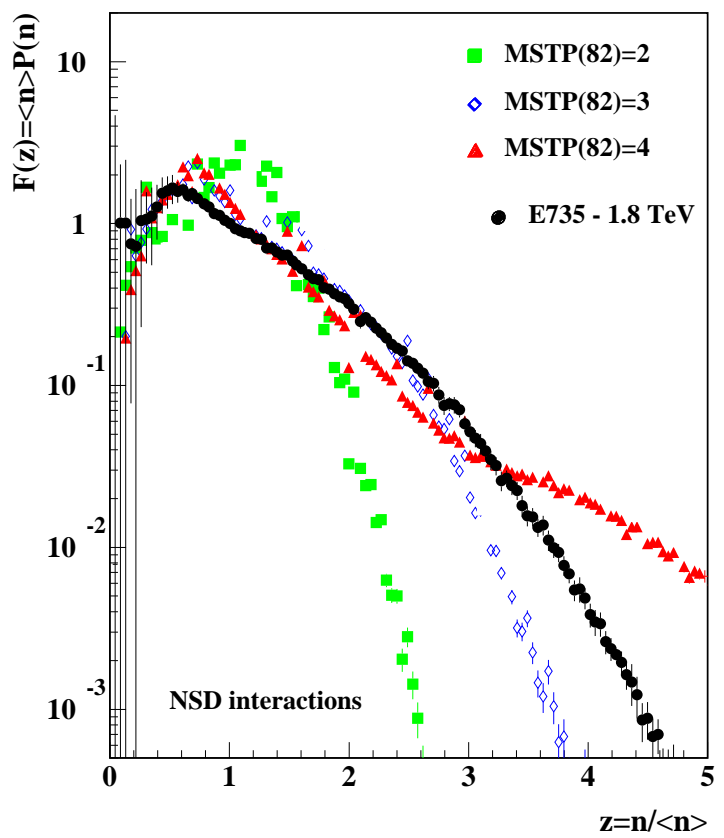

(d)

Fig. 7. Charged multiplicity distributions for NSD p $\overline{\mathrm{p}}$ collisions at $\sqrt{\mathrm{s}}=546 \mathrm{GeV}$ and $1.8 \mathrm{TeV}$ : (a) and (b) simple scenario distributions compared to data for $\sqrt{\mathrm{s}}=546 \mathrm{GeV}$ and $1.8 \mathrm{TeV}$, respectively; (c) and (d) complex scenario distributions compared to data for $\sqrt{\mathrm{s}}=546 \mathrm{GeV}$ and $1.8 \mathrm{TeV}$, respectively. 


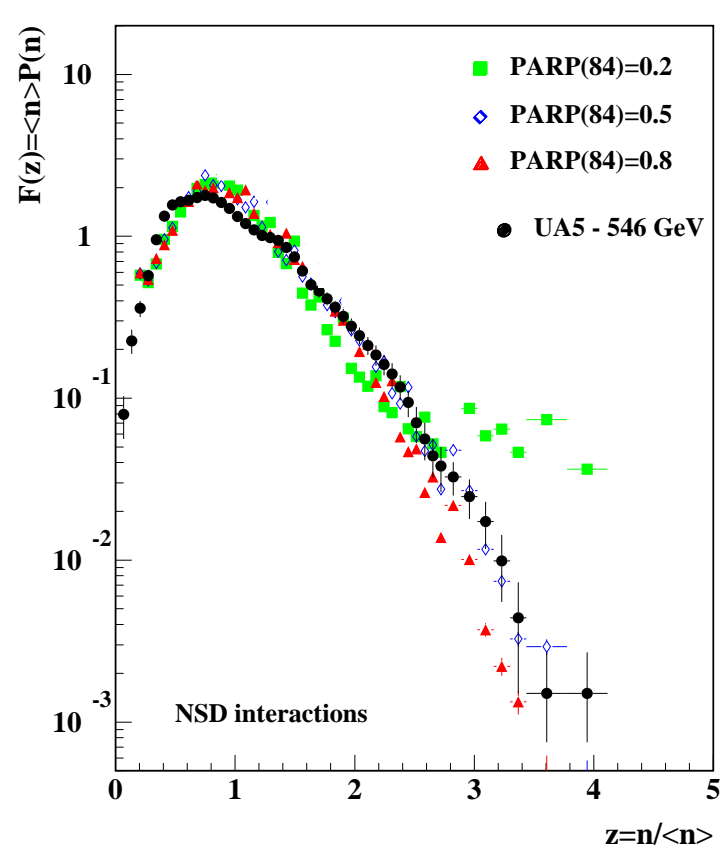

(a)

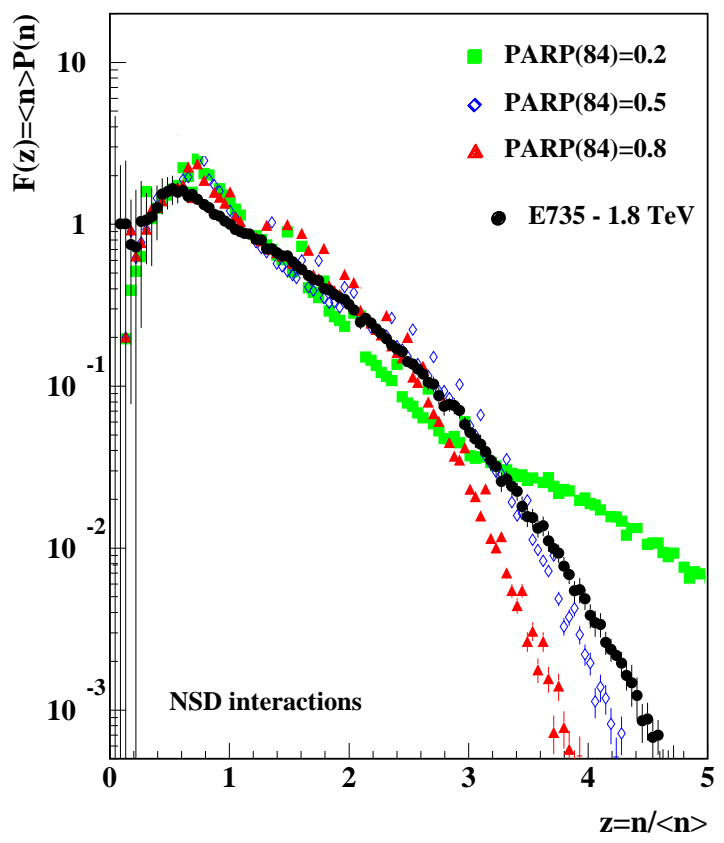

(b)

Fig. 8. Charged multiplicity distributions for NSD p $\bar{p}$ collisions at (a) $\sqrt{\mathrm{s}}=546 \mathrm{GeV}$ and (b) $\sqrt{\mathrm{s}}=1.8 \mathrm{TeV}$.

with a dense core is used to describe the matter distribution in the proton, hard scatters will be associated with a greater number of multiple interactions and hence a greater activity in the underlying event.

\subsubsection{Double Gaussian matter distribution}

As noted above, the type of matter distribution describing the colliding hadrons produces considerable changes in the shape of the charged multiplicity distributions (figs. 7(c) and (d) ). The effect produced by variations in the properties of the double Gaussian distribution, particularly the effect of variations in the core size to the minimum bias charged multiplicity distributions can be seen in fig.8.

As shown in fig. 8 considerable changes in the high-z tail of the charged multiplicity distributions are observed as the core radius varies from $20 \%$ to $50 \%$ and $80 \%$ of the radius of the colliding hadrons. As the core is made harder and denser (smaller core radius) the overlap between two colliding cores is accompanied by high- $\mathrm{p}_{t}$ partonic scatterings, and yields higher multiplicity events more often than when two relatively softer cores (larger radius) overlap in a collision.

For the distributions generated at $\sqrt{\mathrm{s}}=546 \mathrm{GeV}$, the $\chi^{2} /$ d.o.f. varies from $\chi^{2} / 47$ d.o.f. $=84.3$ to 30.8 and 34.9 as PARP (84) varies from 0.2 to 0.5 and 0.8 , respectively. At $\sqrt{\mathrm{s}}=1.8 \mathrm{TeV}, \chi^{2} / 128$ d.o.f. $=66.9,17.8$ and 34.4 for $\operatorname{PARP}(84)=0.2,0.5$ and 0.8 , respectively. This indicates that the choice of a value for the core size affects considerably how well charged multiplicity distributions are described by PYTHIA. Notice that in PYTHIA6.214 the default value for $\operatorname{PARP}(84)$ is 0.2 , which is the parameter giving the worse $\chi^{2} / d$.o.f. for the comparisons shown in fig. 8. The data favour a larger less dense core than the default option currently used by PYTHIA6.214.

\subsection{Describing charged multiplicity distributions with PHOJET}

As mentioned above, PHOJET describes hadron collisions using the multiple Pomeron exchange mechanism proposed by the DPM [10] complemented by a multiple parton scattering picture for high- $\mathrm{p}_{t}$ processes [22]. Figure 9 shows charged multiplicity distributions for NSD p $\bar{p}$ collisions at $\sqrt{\mathrm{s}}=546 \mathrm{GeV}$ and $1.8 \mathrm{TeV}$, comparing distributions generated by PHOJET1.12 to UA5 [14] and E735 [16] data.

There is a reasonable agreement between charged multiplicity distributions generated by PHOJET1.12 and the data. The $\chi^{2} /$ d.o.f. obtained by comparing PHOJET1.12 to the data shown in figs. 9 (a) and (b) are $\chi^{2} / 47$ d.o.f. $=$ 11.8 and $\chi^{2} / 128$ d.o.f. $=10.6$, respectively for $\sqrt{\mathrm{s}}=546$ $\mathrm{GeV}$ and $1.8 \mathrm{TeV}$.

\subsection{Pseudorapidity distribution}

The rate of parton-parton scattering in a hadronic collision is strongly correlated to the observed particle multiplicity and the pseudorapidity distribution of produced particles. This happens because in each parton interaction part of the collision energy that would otherwise be carried by the fast moving system of beam-remnants in the 


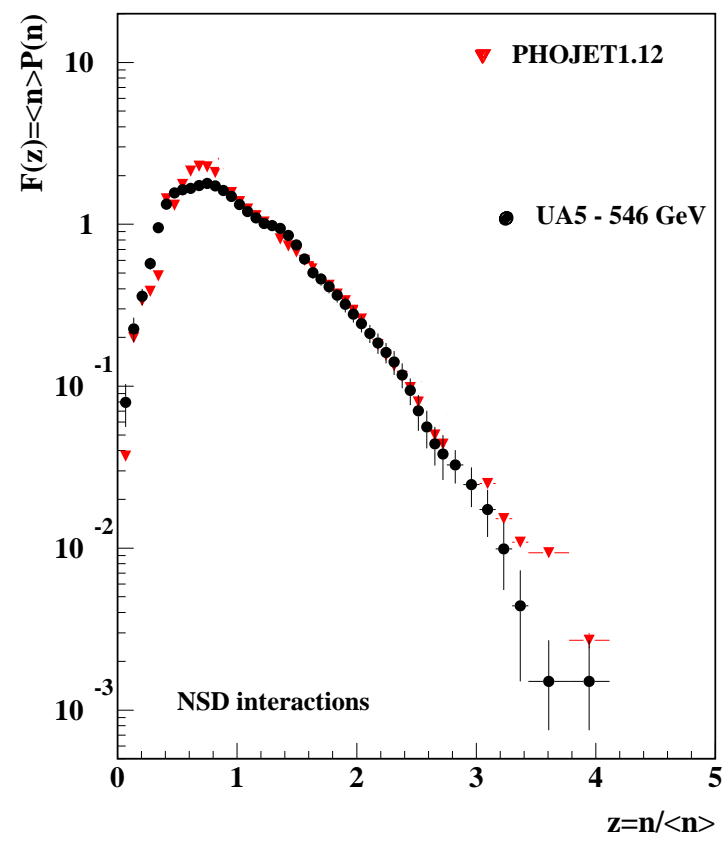

(a)

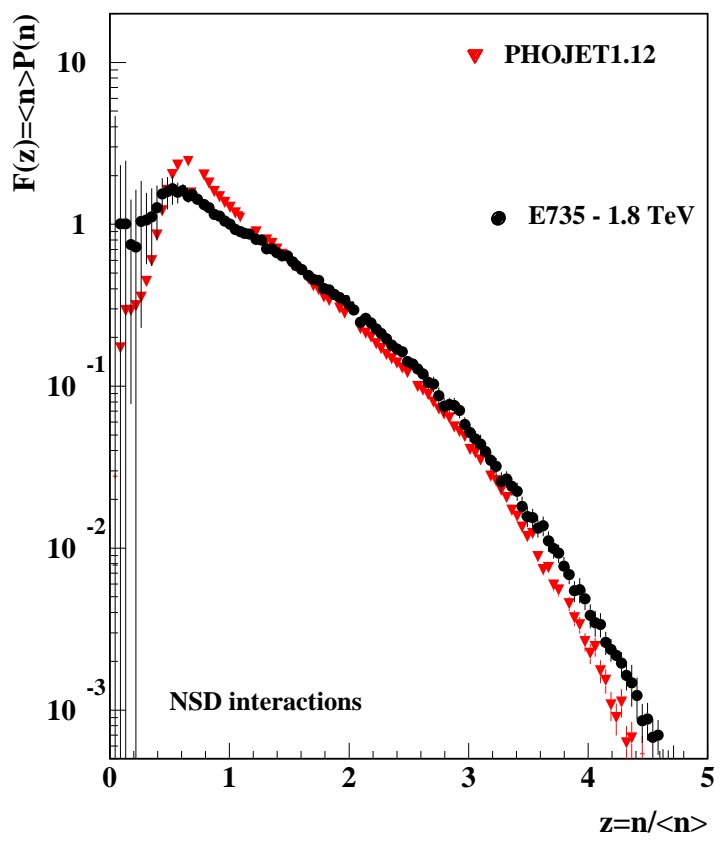

(b)

Fig. 9. Charged multiplicity distributions for NSD p $\bar{p}$ collisions at (a) $\sqrt{\mathrm{s}}=546 \mathrm{GeV}$ and (b) $\sqrt{\mathrm{s}}=1.8 \mathrm{TeV}$.

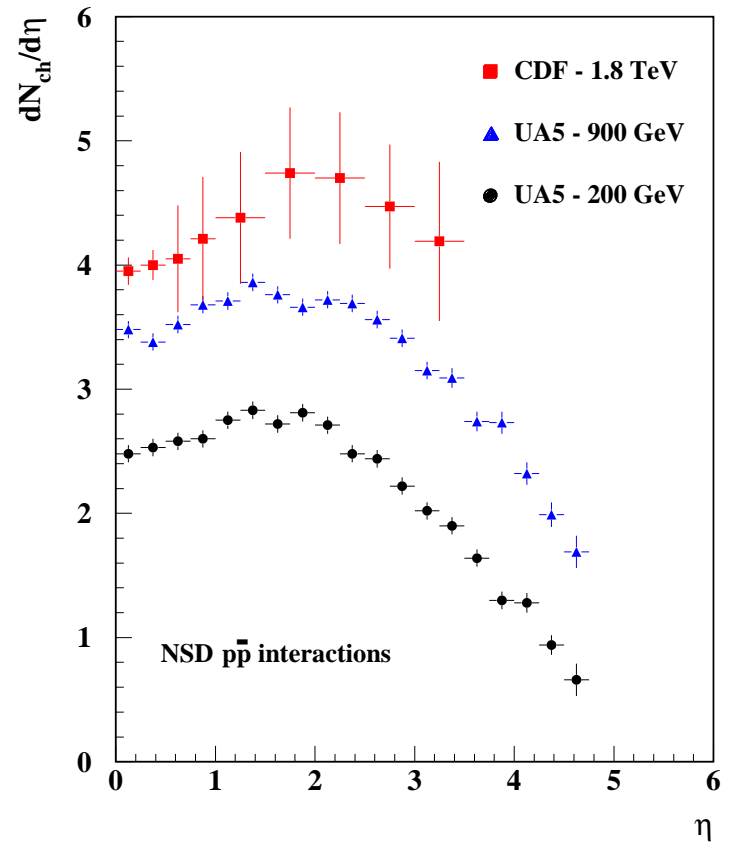

Fig. 10. Charged particle density distributions for NSD $p \bar{p}$ collisions at $\sqrt{\mathrm{s}}=200 \mathrm{GeV}$ [19], $900 \mathrm{GeV}$ [19] and 1.8 TeV [15].

forward regions, is converted into low- $\mathrm{p}_{t}$ particles which populate the central region.

Figure 10 displays charged particle densities, $\mathrm{dN}_{c h} / \mathrm{d} \eta$, distributed in the pseudorapidity space, $\eta$, for NSD $p \bar{p}$ collisions at $\sqrt{\mathrm{s}}=200 \mathrm{GeV}$ [19], $900 \mathrm{GeV}$ [19] and 1.8 $\mathrm{TeV}$ [15]. It shows a central plateau at small $\eta$ and a falling density in the fragmentation region, i.e. $\eta \rightarrow \eta_{\max }$. As the colliding energy increases, the rate of multiple parton interactions, or multiple Pomeron exchanges, also increases producing a rise on the central plateau. Therefore, in order to correctly describe $\mathrm{dN}_{c h} / \mathrm{d} \eta$, the MC event generators have to generate the right amount of partonic activity (multiple parton scattering or multiple Pomeron exchanges), taking into account the expected variation with the colliding energy.

In PYTHIA, one of the main parameters used to regulate the rate of parton-parton interactions is $\mathrm{p}_{t_{\min }}$ given by eq.(8). Low values of $\mathrm{p}_{t_{\text {min }}}$ imply high rates of partonparton scatterings and hence in high levels of particle multiplicity; for increasing $\mathrm{p}_{t_{\text {min }}}$ the opposite is expected.

Figure 11 displays charged particle density distributions, $\mathrm{dN}_{c h} / \mathrm{d} \eta$, plotted against pseudorapidity, $\eta$. It shows a comparison between UA5 and CDF measurements of $\mathrm{dN}_{c h} / \mathrm{d} \eta[15,19]$ (figs. 11 (a) and (b), respectively) and distributions generated by PYTHIA6.214 with the complex scenario for multiple parton scattering $(\operatorname{MSTP}(82)=4)$ varying $\mathrm{p}_{t_{\text {min }}}$ by changing the parameter $\operatorname{PARP}(82)$. As expected, increasing PARP(82) from 1.7 to 1.9 and 2.1, i.e. increasing $\mathrm{p}_{t_{m i n}}, \mathrm{dN}_{c h} / \mathrm{d} \eta$ decreases.

For the $\mathrm{dN}_{c h} / \mathrm{d} \eta$ distributions generated by PYTHIA 6.214 at $\sqrt{\mathrm{s}}=900 \mathrm{GeV}, \chi^{2} / 19$ d.o.f. $=25.2,1.9$ and 13.4 for $\operatorname{PARP}(82)=1.7,1.9$ and 2.1 , respectively. At $\sqrt{\mathrm{s}}$ $=1.8 \mathrm{TeV}, \chi^{2} / 9$ d.o.f. $=5.6,0.3$ and 1.8 for $\operatorname{PARP}(82)$ $=1.7,1.9$ and 2.1 , respectively. Notice that relatively small changes in $\operatorname{PARP}(82)(\sim 10 \%)$ cause significant variations 


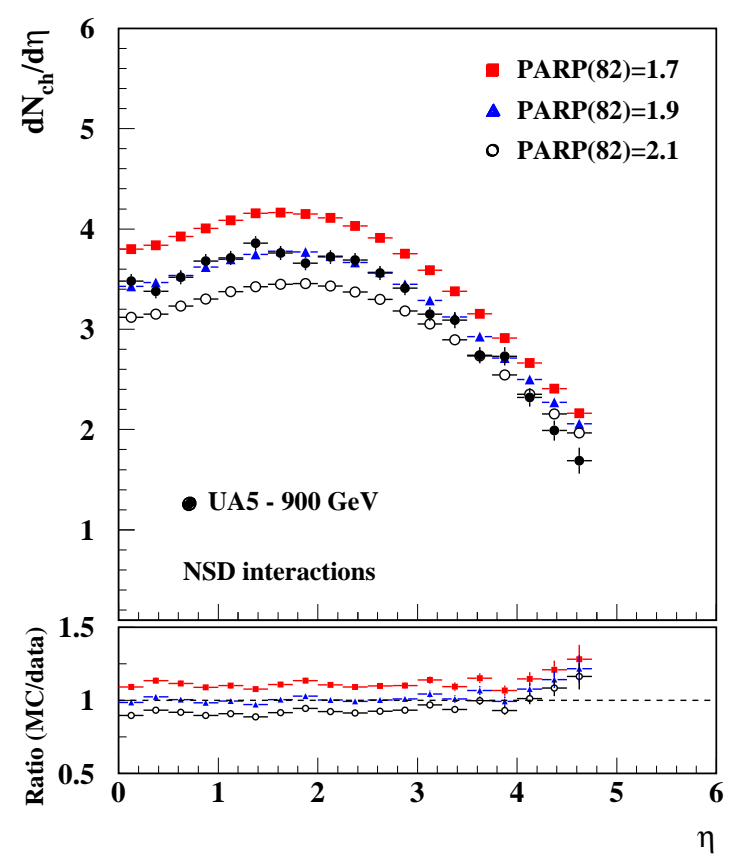

(a)

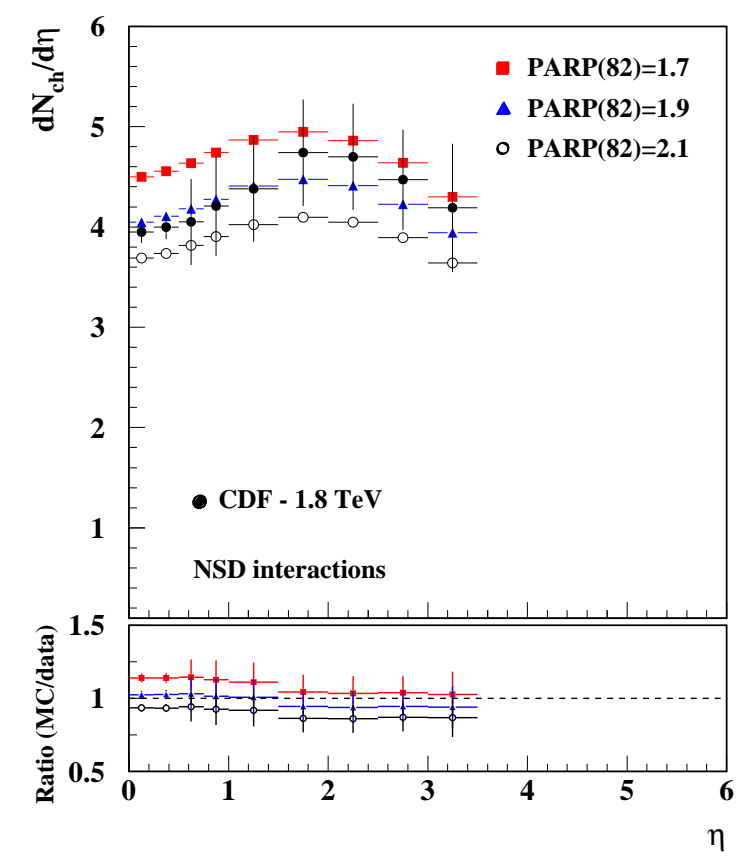

(b)

Fig. 11. Charged particle density distributions, $\mathrm{dN}_{c h} / \mathrm{d} \eta$, for NSD p $\overline{\mathrm{p}}$ collisions comparing PYTHIA6.214 to the data at (a) $\sqrt{\mathrm{s}}$ $=900 \mathrm{GeV}$ and $(\mathrm{b}) \sqrt{\mathrm{s}}=1.8 \mathrm{TeV}$.

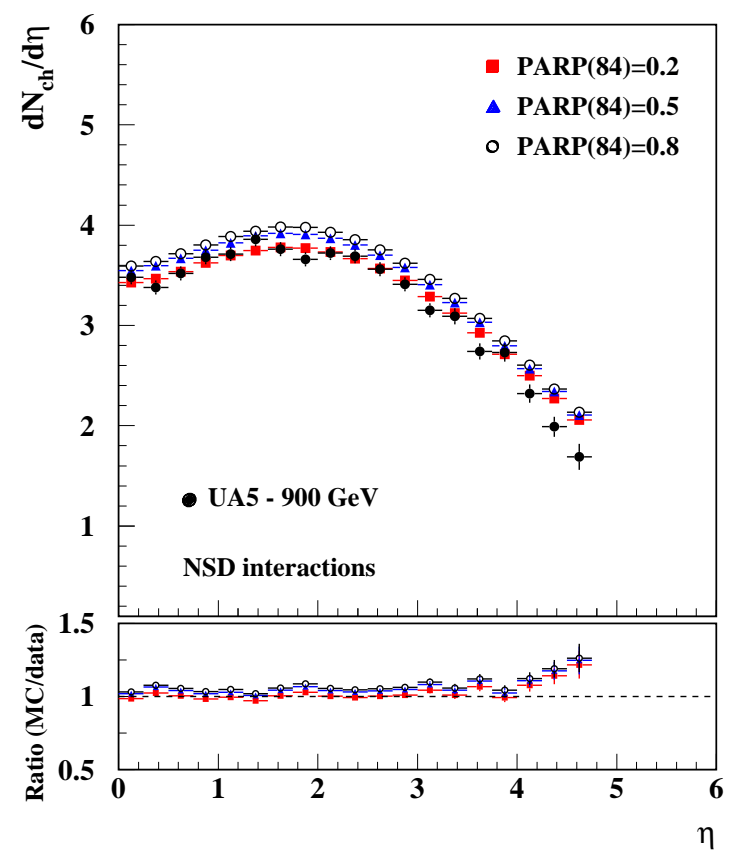

(a)

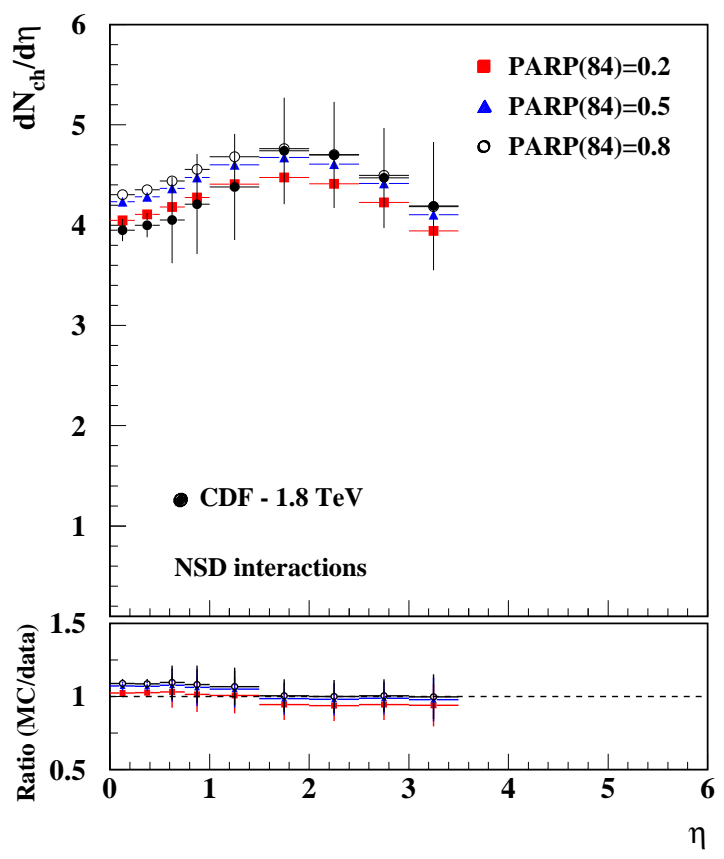

(b)

Fig. 12. Charged particle density distributions, $\mathrm{dN}_{c h} / \mathrm{d} \eta$, for NSD p $\overline{\mathrm{p}}$ collisions comparing PYTHIA6.214 for different core-sizes to the data at (a) $\sqrt{\mathrm{s}}=900 \mathrm{GeV}$ and (b) $\sqrt{\mathrm{s}}=1.8 \mathrm{TeV}$. 


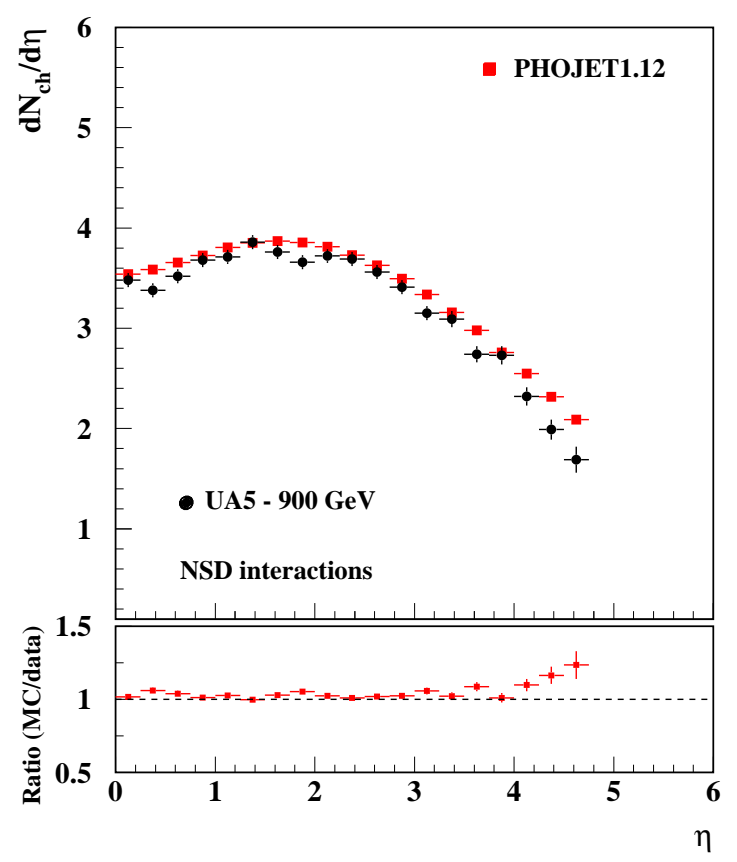

(a)

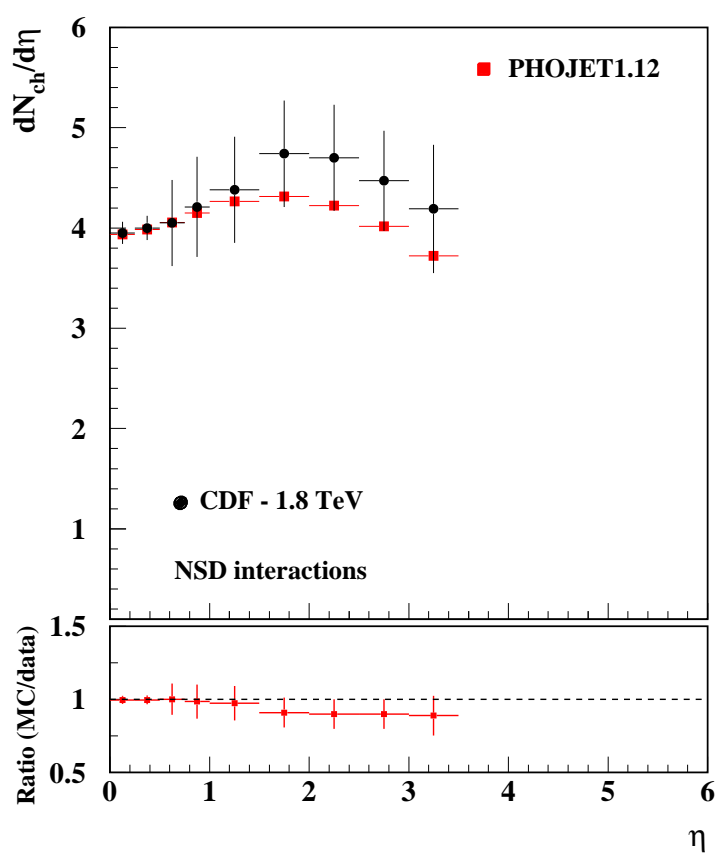

(b)

Fig. 13. Charged particle density distributions, $\mathrm{dN}_{c h} / \mathrm{d} \eta$, for NSD p $\overline{\mathrm{p}}$ collisions comparing PHOJET1.12 to the data at (a) $\sqrt{\mathrm{s}}$ $=900 \mathrm{GeV}$ and $(\mathrm{b}) \sqrt{\mathrm{s}}=1.8 \mathrm{TeV}$.

in $\chi^{2} /$ d.o.f. showing how sensitive $\mathrm{dN}_{c h} / \mathrm{d} \eta$ distributions generated by PYTHIA are to changes in $\mathrm{p}_{t_{\min }}$. The default value of $\operatorname{PARP}(82)$ is set to 1.9 in PYTHIA6.214.

The charged particle density $\mathrm{dN}_{c h} / \mathrm{d} \eta$ is also sensitive to the parameters chosen for the core-size $\operatorname{PARP}(84)$. In fig. $12 \mathrm{dN}_{c h} / \mathrm{d} \eta$ distributions generated by PYTHIA6.214 with $\operatorname{PARP}(84)=0.2,0.5$ and 0.8 are compared to UA5 (NSD p $\overline{\mathrm{p}}$ collisions at $\sqrt{\mathrm{s}}=900 \mathrm{GeV} 12(\mathrm{a})$ ) and CDF data (NSD p $\overline{\mathrm{p}}$ collisions at $\sqrt{\mathrm{s}}=1.8 \mathrm{TeV} 12(\mathrm{~b})$ ). In 12 (a) the obtained $\chi^{2} / 19$ d.o.f. is $1.9,5.9$ and 9.2 , for $\operatorname{PARP}(84)=0.2,0.5$ and 0.8 , respectively. For the comparisons shown in $12(\mathrm{~b})$, the $\chi^{2} / 9$ d.o.f. is $0.3,1.5$ and 2.3 for $\operatorname{PARP}(84)=0.2,0.5$ and 0.8 , respectively. Notice that although the core radius has been significantly varied ( $\sim$ factor of 2 for each subsequent increase), the relative changes in the $\chi^{2} /$ d.o.f. are much less dramatic than the ones seen for the comparatively smaller changes in $\mathrm{p}_{t_{m i n}}$ ( 10\% for each subsequent increase).

In PHOJET, multiple Pomeron exchanges are predicted by the DPM. Similarly to PYTHIA, this model also depends on a $p_{t}^{\text {cut-off }}$ which is used to connect the soft and hard components of a hadronic interaction. PHOJET1.12 has its default options tuned for $\mathrm{p}_{t}^{\text {cut-off }}=2.5 \mathrm{GeV}$. Figure 13 shows $\mathrm{dN}_{c h} / \mathrm{d} \eta$ distributions generated by PHOJET1.12 with its default cuts, compared to UA5 and CDF data in figs. 13 (a) and (b), respectively. There is a good agreement between PHOJET1.12 predictions and the data. The $\chi^{2} /$ d.o.f. are $\chi^{2} / 19$ d.o.f. $=3.8$ and $\chi^{2} / 9$ d.o.f. $=$ 0.3 , respectively, for the comparisons shown in figs. 13 (a) and (b).

\subsection{PHOJET and the Two-Component Model}

The comparisons of multiplicity and particle density distributions shown in the previous sections strongly indicate that the number of multiple parton or multiple Pomeron interactions can fundamentally change the agreement between model predictions and the data. While PYTHIA allows the user to tune the rate of multiple parton interactions by changing parameters which are rather independent of each other (e.g. $\mathrm{p}_{t_{\text {min }}}$ and the hadronic matter distribution) the same does not apply to PHOJET.

As discussed in section 2.2, PHOJET has been developed as a two-component model, i.e. soft and hard interactions are generated using different phenomenological approaches and then combined with the use of unitarity considerations: soft hadronic interactions are generated with a model based on DPM whereas hard processes are generated by models based on perturbative QCD [22]. The total event activity (hadronic cross-sections, rate of Pomeron and parton interactions, multicity and $\mathrm{p}_{t}$ distributions) is then obtained by connecting the soft and hard regions at an appropriate $\mathrm{p}_{t}^{\text {cut-off }}$.

The choice of $\mathrm{p}_{t}^{\text {cut-off }}=2.5 \mathrm{GeV}$ as default in PHOJET1.12 also implies that other model parameters, especially those related to the parametrization of Regge phenomenology for soft processes (Pomeron and Reggeon couplings for example), are tuned based on the choice of $\mathrm{p}_{t}^{\text {cut-off }}[10,22,25]$. Therefore, in order to use different values of $\mathrm{p}_{t}^{\text {cut-off }}$ one is required to re-tune the entire model.

A clear example of how varying only $\mathrm{p}_{t}^{\text {cut-off }}$ in PHOJET1.12 affects the physics it describes can be seen in 


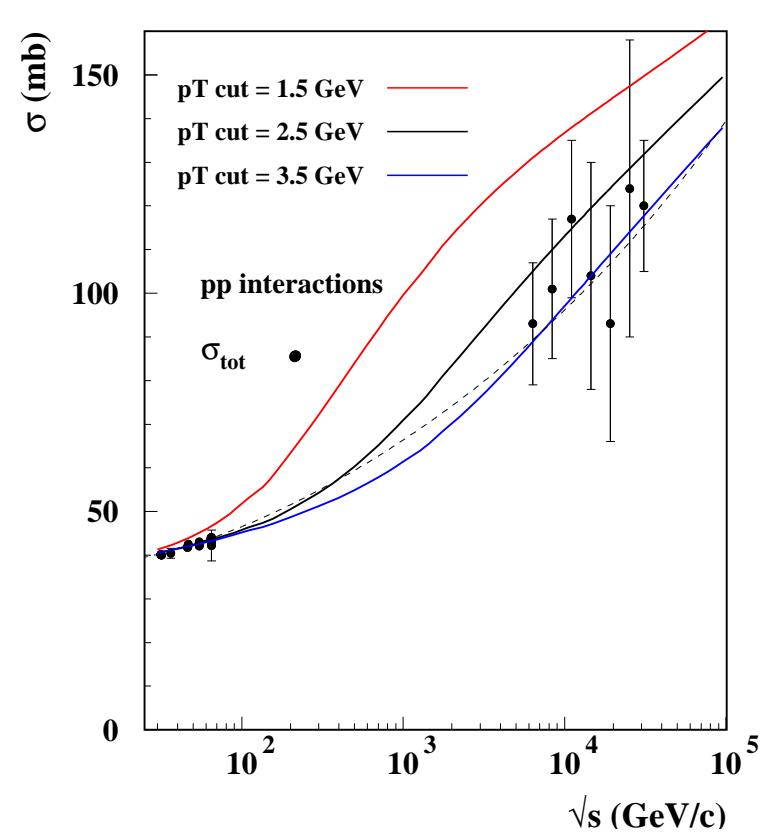

Fig. 14. Total cross-section for pp interactions predicted by PHOJET1.12 with different values of $\mathrm{p}_{t}^{\text {cut-off }}$. Data points taken from Ref. [30].

figure 14. Figure 14 displays the total cross-section for pp interactions predicted by PHOJET1.12 with different values of $\mathrm{p}_{t}^{\text {cut-off }}$. Note that varying $\mathrm{p}_{t}^{\text {cut-off }}$ results in considerable changes in the total cross-section. This occurs because by changing $\mathrm{p}_{t}^{\text {cut-off }}$ the balance between soft and hard interactions also changes and the program needs retuning.

Thus, the correct procedure to tune PHOJET1.12 would include not only changes to $\mathrm{p}_{t}^{\text {cut-off }}$ but also to the parametrization associated with the DPM for soft interactions.

\section{The underlying event}

In a hadronic event containing jets, the underlying event (UE) consists of all event activity except the high- $\mathrm{p}_{t}$ jets from the hard scattering process [20]. The underlying structure of hadronic interactions has not been fully understood yet and it is not clear how it should be modelled. As for minimum bias events, soft interactions play an important role in the structure of the underlying event and ought to be carefully considered by any model attempting to describe the underlying event.

Analyses developed by the CDF Collaboration indicate that the underlying event contains soft and hard components. The soft component is mainly associated with beam-beam remnant interactions. Particles composing the hard component come from the initial and final state radiation, from colour strings stretching between the underlying event and the highest- $\mathrm{p}_{t}$ jet and from secondary parton interactions. As for minimum bias studies, multiple parton interaction plays an important role in describing the event activity in the underlying event [20].

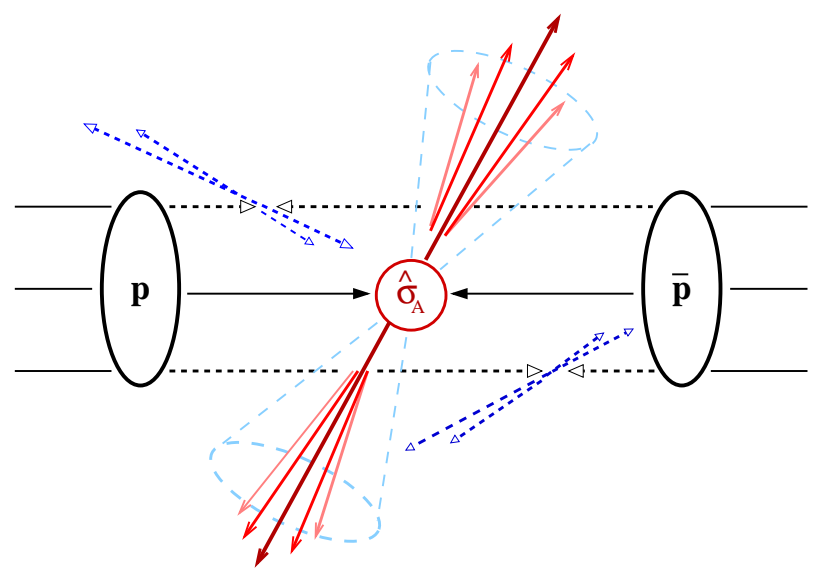

Fig. 15. Illustration of a jet produced by a hard parton-parton scattering in a $\mathrm{p} \overline{\mathrm{p}}$ collision.

To compare to the underlying event data presented in [20], a set of cuts must be applied to the MC simulation: (a) only charged particles are considered in this analysis; (b) selected particles must have $p_{t}>0.5 \mathrm{GeV}$ and $|\eta|<$ 1 [20]. A 92\% track finding efficiency is also applied to account for the tracking efficiency in CDF.

Once the particles were selected, the next step is to start looking for jets. The jet finder algorithm chosen was the cone jet finder in which jets are defined as circular regions in $\eta-\phi$ space with radius defined by

$$
R=\sqrt{(\Delta \eta)^{2}+(\Delta \phi)^{2}}
$$

where $\eta$ is the pseudorapidity and $\phi$ is the azimuthal scattering angle. The cone radius used in this analysis is $\mathrm{R}=0.7$.

The transverse momentum of a charged particle jet is defined as the scalar sum of the transverse momenta of the charged particles making up the jet. The jet with highest transverse momentum is taken to be the "leading charged particle jet", referred to as the leading jet.

The leading jet is used to define the event. Three regions are defined in terms of the azimuthal angle between charged particles and the leading charged jet. This angular difference is given by $\Delta \phi=\phi_{\text {particle }}-\phi_{\text {ljet }}$. The region $|\Delta \phi|<60^{\circ}$ is referred to as toward the leading charged jet and the region $|\Delta \phi|>120^{\circ}$ is called away from the leading jet. The region transverse to the leading jet is defined by $60^{\circ}<|\Delta \phi|<120^{\circ}$, and is used to study the underlying event. The event regions defined by $\Delta \phi$ are illustrated in fig. 16 .

In the following sections we compare PYTHIA6.214 with its default parameters as well as with the double Gaussian option for the complex scenario $(\operatorname{MSTP}(82)=4)$, and PHOJET1.12 to the CDF data for charged jet evolution, focusing on the distributions associated to the underlying event.

\subsection{Describing the UE with PYTHIA}

In PYTHIA the activity in the underlying event is related to the number of multiparton interactions. 


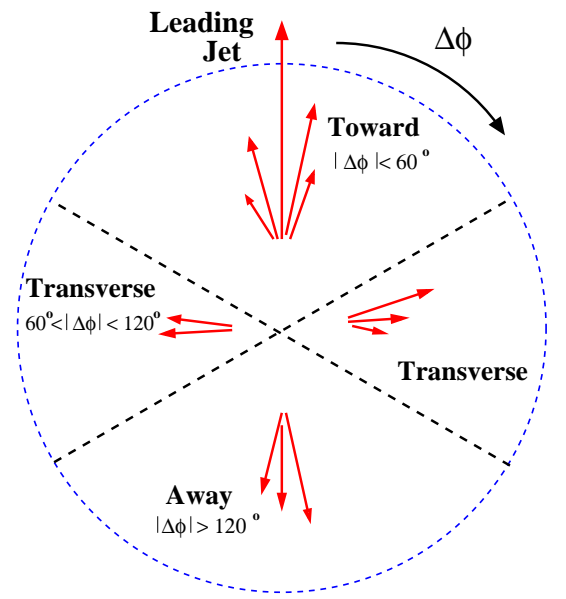

Fig. 16. Event regions defined in terms of the azimuthal angle between charged particles and the leading charged jet, $\Delta \phi=$ $\phi_{\text {particle }}-\phi_{\text {ljet }}$.

Figure 17 shows the average charged particle multiplicity (fig. 17(a)) and $\mathrm{p}_{t}$ sum (fig. 17(b) ) in the transverse region generated with PYTHIA6.214 - $\operatorname{MSTP}(82)=4$ distributions for different values of $\operatorname{PARP}(82)$, i.e. different $\mathrm{p}_{t_{m i n}}$, compared to the data.

Increasing $\mathrm{p}_{t_{\min }}$, which corresponds to a decrease in the rate of semi-hard parton scatterings, results in both $\left\langle N_{\text {chg }}\right\rangle$ and $\left\langle\mathrm{p}_{t_{\text {sum }}}\right\rangle$ decreasing, as seen in figs. 17(a) and (b). This effect is similar to the one observed in fig. 11 for minimum bias charged particle density distributions $\mathrm{dN}_{c h} / \mathrm{d} \eta$.

A noticeable feature in the distributions generated with $\operatorname{PARP}(82)=1.5$ and 2.0 is the irregular shape of the plateau which is not as flat as in the CDF distribution for transverse $\left\langle N_{c h g}\right\rangle$ nor follows the slow rise in $\left\langle\mathrm{p}_{t_{\text {sum }}}\right\rangle$. It shows the presence of a bump for leading jets with $5 \mathrm{GeV}<$ $\mathrm{P}_{t_{l j e t}}<20 \mathrm{GeV}$. The underlying event associated to these low- $\mathrm{p}_{t}$ leading jets is dominated by particles produced in soft interactions which are particularly enhanced by the lower values of $\operatorname{PARP}(82)$.

For events with $\mathrm{P}_{t_{l j e t}}>20 \mathrm{GeV}$, a rise in both $\left\langle N_{c h g}\right\rangle$ and $\left\langle\mathrm{p}_{t_{\text {sum }}}\right\rangle$ is also observed when lower PARP (82) values are used in the event generation. Though smaller than the rise seen for events with low- $\mathrm{p}_{t}$ leading jets, in the region of $\mathrm{P}_{t_{l j e t}}>20 \mathrm{GeV}$ the rise of $\left\langle N_{c h g}\right\rangle$ and $\left\langle\mathrm{p}_{t_{\text {sum }}}\right\rangle$ is more sensitive to the hard component of the underlying event which stems from initial and final state radiation and from a secondary hard scattering falling into the transverse region [20].

Thus lowering $\mathrm{p}_{t_{m i n}}$, the rate of multiple parton interactions increases causing the multiplicity and $\mathrm{p}_{t_{\text {sum }}}$ in the underlying event to rise. However the rise is more accentuated in softer than in harder parton interactions which leads to the change in the shape of the distributions seen in fig. 17 .

An interesting effect in the underlying event is observed for the double Gaussian with different core sizes as shown in fig. 18. It shows the average charged particle multiplicity (fig. 18(a) ) and average $\mathrm{p}_{t}$ sum (fig.

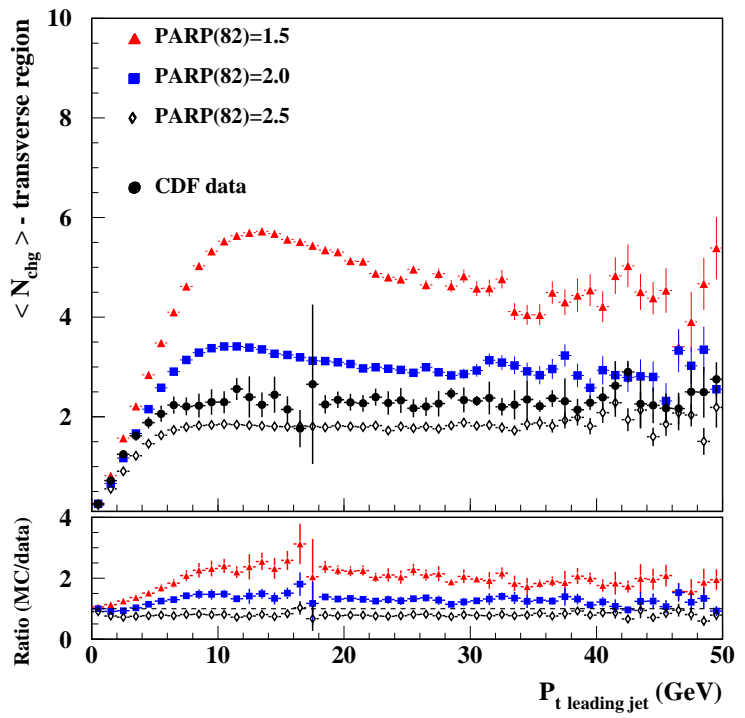

(a)

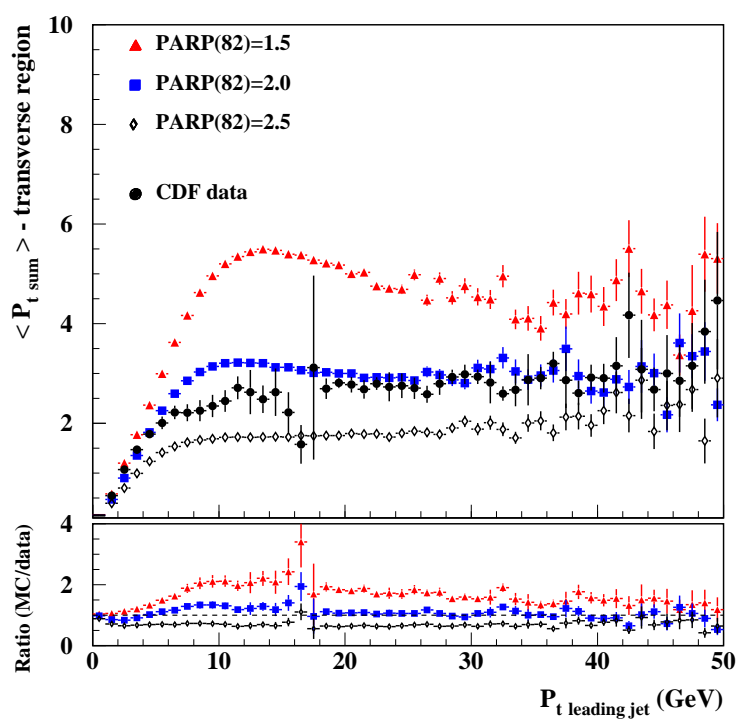

(b)

Fig. 17. (a) Average charged particles multiplicity in the transverse region and (b) average $\mathrm{p}_{t}$ sum in the transverse region varying $\operatorname{PARP}(82)$.

18(b) ) in the transverse region, comparing PYTHIA6.214 - $\operatorname{MSTP}(82)=4$ with different core sizes to the data. $\mathrm{p}_{t_{m i n}}$ is set to the default value in all cases. For example, changing PARP(84) from 0.2 to 0.5 reduces the plateau of $\left\langle N_{c h g}\right\rangle$ by nearly a factor of two, while a further increase in PARP $(84)$ from 0.5 to 0.8 only reduces the plateau by $\sim 15 \%$. In terms of $\chi^{2} /$ d.o.f., comparing PYTHIA 6.214 - $\operatorname{MSTP}(82)=4$ with PARP $(84)=0.2,0.5$ and 0.8 to the data for $\left\langle N_{c h g}\right\rangle$ one gets $\chi^{2} / 50$ d.o.f. $=16.7,0.7$ and 2.2 respectively.

The explanation for the changes in the underlying event due to different core sizes is the same as already discussed for minimum bias events. Jets are likely to be produced 


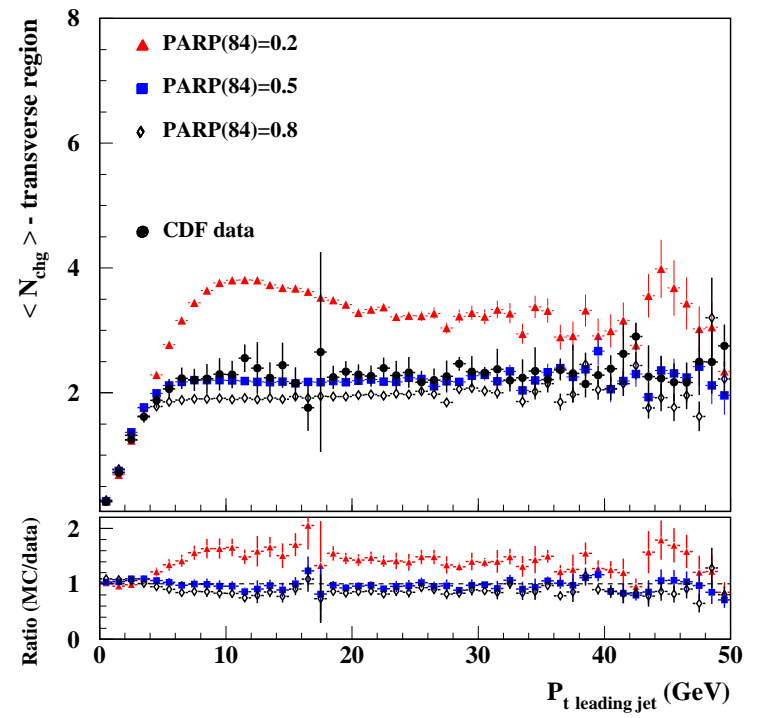

(a)

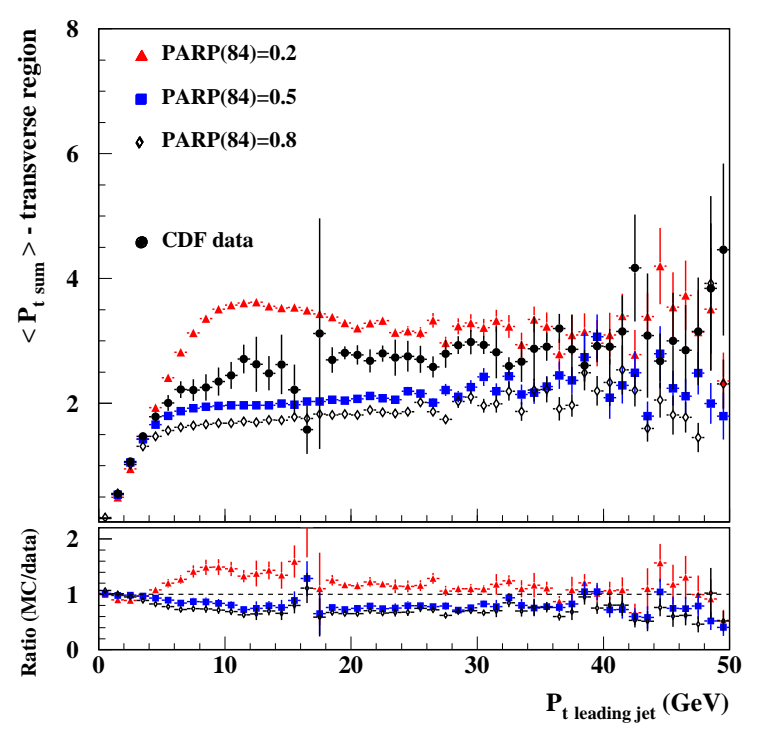

(b)

Fig. 18. (a) Average charged particles multiplicity in the transverse region and (b) average $\mathrm{p}_{t}$ sum in the transverse region varying $\mathrm{PARP}(84)$.

when there is a core overlap in the hadronic collision. Smaller and dense cores imply that events with a core overlap have also a large overlap of less dense matter regions which surround the core, and when overlapped generate high rates of soft interactions causing the higher plateaus observed in both $\left\langle N_{c h g}\right\rangle$ and $\left\langle\mathrm{p}_{t_{\text {sum }}}\right\rangle$ distributions in fig. 18. Larger cores also imply smaller soft surrounding regions in the colliding hadrons, hence producing lower multiplicity and $\mathrm{p}_{t_{\text {sum }}}$ distributions in the underlying event.

If increased to its maximum, the core radius in the double Gaussian matter distribution will actually become the hadronic radius, reproducing the single Gaussian distribution. There is therefore a saturation point in the effect obtained by increasing (or reducing) the core size which can be seen in fig. 18 when the variation in the underlying event for PARP(84) going from 0.2 to 0.5 is larger than the variation caused by changing $\operatorname{PARP}(84)$ from 0.5 to 0.8 .

\subsection{Describing the UE with PHOJET}

As shown in the previous section, PHOJET1.12 with its default options gives a fairly good description of the charged multiplicity and $\mathrm{dN}_{c h} / \mathrm{d} \eta$ distributions in minimum bias events. Here, we compare PHOJET1.12, again with its default options, to the CDF data for charged jet evolution and the underlying event [20].

Figure 19 shows PHOJET1.12 predictions compared to data for: (a) average multiplicity in the transverse region and (b) average $\mathrm{p}_{t_{\mathrm{sum}}}$ in the transverse region.

The MC predictions agree reasonably well to the data for the UE multiplicity distribution, as shown in fig. 19 (a). The $\chi^{2} /$ d.o.f. for the comparisons shown in figs. 19 (a) is $\chi^{2} / 50$ d.o.f. $=5.3$.

Although PHOJET1.12 predicts a multiplicity distribution for the underlying event which agrees fairly well with the data, the same cannot be said for the average $\mathrm{p}_{t_{\text {sum }}}$ distribution (fig. 19(d)). The measured $\left\langle\mathrm{p}_{t_{\text {sum }}}\right\rangle$ distribution is underestimated by PHOJET1.12 by $\sim 20 \%$, and the comparison between $\mathrm{MC}$ and data gives a $\chi^{2} / 50$ d.o.f. of 9.4 .

\subsection{UE vs. minimum bias}

The CDF measurement shows that the underlying event multiplicity forms a plateau for events with $\mathrm{P}_{t_{1 \mathrm{jet}}} \gtrsim 5 \mathrm{GeV}$ at $\left\langle N_{\text {chg }}\right\rangle \sim 2.3$. Supposing that the transverse region in events with $\mathrm{P}_{t_{1 \text { jet }}} \gtrsim 5 \mathrm{GeV}$ is uniform in azimuthal angle $\phi$, for the full $2 \pi$ range this corresponds to 6.9 particles. Assuming also that the transverse region is uniform in pseudorapidity $\eta$, one obtains 3.45 particle per unit pseudorapidity, which in fact, after multiplying by 1.09 to correct for the detector effects, corresponds to $\sim 3.8$ charged particles per unit pseudorapidity with $\mathrm{p}_{t}>0.5 \mathrm{GeV}$. Extrapolating to low- $\mathrm{p}_{t}$ using the form $\mathrm{e}^{-2 p_{t}}[20]$ implies that there are roughly 10 charged particles per pseudorapidity unit with $\mathrm{p}_{t}>0 \mathrm{GeV}$ in the underlying event.

We can now compare the particle density obtained for the plateau in the underlying event distribution to the one shown for minimum bias events in $\mathrm{p} \overline{\mathrm{p}}$ collision at $1.8 \mathrm{TeV}$ in fig. 10. The minimum bias density, which has also been measured by CDF, gives $\mathrm{dN}_{c h} / \mathrm{d} \eta \sim 4$ for $|\eta|<1$ [15], while the equivalent density for the underlying event is at least a factor of two larger. Although this comparison is an approximation due to the uncertainties in estimating the particle density for the underlying event (i.e. extrapolation to low- $\mathrm{p}_{t}$ and several assumptions made on the particle distribution in $\phi$ and $\eta$ ), it clearly shows that the underlying event in hard scattering processes $\left(\mathrm{P}_{t_{1 \mathrm{jet}}} \gtrsim 5\right.$ 


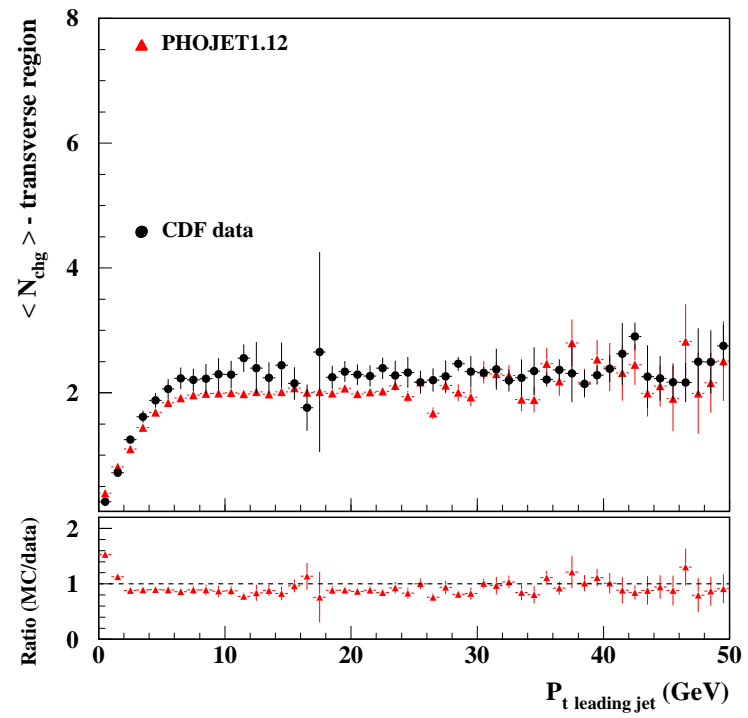

(a)

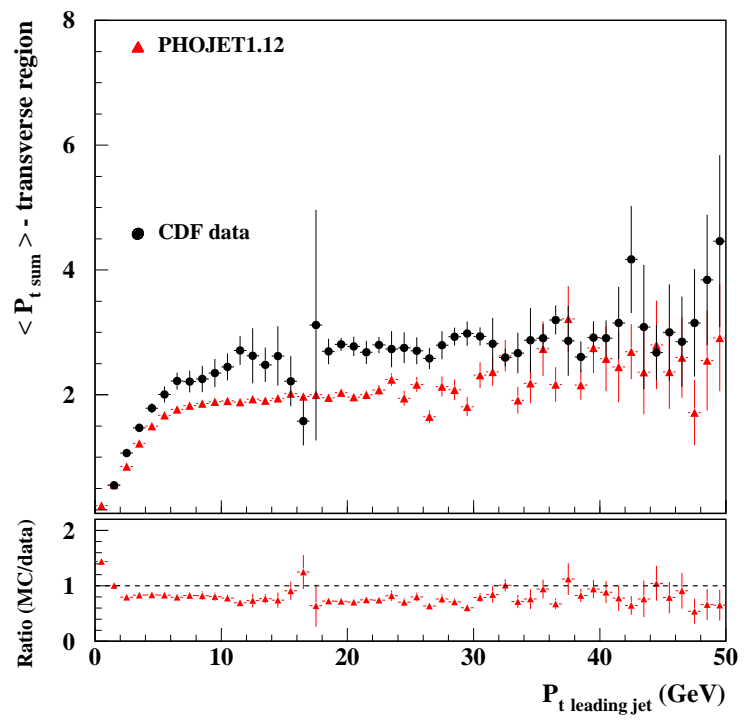

(b)

Fig. 19. PHOJET1.12 predictions compared to CDF data for: (a) average multiplicity in the transverse region and (b) average $\mathrm{p}_{t_{\text {sum }}}$ in the transverse region.

$\mathrm{GeV}$ ) has much more activity than an average minimum bias event.

\section{Describing minimum bias and the UE}

In the previous two sections, we have compared PYTHIA6.214 and PHOJET1.12 to minimum bias and the underlying event data. The comparisons made show that PHOJET1.12 with its default options is reasonably successful in describing the data while PYTHIA6.214 requires a tuning in order to be able to describe either minimum bias or underlying event measurements.

Based on the knowledge of how variations in key PYTHIA parameters affect minimum bias and underlying event distributions we are able to tune PYTHIA6.214 to better reproduce the data.

In this section we present a PYTHIA6.214 tuning which is based on comparisons to a wide range of experimental distributions for both minimum bias and the underlying event. The data we used to tune PYTHIA6.214 is listed in table 3.

The predictions of our PYTHIA6.214 tuned model will also be compared to PHOJET1.12, to a previous ATLAS tuning which was used to generate minimum bias distributions for the ATLAS-TDR $[32,33]$, and to the PYTHIA tuning proposed by the CDF Collaboration [34].

\subsection{PYTHIA6.214 Tuned Model}

PYTHIA6.214 allows the user to select a variety of models to generate soft particle interactions. Based on results of the comparisons presented previously, we opted for the complex scenario with a double Gaussian matter distribution - $\operatorname{MSTP}(82)=4$ - which proved to be a model capable of describing complex features in particle production, particularly the high-multiplicity tails in charged multiplicity distributions.

A tuned set of parameters which allows PYTHIA6.214 - $\operatorname{MSTP}(82)=4$ to reproduce the data can be achieved by regulating the event activity generated by multiple parton scatterings. Combining the effects of variations in $\mathrm{p}_{t_{\min }}$ and in the core-size we obtained a set of PYTHIA6.214 parameters which considerably improves PYTHIA's description of minimum bias and underlying event distributions. Our PYTHIA6.214 tuned parameters are displayed in table 4.

The fundamental difference between our tuned set of parameters and PYTHIA6.214 default settings is our choice for the more sophisticated model of hadron interactions switched on by $\operatorname{MSTP}(82)=4$.

Another significant difference is the increase of the core-radius from the default setting of $20 \%$ of the hadron radius (PARP $(84)=0.2)$ to a larger core of $50 \%$ of the total hadron radius $(\operatorname{PARP}(84)=0.5)$. We have also changed $\mathrm{p}_{t_{\min }}$ slightly by reducing the parameter PARP(82) from its original 1.9 to 1.8 .

To compare with published data, the $\pi^{0}, K_{s}$ and $\Lambda^{0}$ decays are suppressed in our PYTHIA6.214 tuned model $[15,19]$. This will also be applied to the PHOJET1.12 distributions, but not to the events generated by other models.

Figures 20, 21 and 22 show predictions generated by PYTHIA6.214 - tuned and default, and PHOJET1.12 compared to various minimum bias and underlying event distributions. The combined $\chi^{2}$ for the minimum bias distributions shown in figs. 20 and $21\left(\chi_{\min -b i a s}^{2} / 307\right.$ d.o.f. $)$ is 


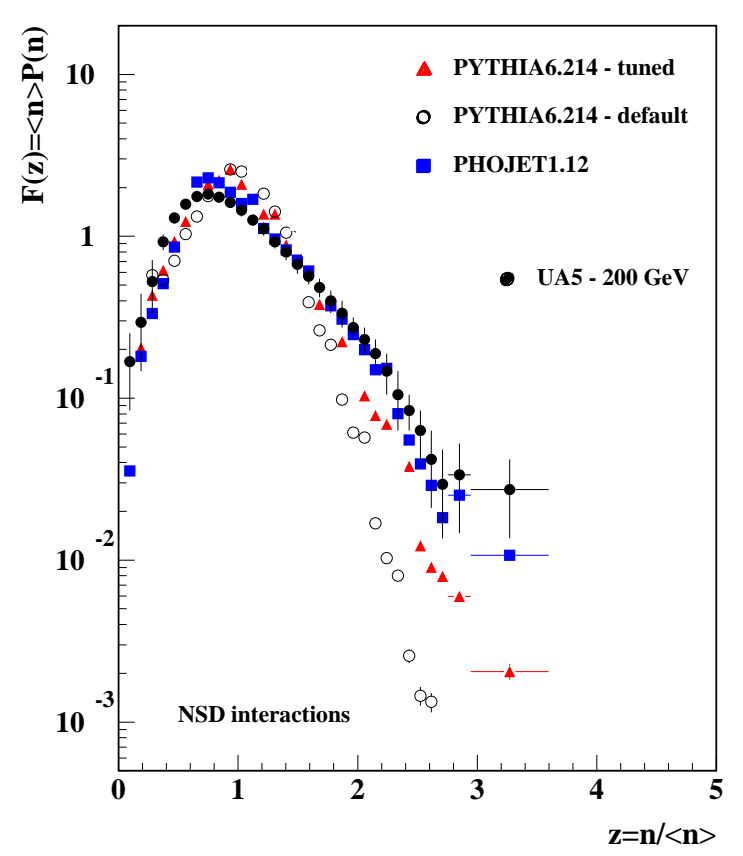

(a)

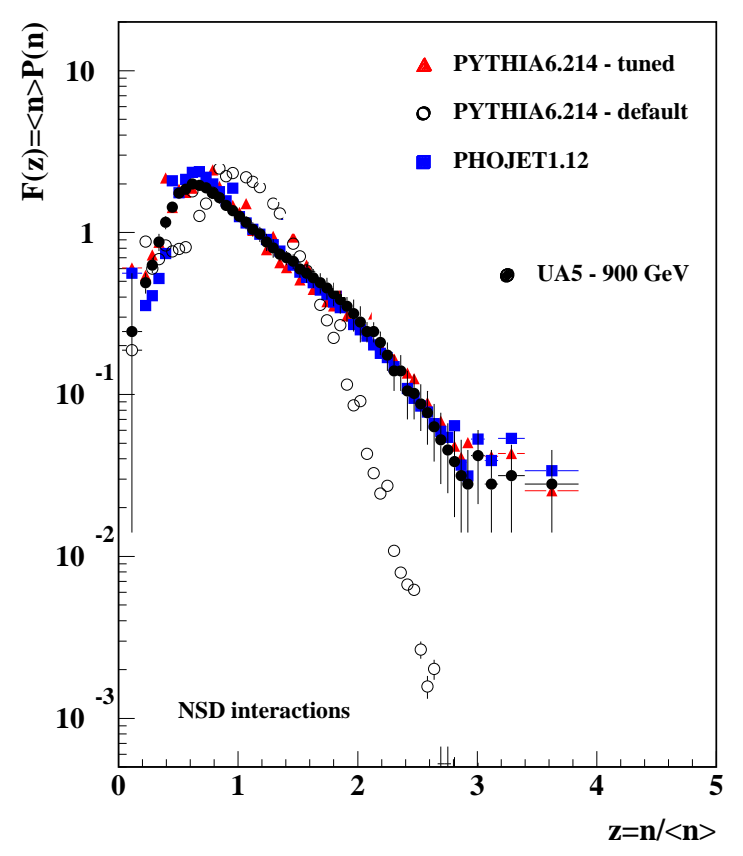

(c)

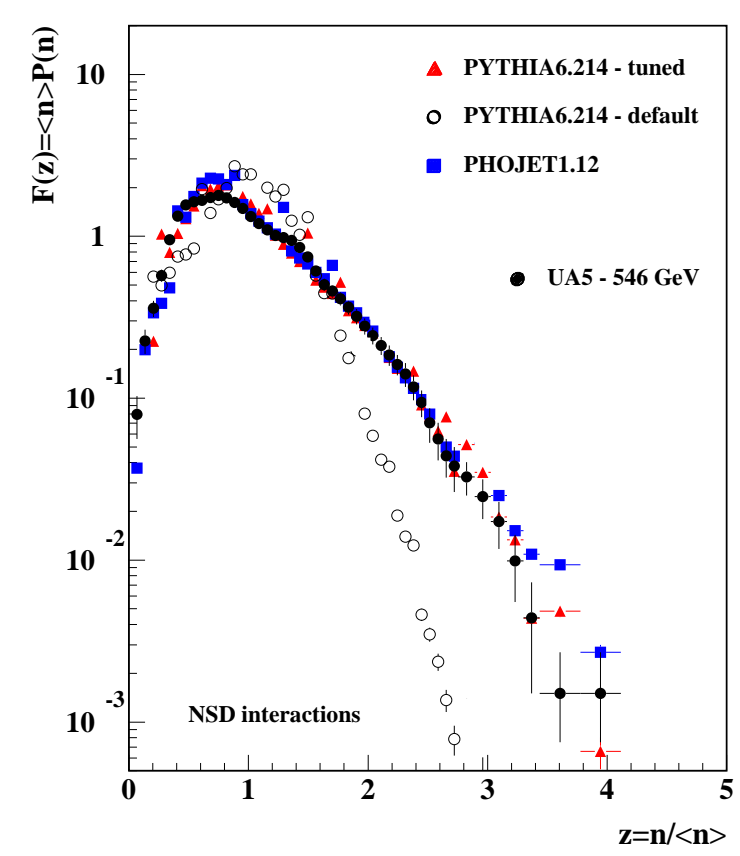

(b)

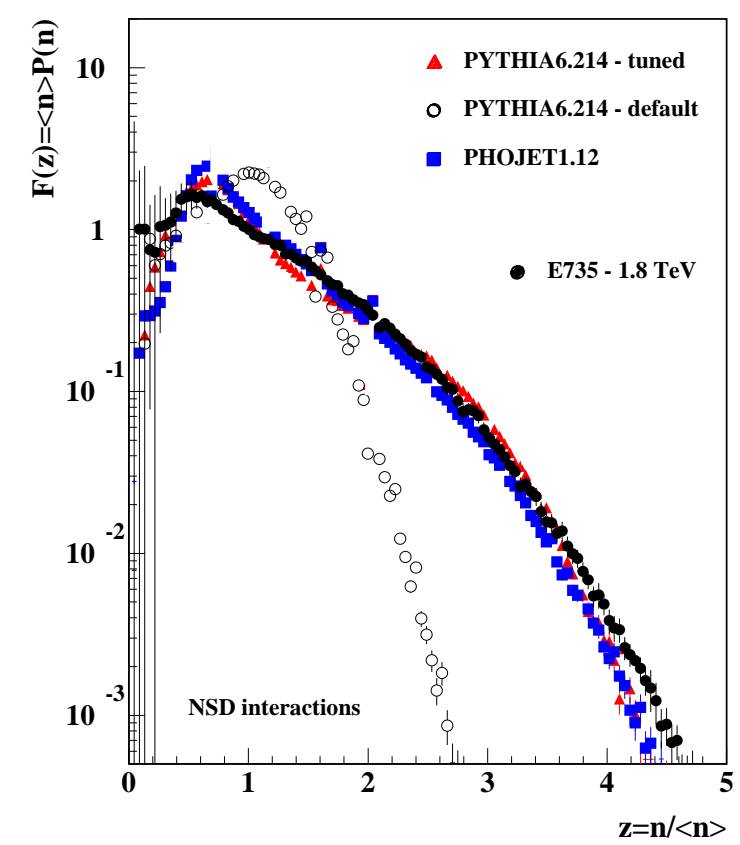

(d)

Fig. 20. Charged multiplicity distributions for NSD p $\bar{p}$ collisions at (a) $\sqrt{\mathrm{s}}=200 \mathrm{GeV}$; (b) $546 \mathrm{GeV}$; (c) $900 \mathrm{GeV}$ and (d) 1.8 TeV. It shows comparisons between PYTHIA6.214 - tuned, PYTHIA6.214 - default, PHOJET1.12 predictions, and the data. 


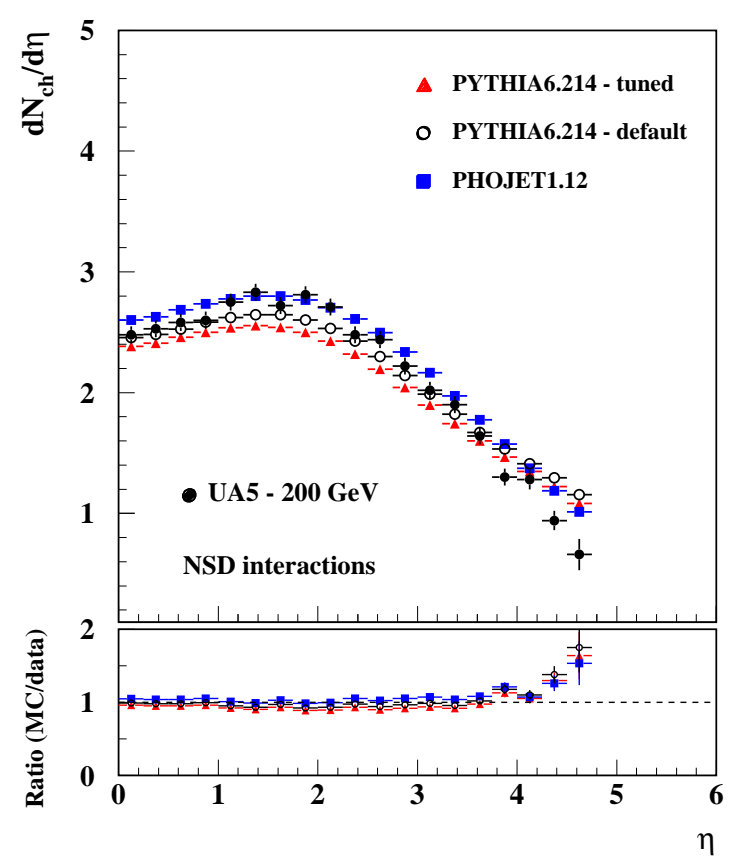

(a)

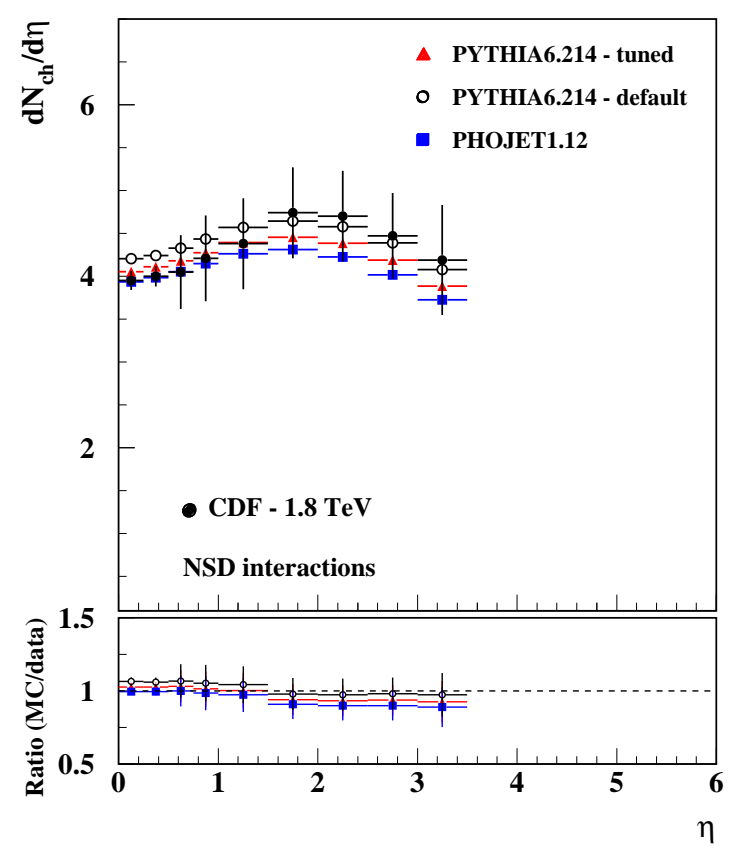

(c)

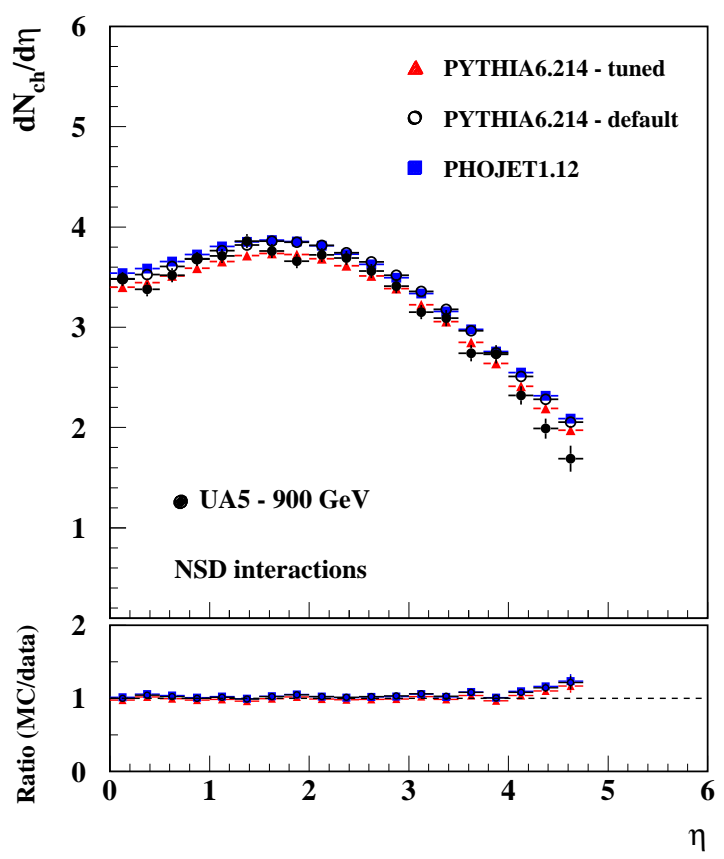

(b)

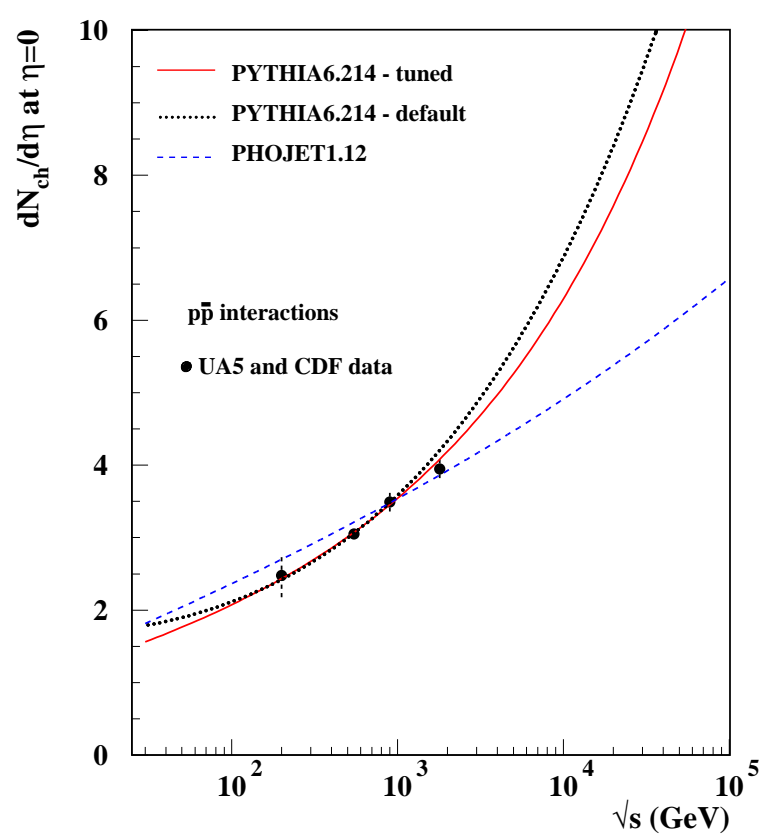

(d)

Fig. 21. Charged particle density distributions, $\mathrm{dN}_{c h} / \mathrm{d} \eta$, for NSD p $\overline{\mathrm{p}}$ collisions at (a) $\sqrt{\mathrm{s}}=200 \mathrm{GeV}$; (b) $900 \mathrm{GeV}$ and (c) 1.8 $\mathrm{TeV}$. In (d) $\mathrm{dN}_{c h} / \mathrm{d} \eta$ at $\eta=0$ for a wide range of $\sqrt{\mathrm{s}}$ is shown. 


\begin{tabular}{|c|c|c|}
\hline Experiment & Colliding beams & Comments \\
\hline CERN - ISR [13] & $\begin{array}{l}\text { pp at } \sqrt{s}=30.4,44.5,52.6 \\
\quad \text { and } 62.2 \mathrm{GeV}\end{array}$ & $\begin{array}{l}\text { charged mult. distributions, }\left\langle n_{c h}\right\rangle \\
\text { and }\left\langle\mathrm{p}_{t}\right\rangle \text { at } \eta=0\end{array}$ \\
\hline UA5 - SPS $[14,18,19]$ & $\mathrm{p} \overline{\mathrm{p}}$ at $\sqrt{s}=200,546$ and $900 \mathrm{GeV}$ & $\begin{array}{c}\mathrm{dN}_{c h} / \mathrm{d} \eta \text { and charged mult. distributions, } \\
\left\langle n_{c h}\right\rangle, \mathrm{dN}_{c h} / \mathrm{d} \eta \text { and }\left\langle\mathrm{p}_{t}\right\rangle \text { at } \eta=0\end{array}$ \\
\hline E735 - Tevatron $[16]$ & $\mathrm{p} \overline{\mathrm{p}}$ at $\sqrt{s}=1.8 \mathrm{TeV}$ & charged mult. distributions \\
\hline CDF - Tevatron $[15,20]$ & $\mathrm{p} \overline{\mathrm{p}}$ at $\sqrt{s}=1.8 \mathrm{TeV}$ & $\begin{array}{l}\quad \mathrm{dN}_{c h} / \mathrm{d} \eta \text { distribution, } \\
\left\langle n_{c h}\right\rangle, \mathrm{dN}_{c h} / \mathrm{d} \eta \text { and }\left\langle\mathrm{p}_{t}\right\rangle \text { at } \eta=0, \\
\text { and }\left\langle N_{c h}\right\rangle \text { and }\left\langle\mathrm{p}_{t_{\text {sum }}}\right\rangle \text { in the UE. }\end{array}$ \\
\hline
\end{tabular}

Table 3. Minimum bias [13-16,18,19] and underlying event data [20] used to tune PYTHIA6.214.

\begin{tabular}{|c|c|}
\hline \multicolumn{2}{|c|}{ PYTHIA6.214 - tuned } \\
\hline 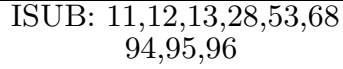 & $\begin{array}{c}\text { QCD } 2 \rightarrow 2 \text { partonic scattering } \\
+ \text { non-diffractive }+ \text { double diffractive }\end{array}$ \\
\hline $\operatorname{MSTP}(51)=7$ & CTEQ5L - selected p.d.f. (default) \\
\hline $\operatorname{MSTP}(81)=1$ & multiple interactions (default) \\
\hline $\operatorname{MSTP}(82)=4$ & $\begin{array}{l}\text { complex scenario } \\
+ \text { double Gaussian matter distribution }\end{array}$ \\
\hline $\operatorname{PARP}(82)=1.8$ & $\mathrm{p}_{t_{\min }}$ parameter \\
\hline $\operatorname{PARP}(84)=0.5$ & $\begin{array}{l}\text { core radius: } 50 \% \text { of the } \\
\text { hadronic radius }\end{array}$ \\
\hline $\operatorname{PARP}(89)=1.0$ & $\begin{array}{l}\text { energy scale }(\mathrm{TeV}) \text { used to } \\
\text { calculate } \mathrm{p}_{t_{\min }} \text { (default) }\end{array}$ \\
\hline $\operatorname{PARP}(90)=0.16$ & $\begin{array}{l}\text { power of the energy dependence } \\
\text { of } \mathrm{p}_{t_{\min }} \text { (default) }\end{array}$ \\
\hline
\end{tabular}

Table 4. PYTHIA6.214 tuned parameters for minimum bias and the underlying event.

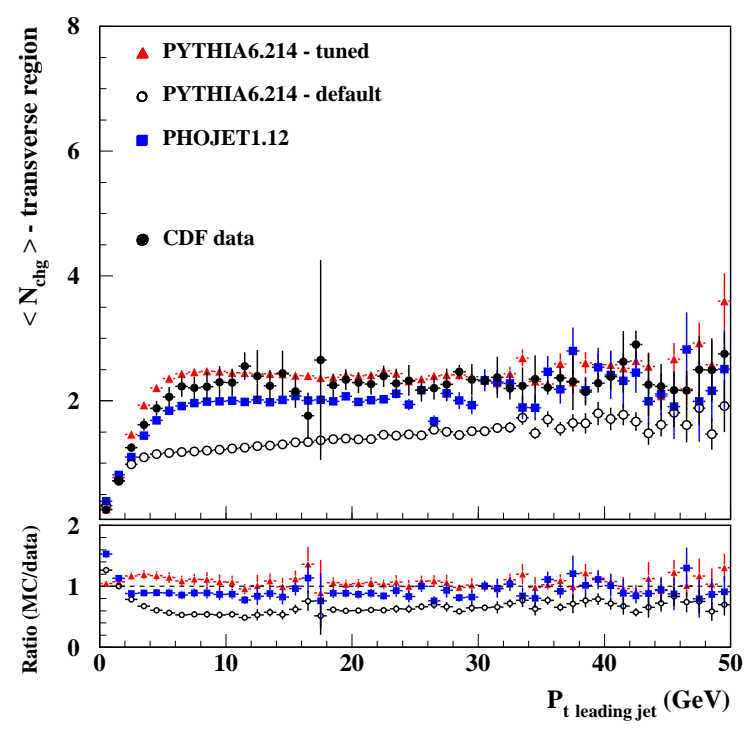

(a)

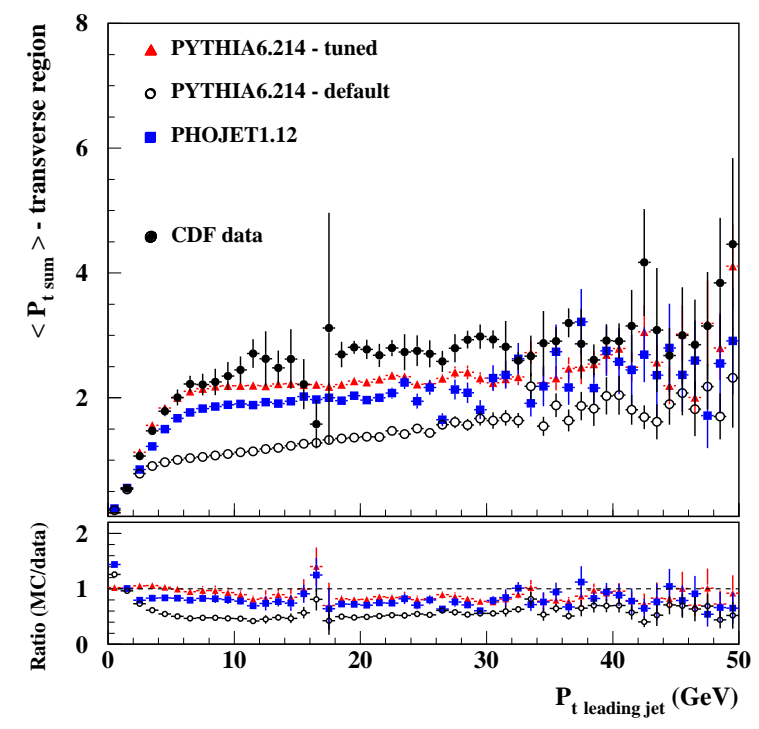

(b)

Fig. 22. PYTHIA6.214 - tuned and default, and PHOJET1.12 predictions compared to CDF data for: (a) average multiplicity in the underlying event and (b) average $\mathrm{p}_{t_{\text {sum }}}$ in the underlying event. 
68.0, 11.6 and 8.2 for PYTHIA6.214 - default, PYTHIA6.214 - tuned and PHOJET1.12, respectively. Similarly, for the underlying event distributions displayed in figs. $22(\mathrm{a})$ and (b), the combined $\chi^{2}\left(\chi_{\mathrm{UE}}^{2} / 100\right.$ d.o.f. $)$ is 22.7 , 2.1 and 7.4, again for PYTHIA6.214 - default and tuned, and PHOJET1.12, respectively.

The description of both minimum bias and underlying event distributions is clearly improved by using PYTHIA6.214 - tuned compared to the predictions generated by the default settings. As already indicated in comparisons presented in previous sections, PHOJET1.12 is also considerably more accurate in describing the data than PYTHIA6.214 - default.

The distributions in figs. 20, 21 and 22 and the combined $\chi^{2}$ derived from both minimum bias and underlying event distributions show that PYTHIA6.214 - tuned and PHOJET1.12 are compatible to the data. With $\chi_{\text {min-bias }}^{2}=$ 8.2, PHOJET1.12 generates slightly better predictions for minimum bias event distributions than PYTHIA6.214 tuned, which has $\chi_{\text {min-bias }}^{2}=11.6$. The opposite happens for underlying event distributions which are better described by PYTHIA6.214 - tuned $\left(\chi_{\mathrm{UE}}^{2}=2.1\right)$ than by PHOJET1.12 $\left(\chi_{\mathrm{UE}}^{2}=7.4\right)$. The global $\chi^{2}$, combining 307 minimum bias degrees of freedom and 100 from the underlying event distributions, is $\chi_{\text {global }}^{2} / 407$ d.o.f. $=7.9$ for PHOJET1.12 and $\chi_{\text {global }}^{2} / 407$ d.o.f. $=9.3$ for PYTHIA6.214 - tuned. PYTHIA6.214 - default compared to the data gives $\chi_{\text {global }}^{2} / 407$ d.o.f. $=56.9$.

\subsection{Alternative PYTHIA Tunings}

In this section we compare our PYTHIA6.214 tuned model to two other PYTHIA tunings for soft hadronic interactions, namely, the ATLAS - TDR model used to generate minimum bias events and the underlying structure of jet analysis at ATLAS [32] and the tuning proposed by the CDF Collaboration [34].

The ATLAS Technical Design Report (ATLAS-TDR) shows minimum bias predictions for the LHC which have been based on an early study of minimum bias MC models presented in [33]. The parameters used in the generation of minimum bias events for the ATLAS-TDR are shown in table 6 . These parameters were obtained as a result of comparisons between PYTHIA5.724 and UA5 and CDF minimum bias data for $\mathrm{dN}_{c h} / \mathrm{d} \eta$ and CDF measurements for one-particle inclusive $\mathrm{p}_{t}$ spectra in the central rapidity region. Though [33] has not used underlying event distributions to tune the MC model for soft particle production, the PYTHIA parameters in table 6 were also used to generate the underlying event in jet studies throughout the ATLAS-TDR.

The PYTHIA parameters proposed as the CDF tuning (also known as "PYTHIA tune A") are shown in table 7 [34]. This tuning was obtained by comparing PYTHIA6.206 to minimum bias $\mathrm{dN}_{c h} / \mathrm{d} \eta$ distributions (at $\sqrt{\mathrm{s}}=630 \mathrm{GeV}$ and $1.8 \mathrm{TeV}$ ) and underlying event data (at $\sqrt{\mathrm{s}} 1.8 \mathrm{TeV}$ ) [20] measured by the CDF collaboration. One of the interesting aspects of the CDF tuning, is that $\operatorname{PARP}(67)$ [12] is changed from its default value of 1 to 4 , effectively increasing the maximum parton virtuality allowed in parton showers. This is done to explain the $\mathrm{p}_{t}$ spectrum of particles in the underlying event. This was not done for the current study, but will be looked at in future.

Figures 23, 24 and 25 compare PYTHIA predictions generated with PYTHIA6.214 - tuned, ATLAS - TDR and CDF tuning parameters to data for both minimum bias and the underlying event. The combined $\chi_{\text {min-bias }}^{2}$ for the distributions shown in figs. 23 and 24 is 38.9 and 26.7 for the ATLAS - TDR and CDF tuning models, respectively. Similarly, for the underlying event distributions displayed in fig. 25, the combined $\chi_{\mathrm{UE}}^{2}$ is 15.0 and 1.3 , again for the distributions generated with the ATLAS - TDR and the CDF tuning, respectively.

The $\chi_{\text {min-bias }}^{2}$ from both ATLAS - TDR and CDF tuning models are worse than the $\chi_{\text {min-bias }}^{2}=11.6$ obtained for PYTHIA6.214 - tuned. This reflects the fact that we have used a wider set of experimental distributions than the other tunings to guide our choice of parameters. In particular, we have used charged multiplicity distributions measured at different colliding energies to better understand the effects of multiple parton interactions, which the other models have not.

Although the minimum bias data shown in figs. 23 and 24 represents non-single diffractive inelastic interactions (i.e. non-diffractive inelastic and double diffractive events), the ATLAS - TDR model does not include double diffraction in its description of minimum bias events. This is a particular disadvantage of this model in its attempt to describe minimum bias events as defined in this work, and source of considerable disagreements between the model predictions and the data for low-multiplicity events $(\mathrm{z}<1)$ as can be seen in fig. 23. The CDF tuning was obtained by simulating the CDF "min-bias" trigger requirements, which picks up some double diffractive events. The subprocess 94 is therefore included in table 7 to give a good approximation of the CDF's trigger requirements for minimum bias events selection.

Comparing the underlying event distributions generated with ATLAS - TDR, CDF tuning and our PYTHIA 6.214 - tuned parameters, the best $\chi_{\mathrm{UE}}^{2}$ is obtained with the CDF tuning parameters. On the other hand, predictions generated with the ATLAS - TDR parameters considerably overestimate the data with $\chi_{\mathrm{UE}}^{2}=15.0$.

There are two fundamental problems limiting the agreement between the ATLAS - TDR distributions and the data and its predictive power for both minimum bias and the underlying event: the hadronic core size and the $\mathrm{p}_{t_{\min }}$ used in this model. Firstly, with a core radius corresponding to $20 \%$ of the hadron's radius, too much event activity accompanies the hard partonic scatterings causing it to overestimate the underlying event multiplicity and average $\mathrm{p}_{t_{\text {sum }}}$ as well as overestimating most of the highmultiplicity tails of charged multiplicity distributions. The second problem is the absence of an energy dependence correction for $\mathrm{p}_{t_{\min }}$, this was introduced for PYTHIA versions later than PYTHIA6.1 [12]. 


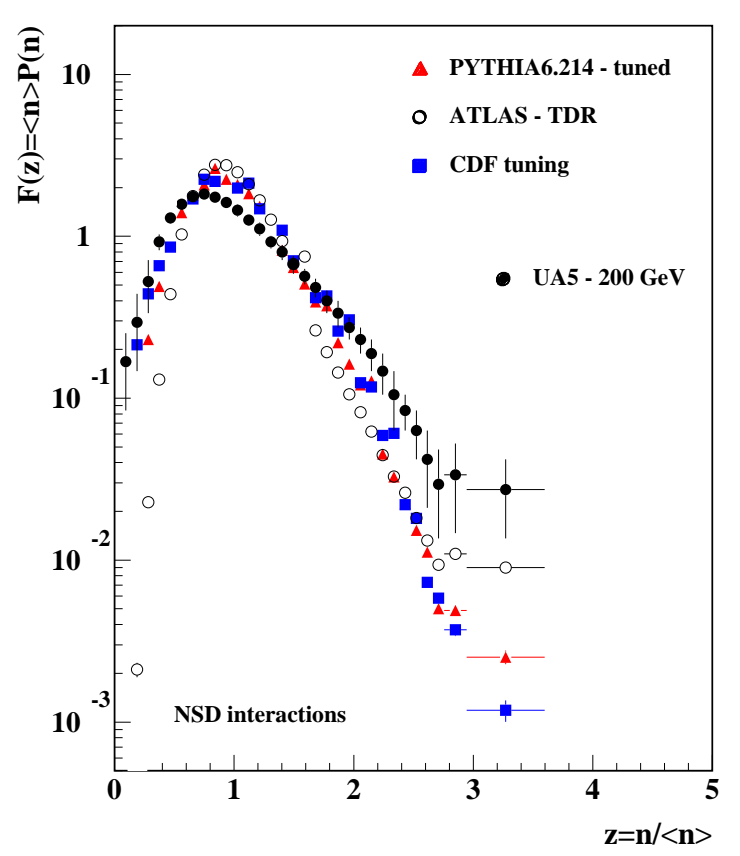

(a)

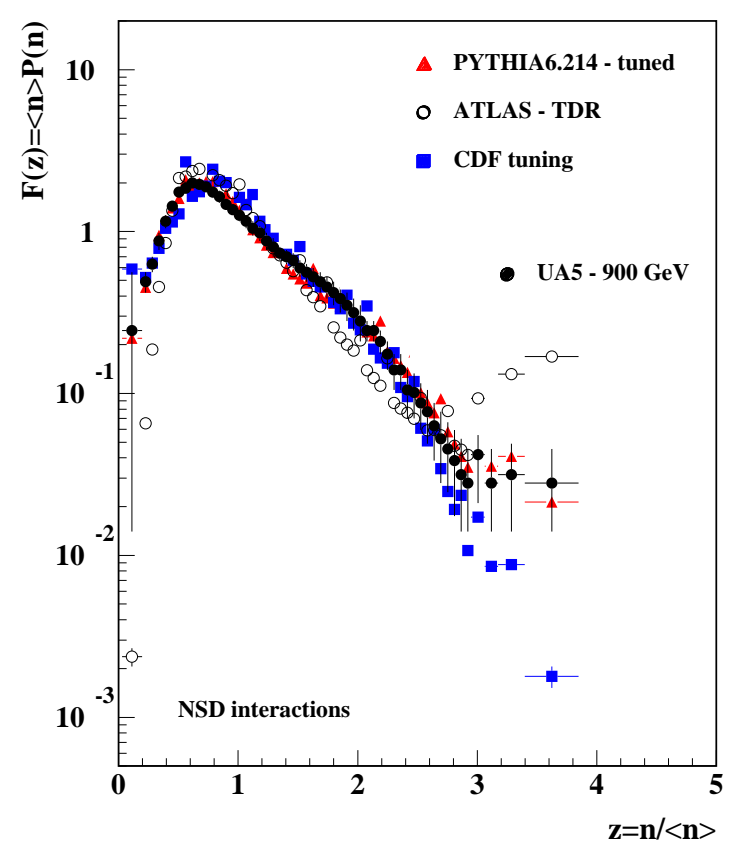

(c)

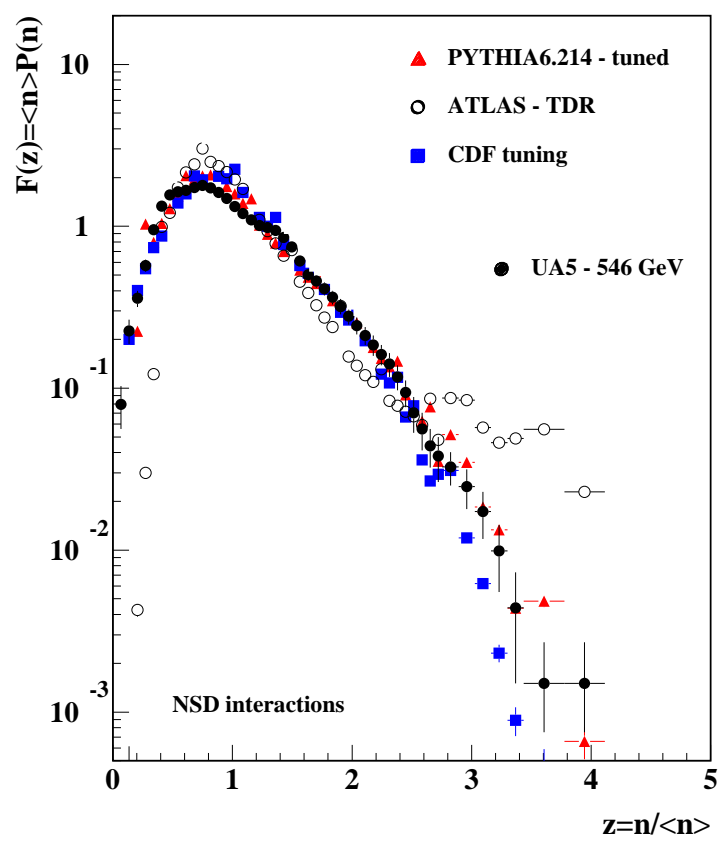

(b)

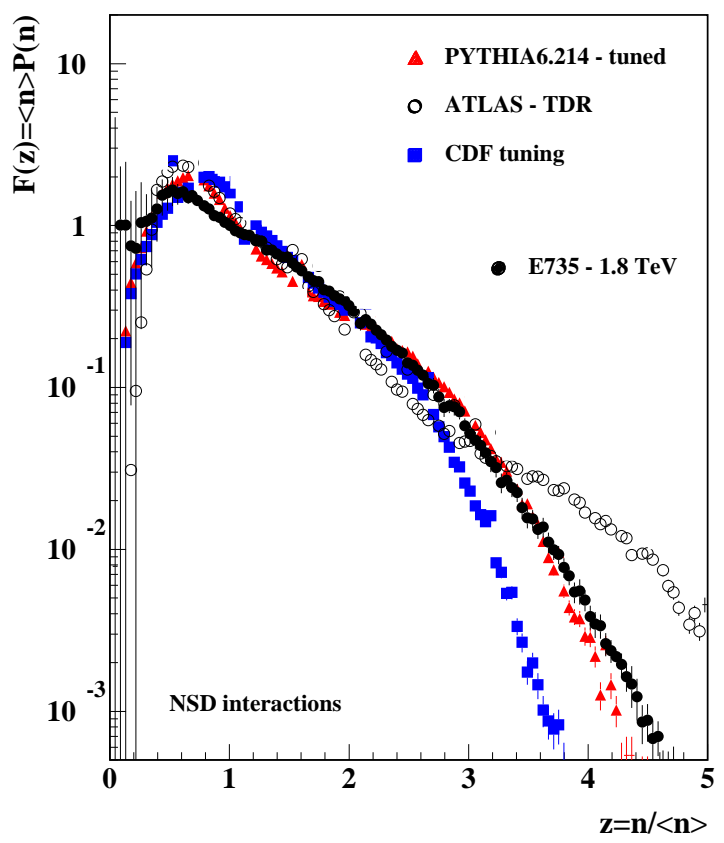

(d)

Fig. 23. Charged multiplicity distributions for NSD p $\bar{p}$ collisions at (a) $\sqrt{\mathrm{s}}=200 \mathrm{GeV}$; (b) $546 \mathrm{GeV}$; (c) $900 \mathrm{GeV}$ and (d) 1.8 TeV. It shows comparisons between PYTHIA predictions generated with our tuned model, ATLAS - TDR parameters and the CDF tuning to the data. 


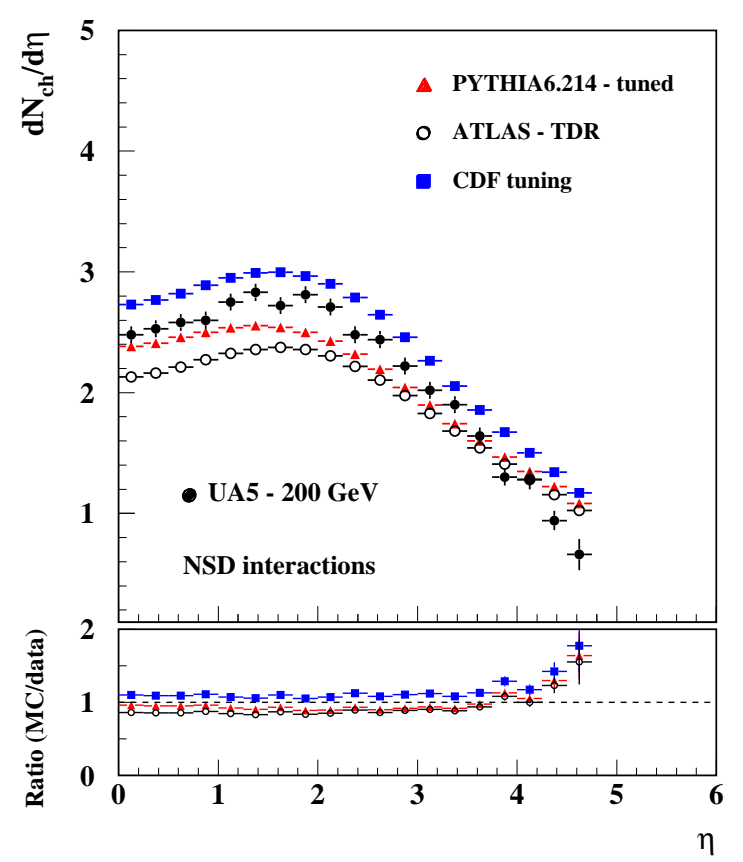

(a)

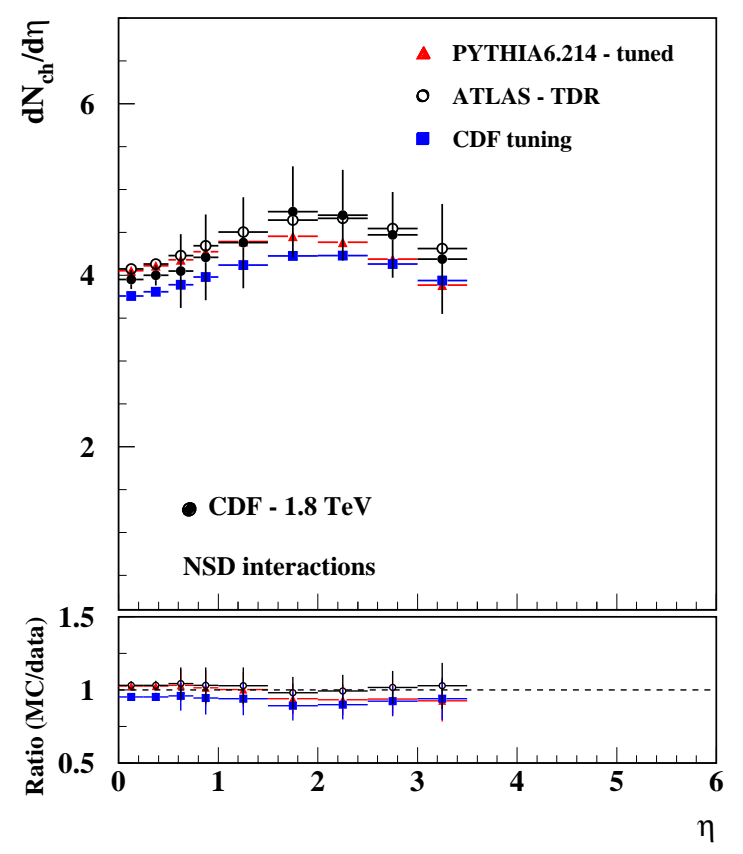

(c)

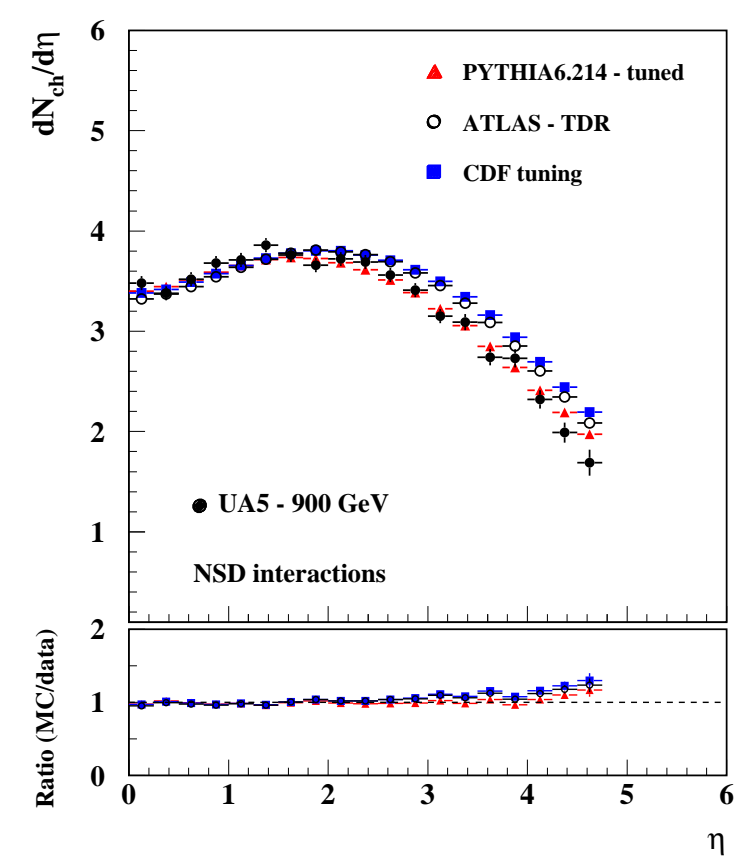

(b)

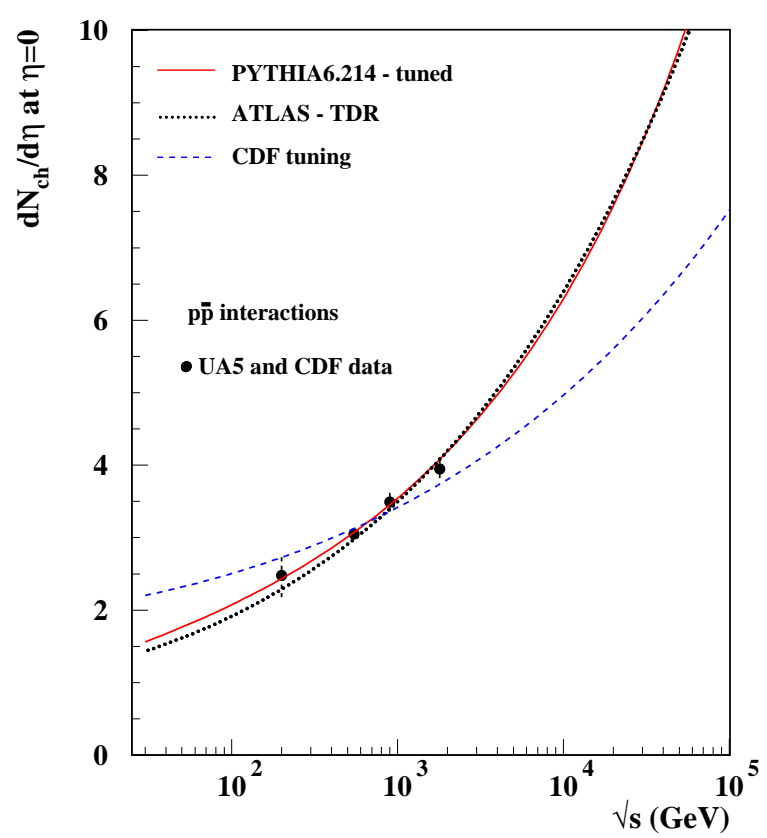

(d)

Fig. 24. Charged particle density distributions, $\mathrm{dN}_{c h} / \mathrm{d} \eta$, for NSD p $\overline{\mathrm{p}}$ collisions at (a) $\sqrt{\mathrm{s}}=200 \mathrm{GeV}$; (b) $900 \mathrm{GeV}$ and (c) 1.8 $\mathrm{TeV}$. In $(\mathrm{d}) \mathrm{dN}_{c h} / \mathrm{d} \eta$ at $\eta=0$ for a wide range of $\sqrt{\mathrm{s}}$ is shown. MC distributions were generated with PYTHIA6.214 - tuned, ATLAS - TDR and CDF tuning parameters. 


\begin{tabular}{|c|c|c|c|}
\cline { 2 - 4 } \multicolumn{1}{c|}{} & $\begin{array}{c}\text { PYTHIA6.214 - } \\
\text { tuned }\end{array}$ & $\begin{array}{c}\text { PYTHIA6.214 - } \\
\text { default }\end{array}$ & PHOJET1.12 \\
\hline $\begin{array}{c}\text { Charged multiplicity } \\
\text { distributions (fig. } 20) \\
\chi^{2} / 260 \text { d.o.f } \\
\left.\mathrm{dN}_{\text {ch }} / \mathrm{d} \eta \text { (fig. } 21\right) \\
\chi^{2} / 47 \text { d.o.f }\end{array}$ & 13.1 & 79.7 & 9.1 \\
$\begin{array}{c}\left\langle N_{\text {chg }}\right\rangle \text { in the UE } \\
\text { (fig. 22(a)) } \chi^{2} / 50 \text { d.o.f }\end{array}$ & 3.6 & 3.3 & 5.9 \\
$\begin{array}{c}\left\langle\mathrm{p}_{\text {tsum }}\right\rangle \text { in the UE } \\
\text { (fig. 22(b)) } \chi^{2} / 50 \text { d.o.f }\end{array}$ & 1.3 & 15.7 & 9.4 \\
\hline$\chi_{\text {min-bias }}^{2} / 307$ d.o.f & 11.62 & 29.7 & 8.2 \\
$\chi_{\mathrm{UE}}^{2} / 100$ d.o.f & 2.07 & 68.0 & 7.4 \\
$\chi_{\text {global }}^{2} / 407$ d.o.f & 9.27 & 22.7 & 7.9 \\
\hline \hline
\end{tabular}

Table 5. Table of $\chi^{2} /$ d.o.f. for PYTHIA6.214 - tuned, PYTHIA6.214 - default and PHOJET1.12.

\begin{tabular}{|c|c|}
\hline \multicolumn{2}{|c|}{ ATLAS - TDR Parameters for Minimum Bias } \\
\hline PYTHIA5.724 & PYTHIA version \\
\hline $\begin{array}{c}\text { ISUB: } 11,12,13,28,53,68 \\
95,96\end{array}$ & $\begin{aligned} \text { QCD } 2 & \rightarrow 2 \text { partonic scattering } \\
& + \text { non-diffractive }\end{aligned}$ \\
\hline $\operatorname{MSTP}(51)=9$ & CTEQ2L - selected p.d.f. \\
\hline $\operatorname{MSTP}(81)=1$ & multiple interactions \\
\hline $\operatorname{MSTP}(82)=4$ & $\begin{array}{l}\text { complex scenario } \\
+ \text { double Gaussian matter distrib. }\end{array}$ \\
\hline $\operatorname{MSTP}(2)=2$ & $\begin{array}{l}\text { NLO formula for } \alpha_{s} \\
\text { in } 2 \rightarrow 2 \text { matrix elements }\end{array}$ \\
\hline $\operatorname{MSTP}(33)=3$ & $\begin{array}{c}\text { inclusion of } \mathrm{K} \text { factors } \\
\text { in hard } 2 \rightarrow 2 \text { cross-sections }\end{array}$ \\
\hline $\operatorname{PARP}(82)=1.55$ & $\mathrm{p}_{t_{\min }}$ parameter \\
\hline $\operatorname{PARP}(84)=0.2$ & $\begin{array}{l}\text { core radius: } 20 \% \text { of the } \\
\text { hadronic radius }\end{array}$ \\
\hline
\end{tabular}

Table 6. ATLAS - TDR parameters for minimum bias events.

Combining the minimum bias and underlying event $\chi^{2}$ s, one has $\chi_{\text {global }}^{2}=20.4$ for the predictions generated with the CDF tuning and $\chi_{\text {global }}^{2}=33.1$ for the distributions generated with the ATLAS - TDR parameters. The $\chi_{\text {global }}^{2}$ for the CDF tuning is greater than the $\chi_{\text {global }}^{2}=9.3$ obtained for PYTHIA6.214 - tuned.

\subsection{Summary of MC model comparisons}

Table 9 summarises the $\chi_{\min -b i a s}^{2}, \chi_{\mathrm{UE}}^{2}$ and $\chi_{\text {global }}^{2}$ obtained by comparisons between data and prediction from $\mathrm{PHO}-$ JET1.12, PYTHIA6.214 - tuned and default, CDF tuning and ATLAS - TDR parameters.

The minimum bias and underlying event distributions presented in this article are satisfactorily described by both PHOJET1.12 and PYTHIA6.214 appropriately tuned.

The tuning we propose, PYTHIA6.214 - tuned (table 4 ), is a good example of a successful PYTHIA tuning for predicting minimum bias and the underlying event. It is undoubtedly a significant improvement compared to the predictions generated with the default PYTHIA6.214 parameters and also to the distributions generated with the parameters used in the ATLAS - TDR and in the CDF tuning. The differences between these models exposes the different procedures used to find each particular tuning. For example, the fact that the CDF tuning was primarily derived based on UE data makes it the best model for this particular set of distributions however, it does not necessarily succeed in reproducing the minimum bias data shown here.

Due to the fact that information from a wider range of comparisons to data was used in our tuning we believe it to be a more robust model than any of the other competing PYTHIA tunings discussed in this study. 


\begin{tabular}{|c|c|}
\hline \multicolumn{2}{|c|}{ CDF Tuning for Minimum Bias and the UE } \\
\hline PYTHIA6.206 & PYTHIA version \\
\hline $\begin{array}{c}\text { ISUB: } 11,12,13,28,53,68 \\
(94), 95,96\end{array}$ & $\begin{array}{c}\text { QCD } 2 \rightarrow 2 \text { partonic scattering } \\
+ \text { non-diffractive }+ \text { double diffractive }\end{array}$ \\
\hline $\operatorname{MSTP}(51)=7$ & CTEQ5L - selected p.d.f. \\
\hline $\operatorname{MSTP}(81)=1$ & multiple interactions \\
\hline $\operatorname{MSTP}(82)=4$ & $\begin{array}{c}\text { complex scenario } \\
+ \text { double Gaussian matter distribution }\end{array}$ \\
\hline $\operatorname{PARP}(67)=4$ & $\begin{array}{l}\text { parameter regulating } \\
\text { initial state radiation }\end{array}$ \\
\hline $\operatorname{PARP}(82)=2.0$ & $\mathrm{p}_{t_{\min }}$ parameter \\
\hline $\operatorname{PARP}(84)=0.4$ & $\begin{array}{l}\text { core radius: } 40 \% \text { of the } \\
\text { hadronic radius }\end{array}$ \\
\hline $\operatorname{PARP}(85)=0.9$ & $\begin{array}{l}\text { probability that gluons will be } \\
\text { colour connected to nearest neighbours }\end{array}$ \\
\hline $\operatorname{PARP}(86)=0.95$ & $\begin{array}{l}\text { probability to produce gluons either } \\
\text { as in } \operatorname{PARP}(85) \text { or as a closed gluon loop }\end{array}$ \\
\hline $\operatorname{PARP}(89)=1.8$ & $\begin{array}{l}\text { energy scale }(\mathrm{TeV}) \text { used to } \\
\text { calculate } \mathrm{p}_{t_{\min }}\end{array}$ \\
\hline $\operatorname{PARP}(90)=0.25$ & $\begin{array}{c}\text { power of the energy dependence } \\
\text { of } \mathrm{p}_{t_{\min }}\end{array}$ \\
\hline
\end{tabular}

Table 7. CDF tuning for minimum bias and the underlying event.

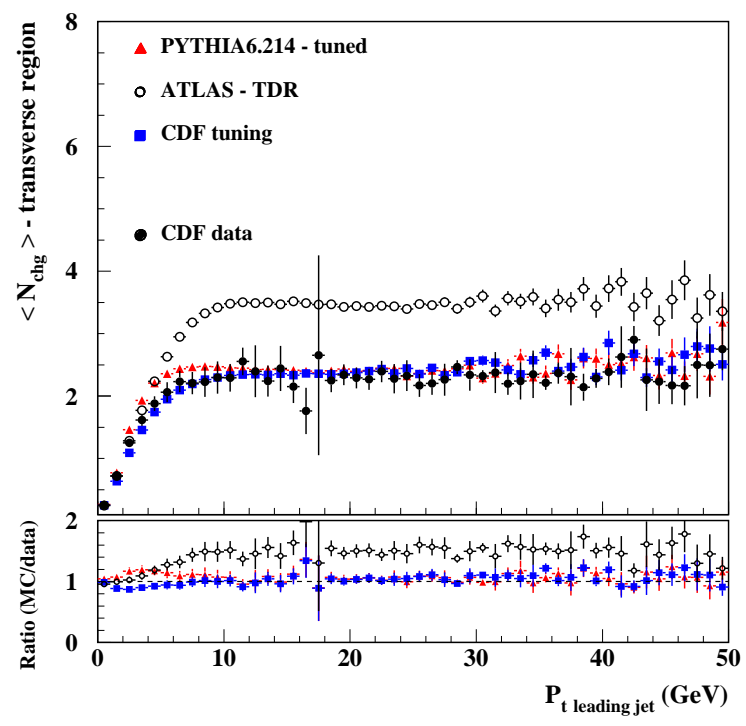

(a)

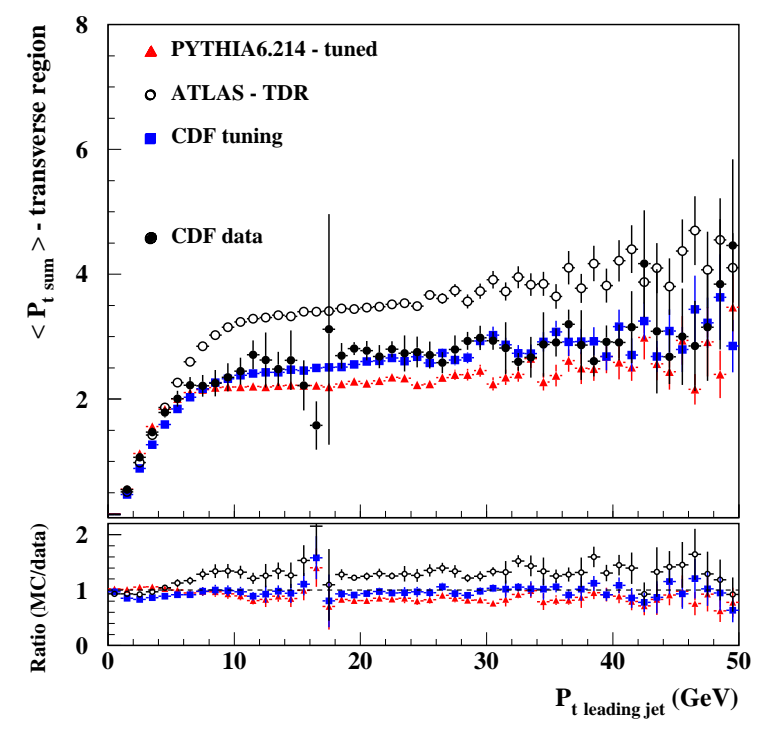

(b)

Fig. 25. PYTHIA6.214 - tuned, ATLAS - TDR and CDF tuning predictions compared to CDF data for: (a) average multiplicity in the underlying event and (b) average $\mathrm{p}_{t_{\text {sum }}}$ in the underlying event. 


\begin{tabular}{|c|c|c|}
\cline { 2 - 3 } \multicolumn{1}{c|}{} & CDF tuning & ATLAS - TDR \\
\hline $\begin{array}{c}\text { Charged multiplicity } \\
\text { distributions (fig. 23) } \\
\chi^{2} / 260 \text { d.o.f } \\
\left.\mathrm{dN}_{\text {ch }} / \mathrm{d} \eta \text { (fig. } 24\right) \\
\chi^{2} / 47 \text { d.o.f } \\
\left\langle N_{\text {chg }}\right\rangle \text { in the UE } \\
\text { (fig. } 25(\mathrm{a})) \chi^{2} / 50 \text { d.o.f } \\
\left\langle\begin{array}{c}\left\langle\mathrm{p}_{t_{\text {sum }}}\right\rangle \text { in the UE } \\
\text { (fig. } 25(\mathrm{~b})) \chi^{2} / 50 \text { d.o.f }\end{array}\right.\end{array}$ & 29.9 & 44.2 \\
\hline$\chi_{\text {min-bias }}^{2} / 307$ d.o.f & 1.6 & 20.6 \\
$\chi_{\mathrm{UE}}^{2} / 100$ d.o.f & 26.7 & 9.5 \\
$\chi_{\text {global }}^{2} / 407$ d.o.f & 20.4 & 38.9 \\
\hline \hline
\end{tabular}

Table 8. Table of $\chi^{2} /$ d.o.f. for the CDF tuning and ATLAS - TDR models.

\begin{tabular}{|c|c|c|c|}
\hline Models & $\chi_{\text {min-bias }}^{2}$ & $\chi_{\mathrm{UE}}^{2}$ & $\chi_{\text {global }}^{2}$ \\
\hline PHOJET1.12 & 8.2 & 7.4 & 7.9 \\
PYTHIA6.214 - tuned & 11.6 & 2.1 & 9.3 \\
CDF tuning & 26.7 & 1.3 & 20.4 \\
ATLAS - TDR & 38.9 & 15.0 & 33.1 \\
PYTHIA6.214 - default & 68.0 & 22.7 & 56.9 \\
\hline
\end{tabular}

Table 9. Table of $\chi_{\text {min-bias }}^{2}, \chi_{\mathrm{UE}}^{2}$ and $\chi_{\text {global }}^{2}$ for PHOJET1.12, PYTHIA6.214 - tuned and default, CDF tuning and ATLAS TDR parameters.

\subsection{Extrapolating predictions to higher energies}

One of the aims of this study is to verify how well minimum bias and the underlying event are reproduced by MC models. Once we identify models which appropriately describe the data, these will then be used to generate predictions for the pp collisions at LHC.

Figure 26 shows the average charged particle multiplicity (a) and the average transverse momentum of charged particles $\left\langle\mathrm{p}_{t}\right\rangle$ at $\eta=0$ (b) for NSD $\mathrm{p} \overline{\mathrm{p}}$ collisions for a wide range of colliding energies. It compares predictions generated with the models which had the best two $\chi_{\text {global }}^{2}$ in the comparisons presented above, i.e. PHOJET1.12 and PYTHIA6.214 - tuned, and the CDF tuning.

The average charged particle multiplicity distributions in fig. 26(a) show small differences between the three model predictions for energies up to the Tevatron $(\sqrt{s}=1.8$ $\mathrm{TeV})$. However, as $\sqrt{s} \rightarrow \infty$ the rise of $\left\langle\mathrm{n}_{c h}\right\rangle$ has a different behaviour for each model: PYTHIA6.214 - tuned gives the steeper rise following a strong $\ln ^{2}(\mathrm{~s})$ dependence, while PHOJET1.12 predicts the slower rise with a dominating $\ln (\mathrm{s})$ behaviour and the CDF tuning distribution sits in between the other two predictions. At the LHC energy, $\sqrt{s}=14 \mathrm{TeV}$, the models predict $70 \lesssim\left\langle\mathrm{n}_{c h}\right\rangle \lesssim 95$.

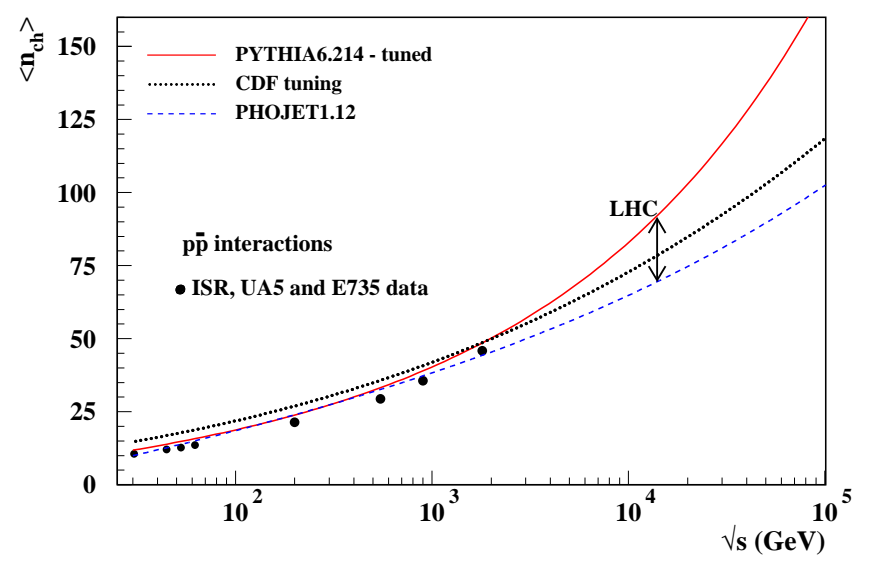

(a)

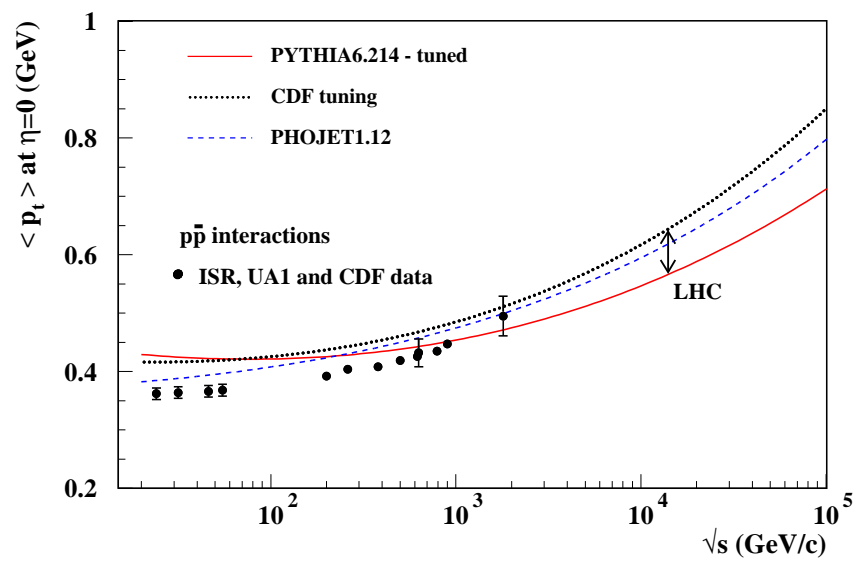

(b)

Fig. 26. (a) Average charged particle multiplicity in NSD p $\bar{p}$ collisions; (b) Average transverse momentum of charged particles $\left\langle\mathrm{p}_{t}\right\rangle$ at $\eta=0$, for NSD $\mathrm{p} \overline{\mathrm{p}}$ collisions. 
In fig. 26(b), the $\left\langle\mathrm{p}_{t}\right\rangle$ at $\eta=0$ distributions generated with the CDF tuning and PHOJET1.12 models predict higher $\left\langle\mathrm{p}_{t}\right\rangle$ than PYTHIA6.214 - tuned for nearly all $\sqrt{s}$ range. All distributions rise as $\ln ^{2}(\mathrm{~s})$ and at the LHC energy the predictions vary from $\left\langle\mathrm{p}_{t}\right\rangle=0.55 \mathrm{GeV}$ to 0.64 $\mathrm{GeV}$.

As shown in fig. 26, models which are reasonably successful in describing the wide range of data shown above, give considerably different predictions at higher energies. In the next section, the two models with best global agreement to the data, PHOJET1.12 and PYTHIA6.214 - tuned, will be used to generate minimum bias and the underlying event predictions for the LHC.

\section{LHC predictions for minimum bias and the UE}

The LHC will collide protons at centre-of-mass energies many times greater than any hadron collision ever performed in laboratory.

Models capable of reproducing the available minimum bias and underlying event data for lower colliding energies are extremely important for predicting background levels associated to many physics processes and also for understanding the complex nature of the radiation environment in which the LHC's detector systems will operate.

The study presented in previous sections indicates that the models PHOJET1.12 and PYTHIA6.214 - tuned give the best agreement to the data for both minimum bias and underlying event distributions. Here we present the LHC predictions generated by both models.

\subsection{Minimum bias distributions}

For LHC collisions (pp collisions at $\sqrt{s}=14 \mathrm{TeV}$ ) the minimum bias cross-section estimated by PYTHIA6.214 - tuned is $\sigma_{n s d}=65.7 \mathrm{mb}$ while PHOJET1.12 predicts $\sigma_{n s d}=73.8 \mathrm{mb}, 12.3 \%$ greater than the former. Hence, for the same luminosity PHOJET1.12 generates more minimum bias pp collisions than PYTHIA6.214 - tuned. We shall however, focus on the general properties per pp collision not weighted by cross-sections. The results per pp collision can later be easily scaled by the cross-section and luminosity.

Figure 27 shows charged particle density distributions in pseudorapidity for minimum bias pp collisions at $\sqrt{\mathrm{s}}=$ $14 \mathrm{TeV}$ generated by PHOJET1.12 and PYTHIA6.214 tuned. The charged particle density generated by PHOJET1.12 and PYTHIA6.214 - tuned at $\eta=0$ is 5.1 and 6.8 , respectively. In the central region $(|\eta|<2.5) \mathrm{dN}_{c h} / \mathrm{d} \eta$ is $\sim 5.5$ and $\sim 7$, respectively for PHOJET1.12 and PYTHIA6.214 - tuned. Contrasting to the agreement shown for $\mathrm{p} \overline{\mathrm{p}}$ collisions at $\sqrt{\mathrm{s}}=1.8 \mathrm{TeV}$ in fig. 21(c), at the LHC PYTHIA6.214 - tuned generates $\sim 27 \%$ more charged particle density in the central region than PHOJET1.12.

Compared to the charged particle density $\mathrm{dN}_{c h} / \mathrm{d} \eta$ measured by CDF at $1.8 \mathrm{TeV}$ (fig. 21(c) ), PYTHIA6.214

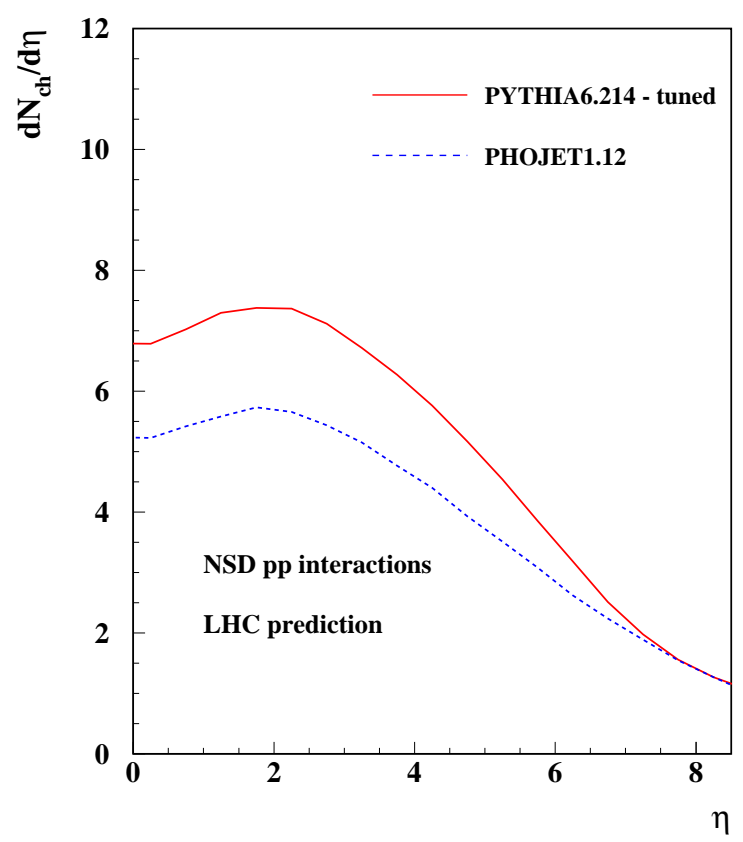

Fig. 27. Charged particle density distributions, $\mathrm{dN}_{c h} / \mathrm{d} \eta$, for NSD pp collisions at $\sqrt{\mathrm{s}}=14 \mathrm{TeV}$.Predictions generated by PYTHIA6.214 - tuned and PHOJET1.12 .

- tuned indicates a plateau rise of $\sim 70 \%$ at the LHC in the central region while PHOJET1.12 suggests a smaller rise of $\sim 35 \%$.

The average charged particle multiplicity in LHC minimum bias collisions, $\left\langle n_{c h}\right\rangle$, is 69.62 and 91.04 charged particles as predicted by PHOJET1.12 and PYTHIA6.214 - tuned, respectively. For centre-of-mass energies greater than $\sim 1 \mathrm{TeV}$, the multiple parton interaction model employed by PYTHIA and the DPM used by PHOJET lead to multiplicity distributions with different rates of increase with the energy. PYTHIA giving a steeper rise than PHOJET (figs. 21(d) and 26(a)). At $\sqrt{\mathrm{s}}=14 \mathrm{TeV}$, the activity generated in minimum bias collisions by both PHOJET1.12 and PYTHIA6.214 - tuned is $\sim 25-30 \%$ greater in the later.

The charged multiplicity distributions for LHC minimum bias events are shown in fig. 28. Predictions generated by PYTHIA6.214 - tuned and PHOJET1.12 are significantly different. At low z (low multiplicity events) PYTHIA's prediction reveals a double-peak which is not present in PHOJET's distribution. Not seen in the Tevatron charged multiplicity distribution, the double-peak in PYTHIA's prediction for the LHC reflects a difference between the string drawings for proton-proton and protonanti-proton collisions [35]. For increasingly higher $\mathrm{z}$, the shoulder structure of the distributions indicate that PYTHIA6.214 - tuned generates higher multiplicity events than PHOJET1.12, reflecting the fact that the event activity generated by multiple parton scattering in PYTHIA rises faster with the energy than the corresponding effect 


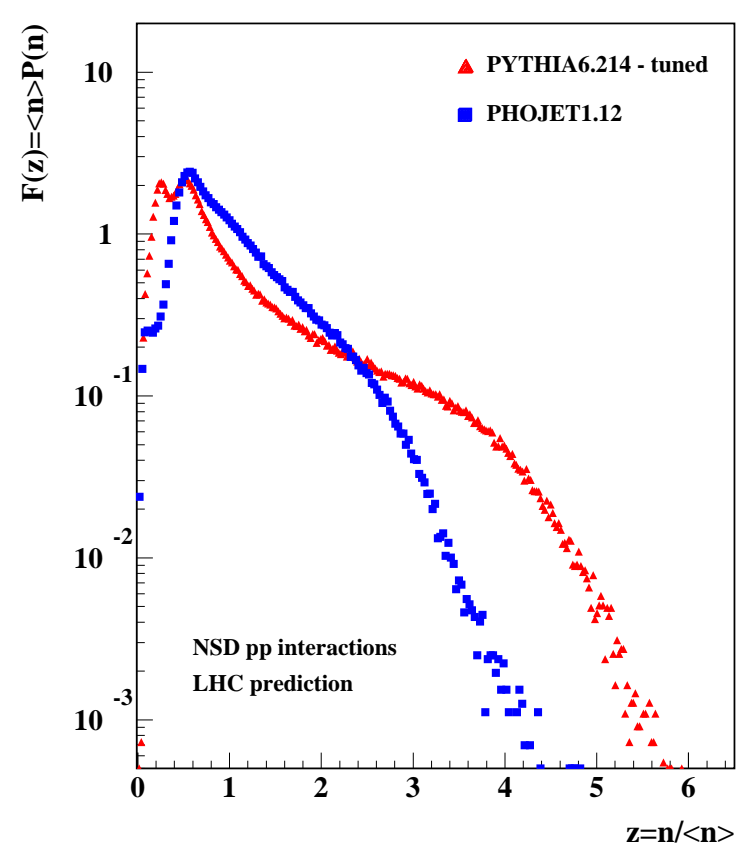

Fig. 28. Charged multiplicity distribution for NSD pp collisions at $\sqrt{\mathrm{s}}=14 \mathrm{TeV}$. Predictions generated by PYTHIA6.214 - tuned and PHOJET1.12.

due to the increase of Pomeron exchanges generated by PHOJET1.12.

A close comparison between the E735 charged multiplicity data presented in fig. 20(d) and the LHC predictions in fig. 28 shows that PHOJET1.12 does not predict a LHC charged multiplicity distribution much different of the one measured at the Tevatron, while PYTHIA6.214 tuned indicates a sizable extension of the high $\mathrm{z}$ tail of the distribution.

The $\left\langle\mathrm{p}_{t}\right\rangle$ at $\eta=0$ for charged particles in LHC minimum bias collisions predicted by PHOJET1.12 and PYTHIA6.214 - tuned models is $0.64 \mathrm{GeV}$ and $0.55 \mathrm{GeV}$, respectively. The difference of $\sim 16 \%$ in this case is proportionally smaller compared to the differences seen for particle densities in pseudorapidity and multiplicity, which are of the order of $30 \%$. Generating less particles in an average minimum bias collision at the LHC, PHOJET1.12 predicts that the average $\mathrm{p}_{t}$ per particle at $\eta=0$ is greater (or harder) than the corresponding prediction from PYTHIA6.214 - tuned.

The $\mathrm{p}_{t}$ spectrum of charged particles produced in LHC minimum bias events is displayed in fig. 29. Once again, it compares PHOJET1.12 and PYTHIA6.214 - tuned. At very low momenta, $\mathrm{p}_{t} \lesssim 0.5 \mathrm{GeV}$, the particle density predicted by PYTHIA6.214 - tuned is $\sim 40 \%$ greater than the corresponding PHOJET1.12 prediction. The difference is much smaller for higher $\mathrm{p}_{t}$, and in fact both spectra become virtually undistinguishable. The low $\mathrm{p}_{t}$ bins account for most of the multiplicity, but looking at the $\mathrm{p}_{t}$ detection capabilities at ATLAS [32] and CMS [36] for example, the detection of particles with good $\mathrm{p}_{t}$ resolution will be lim-

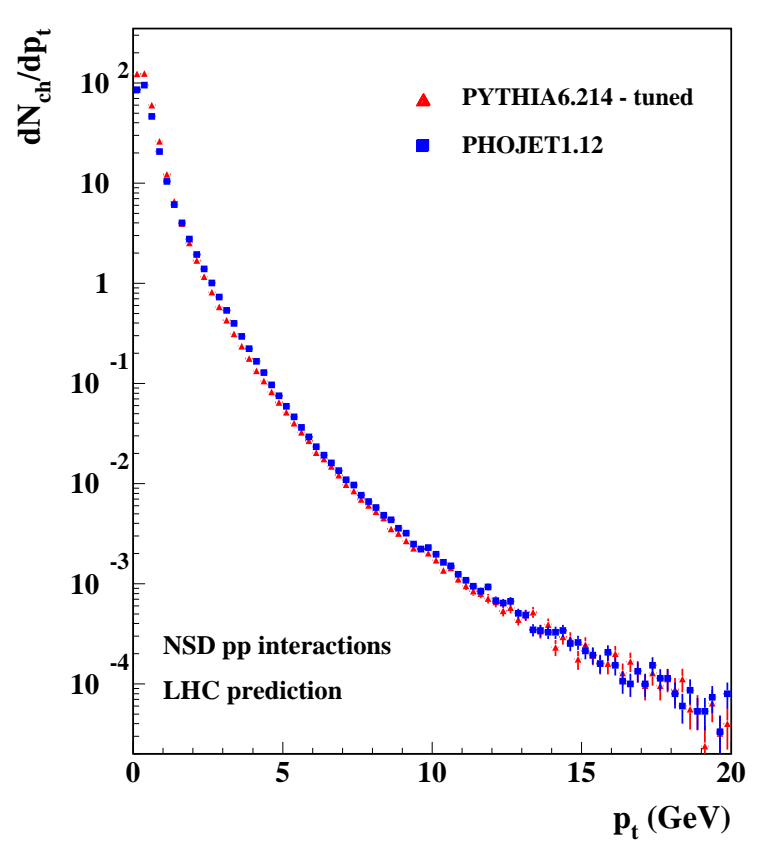

Fig. 29. Charged particle $\mathrm{p}_{t}$ spectrum for NSD pp collisions at $\sqrt{\mathrm{s}}=14 \mathrm{TeV}$.

ited to particles with $\mathrm{p}_{t}>0.5 \mathrm{GeV}[32,36]$, both models suggest very similar detected $\mathrm{p}_{t}$ spectra per pp event.

\subsection{The UE at the LHC}

Figure 30 displays PYTHIA6.214 - tuned and PHOJET1.12 predictions for the average particle multiplicity (a) and average $\mathrm{p}_{t_{\mathrm{sum}}}$ in the underlying event (b) for pp collisions at the LHC (charged particles with $p_{t}>0.5 \mathrm{GeV}$ and $|\eta|<1)$. The distributions generated by the two models are fundamentally different. Except for the events with $\mathrm{P}_{t_{1 \mathrm{jet}}} \lesssim 3 \mathrm{GeV}$, PYTHIA6.214 - tuned generates greater activity than PHOJET1.12 in both distributions shown in fig. 30 .

A close inspection of predictions for the underlying event given in fig. 30, shows that the average multiplicity in the underlying event for $\mathrm{P}_{t_{\text {ljet }}}>10 \mathrm{GeV}$ reaches a plateau at $\sim 6.5$ charged particles according to PYTHIA6.214 - tuned and $\sim 3.0$ according to PHOJET1.12. Similarly for the average $\mathrm{p}_{t_{\text {sum }}}$ in the underlying event for $\mathrm{P}_{t_{\text {ljet }}}>10 \mathrm{GeV}$, the plateaus are formed at $\sim 7.5 \mathrm{GeV}$ and $\sim 3.5 \mathrm{GeV}$ according to PYTHIA6.214 - tuned and PHOJET1.12, respectively. Compared to the underlying event distributions measured by $\mathrm{CDF}$ at $1.8 \mathrm{TeV}$ (figs. 22(a) and (b) ), PYTHIA6.214 - tuned indicates a plateau rise of $\sim 200 \%$ at the LHC while PHOJET1.12 suggests a much smaller rise of $\sim 40 \%$.

As shown in the previous section, the minimum bias predictions generated by PYTHIA6.214 - tuned and PHOJET1.12 for the central plateau of $\mathrm{dN}_{c h} / \mathrm{d} \eta$, indicate a rise of $\sim 70 \%$ and $\sim 35 \%$, respectively. These are smaller 


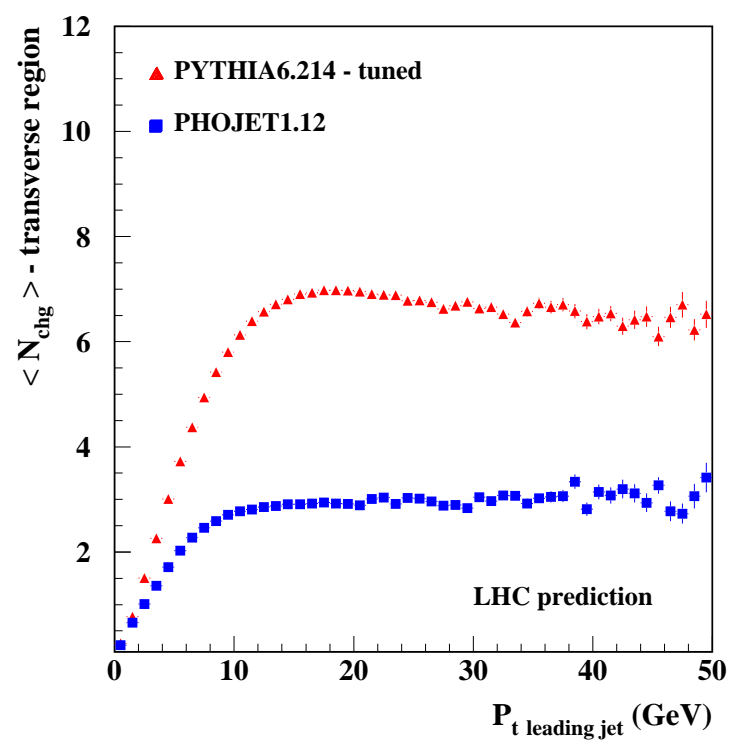

(a)

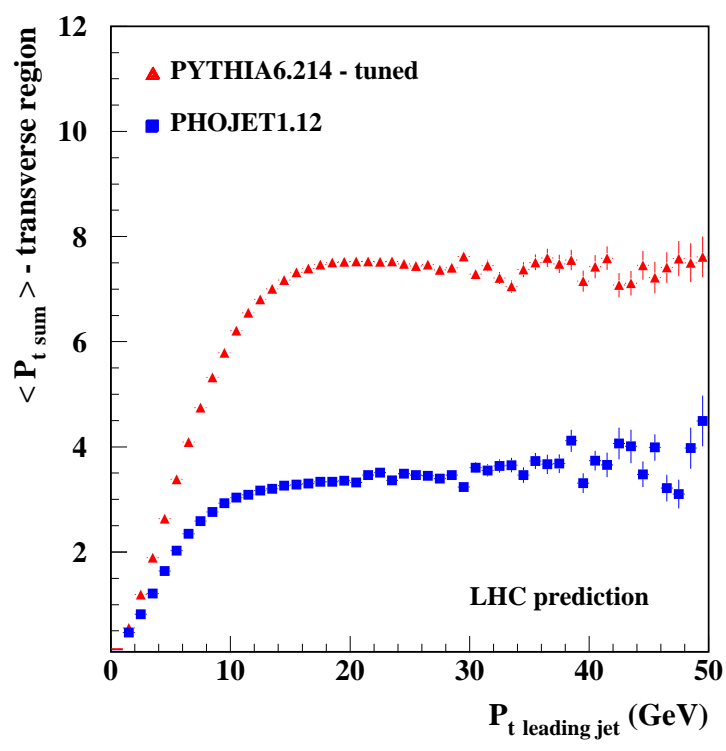

(b)

Fig. 30. PYTHIA6.214 - tuned and PHOJET1.12 predictions for: (a) average multiplicity in the underlying event and (b) average $p_{t_{\text {sum }}}$ in the underlying event.

than the predicted increase for the underlying event suggested by both models. As discussed previously, at the Tevatron, for events with $\mathrm{P}_{t_{1 \text { jet }}}>10 \mathrm{GeV}$ the particle density in the underlying event is at least a factor of two larger than the equivalent minimum bias prediction. Using similar assumptions as those adopted in the analysis for the CDF data, LHC events with $\mathrm{P}_{t_{\text {ljet }}}>10 \mathrm{GeV}$ are predicted to have a charged particle density $\mathrm{dN}_{c h} / \mathrm{d} \eta$ of $\sim 29$ charged particles per pseudorapidity unit according to PYTHIA6.214 - tuned and $\sim 13$ according to PHOJET1.12. In other words, for $\mathrm{P}_{t_{\mathrm{ljet}}}>10 \mathrm{GeV}$ the underlying event at the LHC is predicted to have a particle density $\sim 4$ times larger than its equivalent minimum bias prediction according to PYTHIA6.214 - tuned, and $\sim 2$ times larger according to PHOJET1.12.

Therefore PYTHIA6.214 - tuned predicts not only that the underlying event particle density will increase at the LHC, but it will also increase its activity compared to the equivalent minimum bias distribution. On the other hand, PHOJET1.12 estimates that the increase in charged particle density in the underlying event at the LHC will follow the same rate to the minimum bias density measured at the Tevatron. In both cases however, the underlying event density is greater than its equivalent minimum bias counterpart. Contradicting a widespread misconception that simulations involving high- $\mathrm{p}_{t}$ jets $\left(\mathrm{P}_{t_{\text {ljet }}}>10 \mathrm{GeV}\right)$ and its accompanying underlying event can be made by simply overlaying minimum bias events on top of jet events.

Further studies are currently being conducted by the CDF Collaboration aiming at a deeper understanding of the composite nature of the underlying event and minimum bias data. As indicated by the analysis shown in Ref. [37] the soft and hard components of minimum bias data behave differently with the increase of the colliding energy. The soft component of minimum bias events appears to follow the KNO scaling and has a $\mathrm{p}_{t}$ distribution at fixed multiplicity which is energy invariant. The hard minimum bias component, on the other hand, violates the KNO scaling and has a $\mathrm{p}_{t}$ distribution which rises with the energy increase [37]. Similarly, breaking down the transverse region into two regions according to the sum of particles's $\mathrm{p}_{t}$ in each of them, it has been noted that one of the regions ("transMAX") will pick up more of the hard component of the underlying event while the other region ("transMIN") will have much less activity and be populated by particles sensitive to the soft component of the underlying event, i.e. those originating from the beambeam remnant component of the underlying event [38]. The better our understanding of the soft and hard components of both minimum bias and the underlying event, the better we will be able to design models which can accurately describe and predict these processes at current collider energies and beyond.

\section{Conclusions}

Though the SM is unable to satisfactorily describe those aspects of hadron collisions which are dominated by soft partonic processes, they can be reasonably described by the MC event generators PYTHIA and PHOJET, with the appropriate tunings.

We have presented numerous comparisons between these two event generators and data for minimum bias and the underlying event. These comparisons show that PHOJET 1.12 with its default settings gives a good description of 
both sets of data, while PYTHIA6.214 needed to be tuned in order to improve its agreement to the data.

Based on the understanding of how variations in particular parameters employed by PYTHIA's multiple parton scattering model we tuned PYTHIA 6.214 to minimum bias data taken over a range of colliding energies as well as to a set of underlying event measurements made at the Tevatron.

Since we have tuned PYTHIA6.214, a new series of PYTHIA releases (version 6.3 and newer) has become available and major changes related to the description of minimum bias interactions and the underlying event have been introduced $[12,39,40]$. There is a new, more sophisticated scenario for multiple interactions, new $\mathrm{p}_{t}$-ordered initial- and final-state showers and a new treatment of beam remnants $[12,39,40]$. We are currently investigating this new model and working to obtain tunings to describe the minimum bias and underlying event data as well as to generate reliable LHC predictions [41].

The PYTHIA6.214 - tuned model and PHOJET1.12 with its default settings gave the best global statistical agreement to the data and, when compared to other proposed tunings for soft hadronic physics, they showed considerable improvements when compared to PYTHIA's default settings and to the ATLAS tuning used to produce distributions for the ATLAS-TDR. The global results obtained with PYTHIA6.214 - tuned and PHOJET1.12 are also better than those obtained with the CDF tuning. However, it is worth mentioning that the CDF proposed tuning for PYTHIA, is slightly better than our PYTHIA6.214 - tuned model and PHOJET1.12, for describing the UE measured at the Tevatron. PYTHIA6.214 - tuned has been used by the ATLAS Collaboration in the physics generation for the Atlas Data Challenge II (DC2) [42].

LHC predictions for minimum bias and the underlying event in jet events were generated with PYTHIA6.214 tuned model and PHOJET1.12. These predictions showed that although these models give comparable descriptions of lower energy data, they disagree typically by $\sim 30 \%$ in minimum bias multiplicity distributions $(\sim 16 \%$ for minimum bias $\left\langle p_{t}\right\rangle$ at $\eta=0$ ), and by a factor of $\sim 2$ for underlying event distributions. Table 10 summarises some minimum bias and underlying event related observables generated by PYTHIA6.214 - tuned and PHOJET1.12.

As shown in figures 10 and 21(d), the central plateau of charged particle density distributions, $\mathrm{dN}_{c h} / \mathrm{d} \eta$, rises with $\sqrt{\mathrm{s}}$. This happens due to the increase in the rate of semi-hard multiple parton scattering or multiple Pomeron exchanges as the colliding energy increases. PYTHIA6.214 - tuned and PHOJET1.12 generate considerably distinct LHC predictions for $\mathrm{dN}_{c h} / \mathrm{d} \eta$ at $\eta=0$ (table 10 ) which is a product of the different models employed by these two generators. PYTHIA6.214 - tuned favors a rise of the central plateau proportional to $\ln ^{2}(\mathrm{~s})$ whereas PHOJET1.12 suggests that the energy dependence of $\mathrm{dN}_{c h} / \mathrm{d} \eta$ at $\eta=0$ should be dominated by the $\ln (\mathrm{s})$ term. A fit to experimental data calculated by CDF points to a combination of $\ln ^{2}(\mathrm{~s})$ and $\ln (\mathrm{s})$ terms, albeit favoring a rise dominated by the $\ln ^{2}(\mathrm{~s})$ term [15]. Adding the LHC measurement of
$\mathrm{dN}_{c h} / \mathrm{d} \eta$ at $\eta=0$ to the historic curve which shows the $\sqrt{\mathrm{s}}$ energy evolution for the central particle density will allow us to determine the physics model that gives the best description of the data.

Compared to the Tevatron measurements, PYTHIA6.214 - tuned indicates an increase on ratio of charged particle densities in the $\mathrm{UE}\left(\mathrm{P}_{t_{1 \mathrm{jet}}}>10 \mathrm{GeV}\right)$ to its equivalent minimum bias prediction, whereas PHOJET1.12 estimates that this ratio at the LHC will remain the same as measured at the Tevatron energies. In either case, there is more activity in the UE than an average minimum bias event.

\section{Acknowledgments}

The authors wish to thank I. Skillicorn and R. St. Denis for useful suggestions. We also want to thank R. Field, T. Sjöstrand and many of our ATLAS colleagues for discussions which contributed to this work. A. M. wishes to acknowledge the support of CAPES. This work is supported by PPARC.

\section{Appendix A}

\section{Summary of PYTHIA parameters relevant to minimum bias and the underlying event}

The physical meaning of PYTHIA parameters used in this report is summarized in table 11. Detailed description of these partameters and their usage can be found in Ref. [12].

\section{Appendix B}

\section{Trigger simulation vs. MC direct selection}

Throughout this paper we have defined minimum bias events as non-single diffractive inelastic (NSD) interactions. In this appendix we check how well PYTHIA6.214 - tuned (table 4) and PHOJET1.12 describe NSD interaction by direct selection $(\operatorname{MSUB}(94)=1$ and $\operatorname{MSUB}(95)=1$ in PYTHIA6.214, and processes $\operatorname{IPRON}(1,1), \operatorname{IPRON}(4,1)$ and IPRON $(7,1)$ switched on in PHOJET1.12) when compared to trigger simulations which reproduce the selection criteria applied by UA5 [14] and CDF [15].

\section{B1 - UA5 trigger system}

The UA5 detector was optimised for the study of charged particle multiplicity distributions for NSD interactions. Two systems of scintillator counter hodoscopes were used to select NSD events. As schematically shown in fig. 31(a), the trigger hodoscopes $\mathrm{H} 1$ and $\mathrm{H} 2$ were positioned 4.6 $\mathrm{m}$ away from the interaction point following the beampipe direction covering the region $2.0<|\eta|<3.9$, while 


\begin{tabular}{|c|c|c|c|}
\hline Observable & $\begin{array}{c}\text { PYTHIA6.214 - } \\
\text { tuned }\end{array}$ & PHOJET1.12 & $\Delta \%=\frac{\left(X_{\text {high }}-Y_{\text {low }}\right) \times 100}{Y_{\text {low }}}$ \\
\hline$\overline{\sigma_{t o t}(\mathrm{mb})}$ & 101.5 & 119.1 & 17.3 \\
\hline$\sigma_{\text {elas }}(\mathrm{mb})$ & 22.5 & 34.5 & 53.3 \\
\hline$\sigma_{n s d}(\mathrm{mb})$ & 65.7 & 73.8 & 12.3 \\
\hline \multicolumn{4}{|c|}{ Minimum bias Predictions } \\
\hline$\left\langle n_{c h}\right\rangle$ & 91.0 & 69.6 & 30.7 \\
\hline $\begin{array}{l}d N_{c h} / d \eta \text { plateau } \\
\quad \text { for }|\eta|<2.5\end{array}$ & $\sim 7.0$ & $\sim 5.5$ & 27.3 \\
\hline$d N_{c h} / d \eta$ at $\eta=0$ & 6.8 & 5.1 & 33.3 \\
\hline$\left\langle p_{t}\right\rangle$ at $\eta=0(\mathrm{GeV})$ & 0.55 & 0.64 & 16.4 \\
\hline $\begin{array}{c}\mathrm{n}_{\text {tot }}(|\eta|<15) \\
\quad \text { per pp event }\end{array}$ & 158.4 & 115.1 & 37.6 \\
\hline $\mathrm{n}_{\text {tot }}(|\eta|<2.5)$ & 60.9 & 45.5 & 33.8 \\
\hline \multicolumn{4}{|c|}{ Underlying Event Predictions (approx. values) } \\
\hline $\begin{array}{c}\left\langle N_{\text {chg }}\right\rangle \\
\mathrm{P}_{t_{1 \mathrm{jet}}}>10 \mathrm{GeV}\end{array}$ & 6.5 & 3.0 & $\sim 115$ \\
\hline $\mathrm{P}_{t_{t_{\text {jet }}}}\left\langle\mathrm{p}_{t_{\text {sum }}}\right\rangle \mathrm{GeV}$ & 7.5 & 3.5 & $\sim 115$ \\
\hline $\begin{array}{c}d N_{c h} / d \eta \text { for } \\
\mathrm{P}_{t_{1 \text { jet }}}>10 \mathrm{GeV}\end{array}$ & 29.0 & 13.3 & $\sim 120$ \\
\hline $\begin{array}{c}\text { UE/Min-bias } \\
\mathrm{P}_{t_{1 \mathrm{jet}}}>10 \mathrm{GeV}\end{array}$ & 4 & 2 & 100 \\
\hline
\end{tabular}

Table 10. PYTHIA6.214 - tuned and PHOJET1.12 results for pp collisions event generation at $\sqrt{s}=14$ TeV.

the forward hodoscopes F1 and F2 were positioned at $5.0 \mathrm{~m}$ from the interaction point, covering the $\eta$ range $3.6<|\eta|<5.6[14]$. NSD events were selected by requiring a coincidence between a beam crossing and at least one hit in each of the trigger systems, which covered the $\eta$ ranges $-5.6<\eta<-2.0$ and $2.0<\eta<5.6$, respectively.

The UA5 minimum bias trigger excluded most of the single-diffractive events and recorded about 95\% of the NSD events [19]. Off-line software cuts were also used to eliminate background [14].

\section{B2 - CDF minimum bias trigger}

During Tevatron's run I, the CDF detector used a system of beam-beam counters (BBC) to trigger the detector on NSD p $\bar{p}$ interactions [15]. The BBC consisted of two sets of scintillation counters placed along the beam axis $\sim 5.9$ $\mathrm{m}$ on either side of the interaction point. The $\eta$ range covered by the beam-beam counters was $3.2<|\eta|<5.9$. The vertex time-projection chamber (VTPC) was used to measure the trajectory of charged particles as they exited the beam pipe and also to reconstruct the $\mathrm{p} \overline{\mathrm{p}}$ interaction point. A cross-sectional representation of one quadrant of CDF's inner detector, including the BBC and VTPC, is shown in fig. 31(b).

CDF selected NSD events by requiring at least one hit in each set of the $\mathrm{BBC}$ counters in coincidence with the

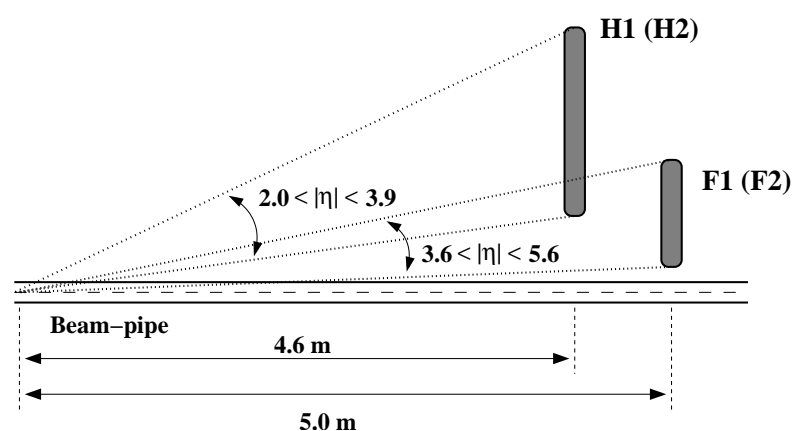

(a)

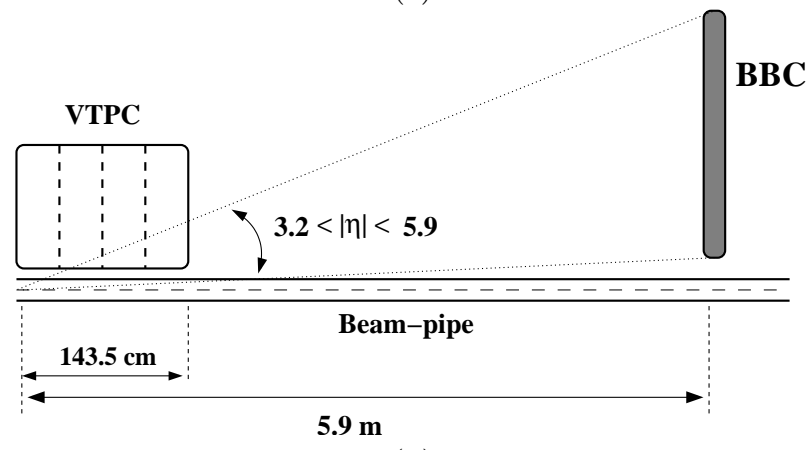

(b)

Fig. 31. Cross-sectional representation of one quadrant of (a) UA5 and (b) CDF, showing the main triggering elements used to select minimum bias events. 


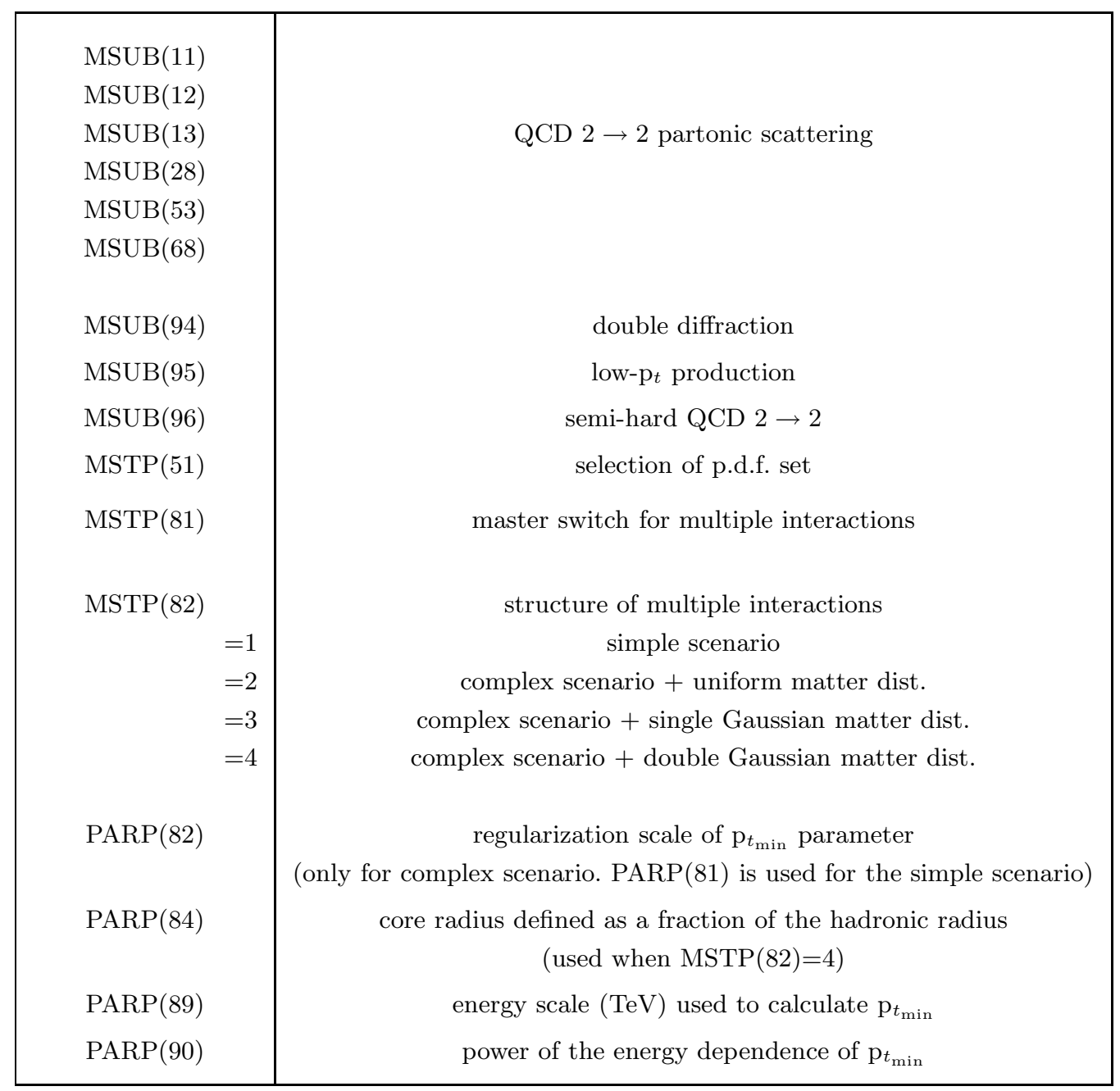

Table 11. Physical meaning summary of PYTHIA parameters used in this study.

beam crossing. As for the UA5 data, several off-line software cuts were also applied by the CDF Collaboration in order to eliminate beam-gas background and select purer samples of minimum bias $\mathrm{p} \overline{\mathrm{p}}$ collisions [15].

\section{B3 - Trigger simulation vs. direct selection}

Trigger simulations were performed by generating all possible processes which take place in a typical high-energy hadron collisions and then applying the same triggering requirements used to select NSD events by UA5 for $p \bar{p}$ collisions at $\sqrt{s}=200,546$ and $900 \mathrm{GeV}$, and by CDF for $\mathrm{p} \overline{\mathrm{p}}$ collisions at $\sqrt{s}=1.8 \mathrm{TeV}$. Thus, the initial sample of generated collisions included elastic and all classes of inelastic processes (single and double diffraction and nondiffractive inelastic) and after being filtered by the trigger selection, only minimum bias events remained. These simulations were done using both PYTHIA6.214 - tuned model and PHOJET1.12 with its default parameters.

The results obtained with the trigger simulations at various energies were compared to those generated by set- ting the MC programs to generate exclusively minimum bias events, i.e., setting $\operatorname{MSUB}(94)=1$ and $\operatorname{MSUB}(95)=1$ in PYTHIA6.214, and switching on the processes IPRON$(1,1), \operatorname{IPRON}(4,1)$ and $\operatorname{IPRON}(7,1)$ in PHOJET1.12 . Table 12 shows the minimum bias cross-sections obtained by simulating the trigger selection as applied by UA5 and $\mathrm{CDF}$, compared to the cross-sections obtained by the direct selection of only NSD interaction.

As already discussed in previous sections, there is a difference in the NSD inelastic cross-section predicted by PYTHIA and PHOJET. However, for both event generators, the differences between cross-sections generated by the two methods (i.e. trigger simulation and direct selection of processes) is typically less than $5 \%$.

Figure 32 displays charged particle density distributions, $\mathrm{dN}_{c h} / \mathrm{d} \eta$, for NSD $\mathrm{p} \overline{\mathrm{p}}$ collisions, comparing results obtained using the trigger simulation to those from the direct selection of processes for both PYTHIA6.214 - tuned and PHOJET1.12 . No significant differences are observed between the two simulations at $\sqrt{\mathrm{s}}=900 \mathrm{GeV}$ or $\sqrt{\mathrm{s}}=1.8$ $\mathrm{TeV}$. The $\chi^{2} /$ d.o.f. obtained with PYTHIA6.214 - tuned using the trigger simulation are $\chi^{2} / 19$ d.o.f. $=1.3$ and 


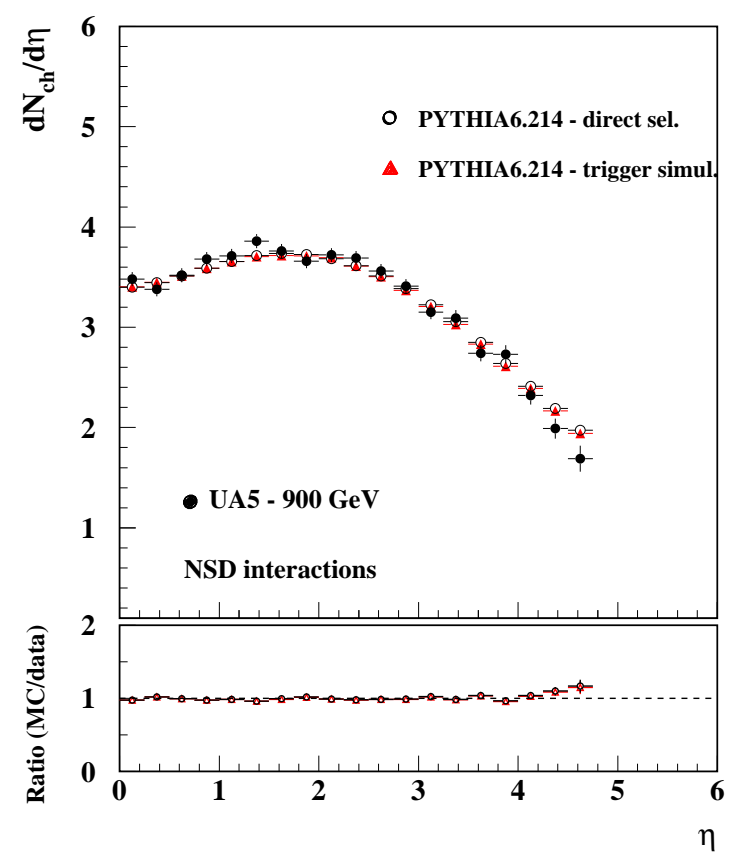

(a)

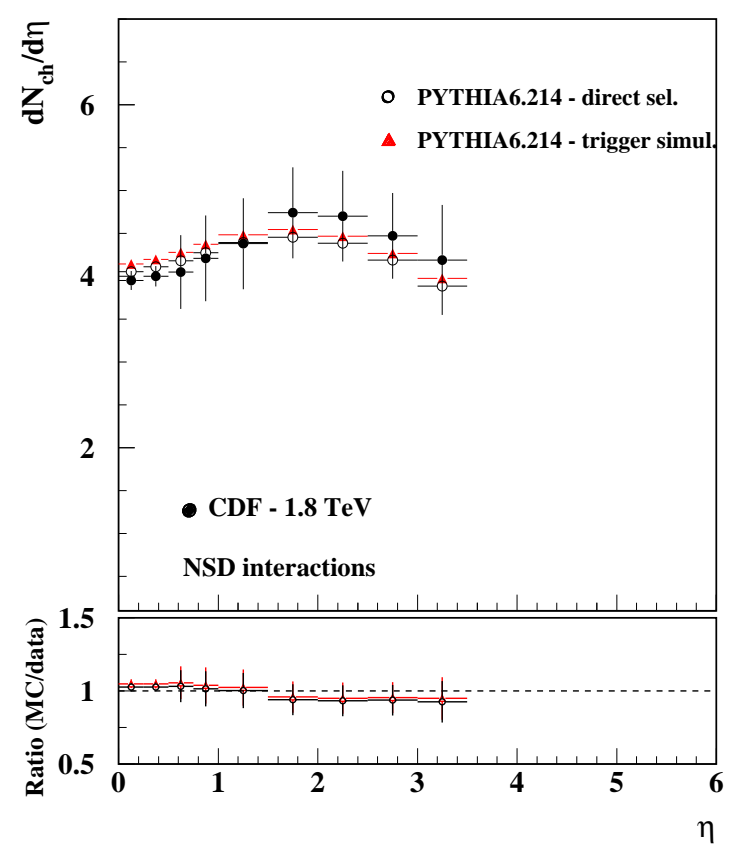

(c)

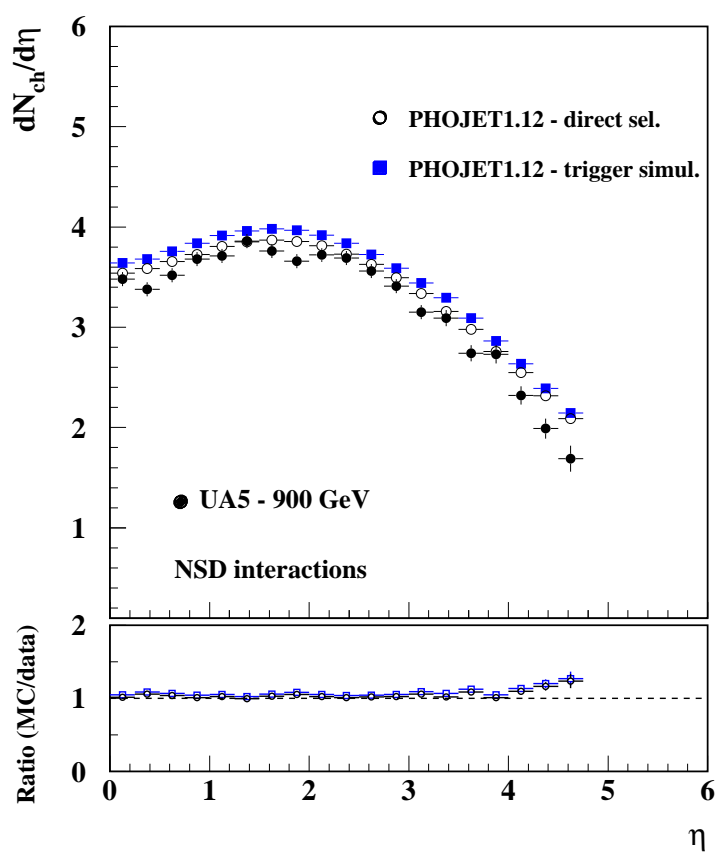

(b)

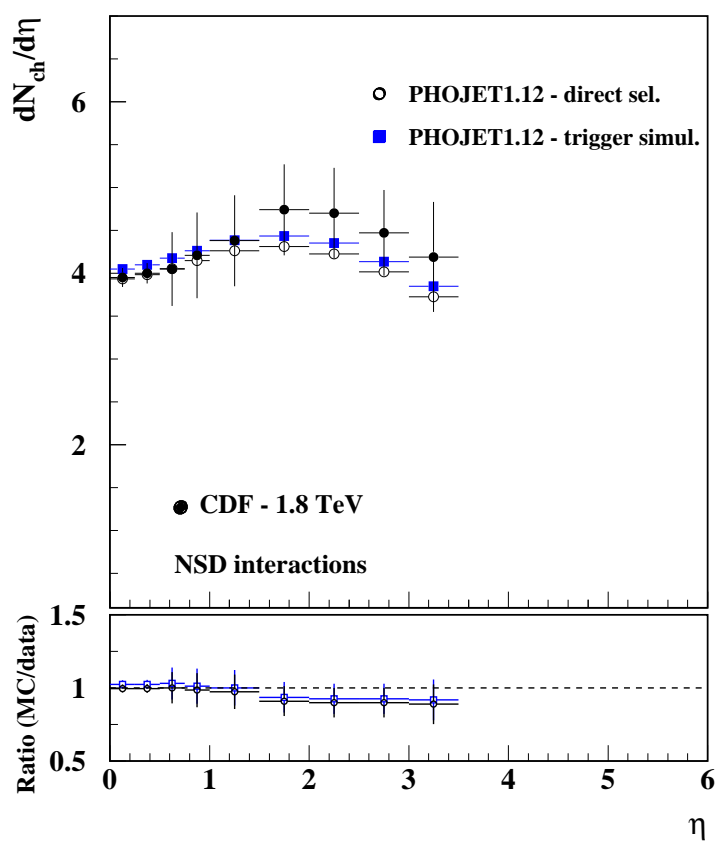

(d)

Fig. 32. Charged particle density distributions, $\mathrm{dN}_{c h} / \mathrm{d} \eta$, for NSD $\mathrm{p} \overline{\mathrm{p}}$ collisions comparing results obtained using the trigger simulation to those from the direct selection of processes with (a) PYTHIA6.214 - tuned and (b) PHOJET1.12 at $\sqrt{\mathrm{s}}=900$ $\mathrm{GeV}$, and (c) PYTHIA6.214 - tuned and (d) PHOJET1.12 at $\sqrt{\mathrm{s}}=1.8 \mathrm{TeV}$. 


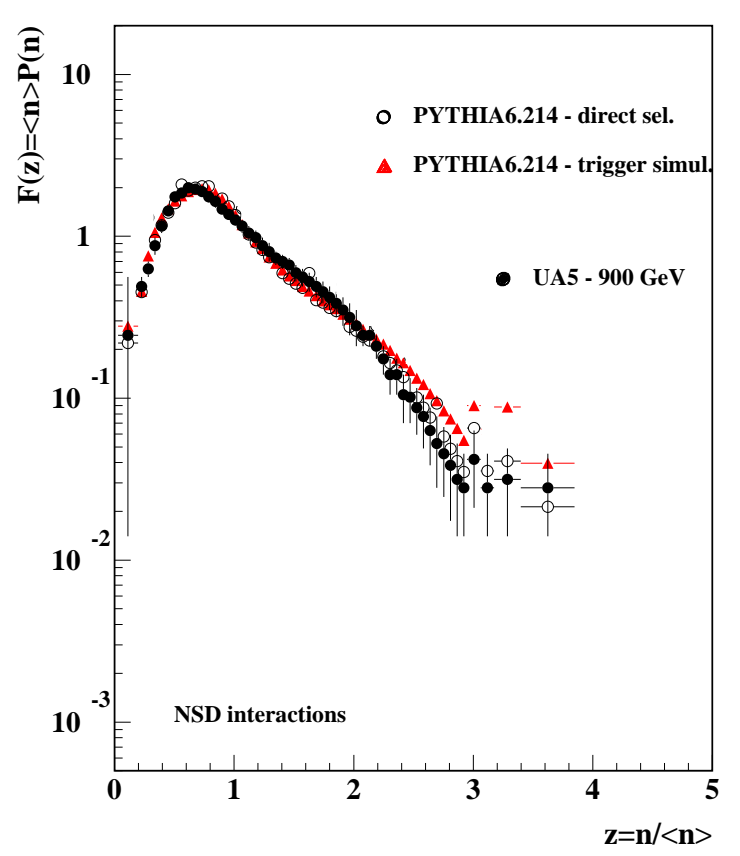

(a)

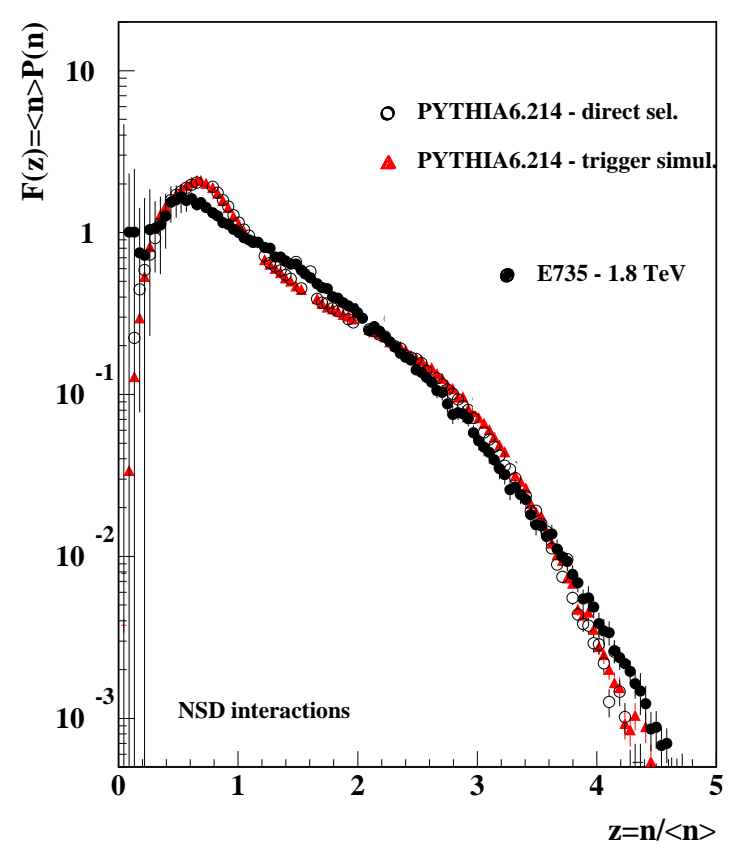

(c)

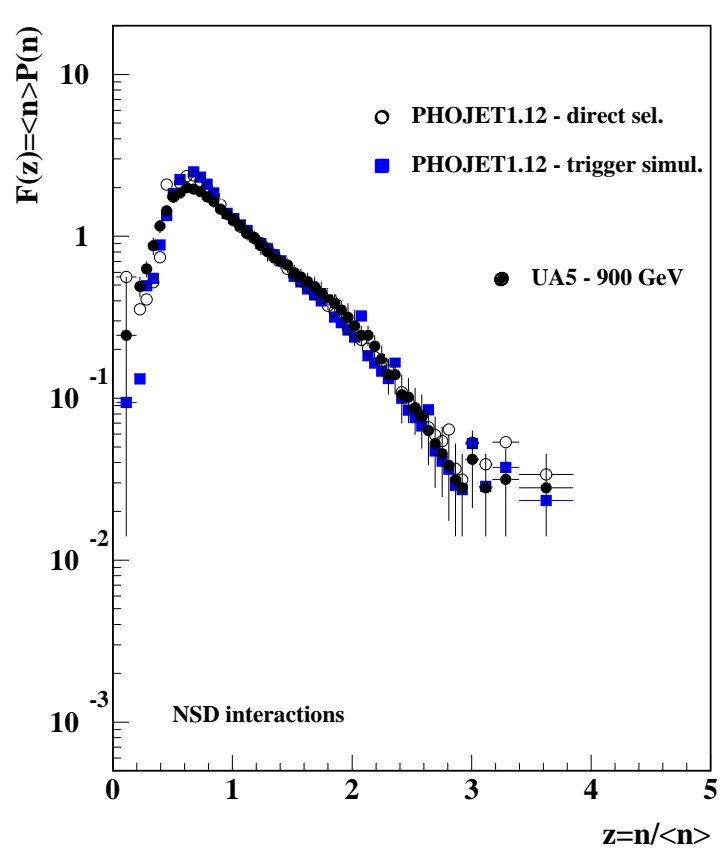

(b)

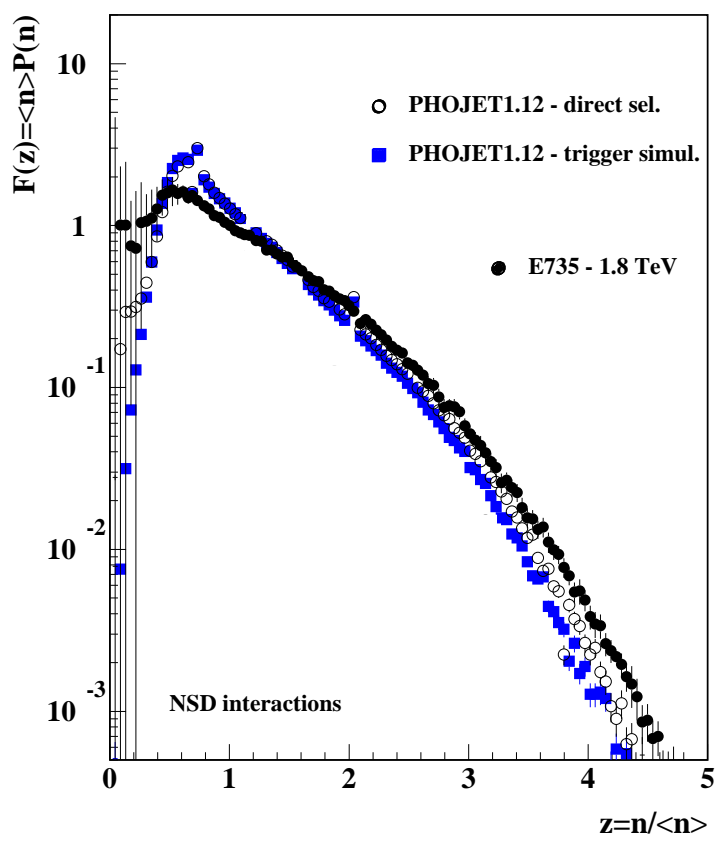

(d)

Fig. 33. Charged multiplicity distributions for NSD $\mathrm{p} \overline{\mathrm{p}}$ collisions comparing results obtained using the trigger simulation to those from the direct selection of processes with (a) PYTHIA6.214 - tuned and (b) PHOJET1.12 at $\sqrt{\mathrm{s}}=900 \mathrm{GeV}$, and (c) PYTHIA6.214 - tuned and (d) PHOJET1.12 at $\sqrt{\mathrm{s}}=1.8 \mathrm{TeV}$. 


\begin{tabular}{|c|c|c|}
\hline & $\begin{array}{c}\text { PYTHIA6.214 - } \\
\text { tuned }\end{array}$ & PHOJET1.12 \\
\hline \multicolumn{3}{|l|}{$200 \mathrm{GeV}$} \\
\hline Trigger Simul. & $32.8 \mathrm{mb}$ & $34.3 \mathrm{mb}$ \\
\hline$M C$ direct select. & $32.4 \mathrm{mb}$ & $33.4 \mathrm{mb}$ \\
\hline \multicolumn{3}{|l|}{$\mathrm{p} \overline{\mathrm{p}}$ at $\sqrt{s}=546 \mathrm{GeV}$} \\
\hline Trigger Simul. & $38.2 \mathrm{mb}$ & $38.2 \mathrm{mb}$ \\
\hline$M C$ direct select. & $37.7 \mathrm{mb}$ & $39.9 \mathrm{mb}$ \\
\hline \multicolumn{3}{|l|}{$\mathrm{p} \overline{\mathrm{p}}$ at $\sqrt{s}=900 \mathrm{GeV}$} \\
\hline Trigger Simul. & $41.4 \mathrm{mb}$ & $43.4 \mathrm{mb}$ \\
\hline$M C$ direct select. & $40.8 \mathrm{mb}$ & $44.6 \mathrm{mb}$ \\
\hline \multicolumn{3}{|l|}{$\mathrm{p} \overline{\mathrm{p}}$ at $\sqrt{s}=1.8 \mathrm{TeV}$} \\
\hline Trigger Simul. & $44.3 \mathrm{mb}$ & $51.4 \mathrm{mb}$ \\
\hline$M C$ direct select. & $45.8 \mathrm{mb}$ & $52.2 \mathrm{mb}$ \\
\hline
\end{tabular}

Table 12. Comparison of NSD cross-sections generated using trigger simulation and direct selection.

$\chi^{2} / 9$ d.o.f. $=0.8$ for $\mathrm{dN}_{c h} / \mathrm{d} \eta$ comparisons at $\sqrt{\mathrm{s}}=900$ $\mathrm{GeV}$ and $\sqrt{\mathrm{s}}=1.8 \mathrm{TeV}$. The corresponding $\chi^{2}$ results obtained with PYTHIA6.214 - tuned using the direct selection of processes are $\chi^{2} / 19$ d.o.f. $=1.4$ and $\chi^{2} / 9$ d.o.f. $=$ 0.3 for $\mathrm{dN}_{c h} / \mathrm{d} \eta$ comparisons at $\sqrt{\mathrm{s}}=900 \mathrm{GeV}$ and $\sqrt{\mathrm{s}}=$ $1.8 \mathrm{TeV}$. For PHOJET1.12, the results are $\chi^{2} / 19$ d.o.f. $=$ 9.9 (3.8) and $\chi^{2} / 9$ d.o.f. $=0.4(0.3)$ for $\mathrm{dN}_{c h} / \mathrm{d} \eta$ comparisons with the trigger (direct selection) simulation at $\sqrt{\mathrm{s}}$ $=900 \mathrm{GeV}$ and $\sqrt{\mathrm{s}}=1.8 \mathrm{TeV}$.

Figure 33 shows charged multiplicity distributions for NSD p $\overline{\mathrm{p}}$ collisions at $\sqrt{\mathrm{s}}=900 \mathrm{GeV}$ and $\sqrt{\mathrm{s}}=1.8 \mathrm{TeV}$ comparing, once again, results obtained using the trigger simulation to those from the direct selection of processes. Similarly to comparisons of reconstructed NSD cross-sections (table 12) and $\mathrm{dN}_{c h} / \mathrm{d} \eta$ distributions (fig. 32), the charged multiplicity distributions generated by the different methods agree reasonably well. The only noticeable difference is observed for the low-z region of the charged multiplicity distributions, especially at $\sqrt{\mathrm{s}}=1.8 \mathrm{TeV}$. Distributions generated by the trigger simulation display less low-multiplicity events than those generated with the direct selection of processes. This is probably due to the fact that the trigger requirements cause the rejection of some double-diffractive events (typically low-multiplicity or low-z events) in addition to the single-diffractive interactions.

For the charged multiplicity distributions shown in fig. 33 the $\chi^{2} /$ d.o.f. obtained with PYTHIA6.214 - tuned using the trigger simulation are $\chi^{2} / 54$ d.o.f. $=1.6$ and $\chi^{2} / 128$ d.o.f. $=15.2$ for charged multiplicity distribution comparisons at $\sqrt{\mathrm{s}}=900 \mathrm{GeV}$ and $\sqrt{\mathrm{s}}=1.8 \mathrm{TeV}$. The corresponding $\chi^{2}$ results obtained with PYTHIA6.214 tuned using the direct selection of processes are $\chi^{2} / 54$ d.o.f. $=6.3$ and $\chi^{2} / 128$ d.o.f. $=12.5$ for charged multiplicity distribution comparisons at $\sqrt{\mathrm{s}}=900 \mathrm{GeV}$ and $\sqrt{\mathrm{s}}=1.8$ TeV. For PHOJET1.12, the results are $\chi^{2} / 54$ d.o.f. $=6.4$ (3.4) and $\chi^{2} / 128$ d.o.f. $=12.9(10.6)$ for charged mul- tiplicity distribution comparisons with the trigger (direct selection) simulation at $\sqrt{\mathrm{s}}=900 \mathrm{GeV}$ and $\sqrt{\mathrm{s}}=1.8 \mathrm{TeV}$.

The results for $\chi^{2} /$ d.o.f. obtained in comparisons shown in figs. 32 and 33 indicate that using the direct selection of processes for minimum bias events, the agreement between MC distributions and data is comparable to the results which used a trigger simulation. The overall $\chi^{2} /$ d.o.f. obtained by combining the distributions in both figures, is $\chi^{2} / 210$ d.o.f. $=9.8$ for PYTHIA6.214 - tuned using the trigger simulation and $\chi^{2} / 210$ d.o.f. $=9.3$ for PYTHIA6.214 - tuned using the direct selection of processes. For PHOJET1.12, the results are $\chi^{2} / 210$ d.o.f. $=10.5$ and 7.7 for the trigger and direct selection simulation, respectively.

Based on the comparisons shown above, we verify that the direct selection of NSD interactions in both PYTHIA 6.214 and PHOJET1.12 agrees reasonably well to the crosssections and minimum bias distributions predicted by the same event generators with the trigger simulation. 


\section{References}

1. The Large Hadron Collider, Conceptual Design, CERNAC-95-05 LHC, October 1995.

2. P. D. B. Collins and A. D. Martin, Rep. Prog. Phys. 45 (1982) 335;

P. D. B. Collins and A. D. Martin, Hadron Interactions (Graduate student series in physics), Adam Hilger, Bristol, 1984.

3. M. E. Peskin and D. V. Schroeder, An Introduction to Quantum Field Theory, Perseus Books, 1995;

F. Mandl and G. Shaw, Quantum Field Theory, Wiley, 1993

F. Halzen and A. Martin, Quarks and Leptons: An Introductory Course in Modern Particle Physics, Wiley, 1984.

4. I. Borjanovic et al., Eur. Phys. J., C 39 (2005) s63.

5. S. Asai et al., Eur. Phys. J., C 32 (2004) s19.

6. I. Dawson et al., Nucl. Instr. and Meth. A 510 (2003) 219.

7. T. Akesson et al., Z. Phys. C 34 (1987) 163.

8. J. Alitti et al., Phys. Lett. B 268 (1991) 145.

9. F. Abe et al., Phys. Rev. D 47 (1993) 4857;

F. Abe et al., Phys. Rev. Lett. 79 (1997) 584;

F. Abe et al., Phys. Rev. D 56 (1997) 3811.

10. A. Capella et al., Phys. Lett. 81B(1) (1979) 68;

A. Capella and J. T. T. Van, Phys. Lett. 93B(1,2) (1980) 146 ;

A. Capella and J. T. T. Van, Z. Phys. C 10 (1981) 249;

A. Capella et al. Phys. Rep. 236(4,5) (1994) 225.

11. T. Sjöstrand and M. van Zijl, Phys. Rev. D 36(7) (1987) 2019.

12. T. Sjöstrand, Comput. Phys. Commun. 82 (1994) 74; T. Sjöstrand et al., Comput. Phys. Commun. 135 (2001) 238;

T. Sjöstrand, L. Lönnblad and S. Mrenna PYTHIA 6.2 Physics and Manual, hep-ph/0108264, August 2001;

Public web-pages at: http://www.thep.lu.se/ torbjorn/Pythia.html .

13. A. Breakstone et al., Phys. Rev. D 30(3) (1984) 528.

14. G. J. Alner et al., Phys. Rep. 154(5,6) (1987) 247.

15. F. Abe et al., Phys. Rev. D 41(7) (1990) 2330.

16. T. Alexopoulos et al., Phys. Lett. B 435 (1998) 453; S. G. Matinyan and W. D. Walker, Phys. Rev. D. 59 (1999) 034022

17. Z. Koba, H. B. Nielsen and P. Olesen, Nucl. Phys. B 40 (1972) 317.

18. G. J. Alner et al., Phys. Lett. 167B (1986) 476.

19. R. E. Ansorge et al., Z. Phys. C 43 (1989) 357.

20. T. Affolder et al., Phys. Rev. D 65 (2002) 092002.

21. J. M. Butterworth and M. H. Seymour, JIMMY4: Multiparton Interactions in Herwig for the LHC, October 2004. http://jetweb.hep.ucl.ac.uk/JIMMY/index.html .

22. R. Engel, Z. Phys. C 66 (1995) 203;

R. Engel, PHOJET manual (program version $1.05 \mathrm{c}$, June 96) http://physik.uni-leipzig.de/ eng/phojet.html .

23. J. Dischler and T Sjöstrand, EPJdirect C2 (2001) 1.

24. A. Donnachie and P. V. Landshorff, Phys. Lett. B 296 (1992) 227.

25. K. Hahn and J. Ranft, Phys. Rev. D 41(5) (1990) 1463.

26. J. R. Forshaw and D. A. Ross, Quantum Chromodynamics and Pomeron, Cambridge (1997).

27. B. Andersson et al., Phys. Rep. 97(1983) 31.

28. G. A. Schuler and T. Sjöstrand, Phys. Rev. D 49(5) (1994) 2257.
29. K. Igi and M. Ishida, Phys. Lett. B 622(2005) 286.

30. K. Hagiwara et al., Phys. Rev. D 66, 010001 (2002).

31. G. Cowan, Statistical Data Analysis, Oxford, 1998.

32. ATLAS Detector and Physics Performance, Technical Design Report, CERN/LHCC/99-14, May 1999;

ATLAS Technical Proposal, Radiation environment, CERN/LHCC/94-43, December 1994.

33. A Kupčo, $M C$ event generators and their predictions for minimum bias events at LHC energies, ATL-PHYS-99-019, November 1999.

34. R. Field, Min-Bias and the Underlying Event at the Tevatron and the $L H C$, talk presented at the Fermilab ME/MC Tuning Workshop, Fermilab, 4th October 2002.

35. T. Sjöstrand, private communication.

36. CMS Collaboration, Technical Proposal, CERN/LHCC/94-38 (1994).

37. D. Acosta et al., Phys. Rev. D 65 (2002) 072005.

38. R. Field, The Underlying Event in Hard Scattering Processes, CDF/MIN-BIAS/PUBLIC/ 5746, contribution to Snowmass 2001.

39. T. Sjöstrand and P. Z. Skands, JHEP 03 (2004) 053.

40. T. Sjöstrand and P. Z. Skands, Eur. Phys. J. C 39 (2005) 129.

41. C. Buttar et al., The Underlying Event. In: 'HERA and the LHC: A Workshop on the Implications of HERA for LHC Physics' (Proceedings), 26 Mar 2004 - 24 Mar 2005, CERN Geneva Switzerland and DESY Hamburg Germany - A. de Roeck (ed.); H. Jung (ed.) . CERN-2005-014, CERN Document (Proceedings) December 2005;

A. Moraes and C. Buttar, Tuning models for minimum bias and the underlying event. In: 'Physics at Tev Colliders 2005' QCD, EW and Higgs Working Group: Summary Report, (2006) hep-ph/0604120.

42. Physics Generation for Atlas Data Challenges (ATLASDC): http://www-theory.lbl.gov/\%7Eianh /dc/dc2.html . 